\title{
EMC/EMI Characterization using a Novel 3D System on Package
}

\author{
Bachir Tamer, B.Eng. \\ A thesis submitted to the Faculty of Graduate Studies and \\ Research in partial fulfillment of the requirements for the degree of \\ Master of Applied Science \\ In \\ Electrical Engineering
}

Ottawa-Carleton Institute for Electrical Engineering

Department of Electronics, Carleton University

Ottawa, Canada

June 2009

(C) copyright

2009, Bachir Tamer 


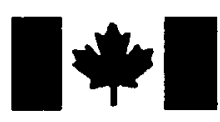

\author{
Library and Archives \\ Canada \\ Published Heritage \\ Branch \\ 395 Wellington Street \\ Ottawa ON K1A ON4 \\ Canada
}

Bibliothèque et

Archives Canada

Direction du

Patrimoine de l'édition

395 , rue Wellington

Ottawa ON K1A ON4

Canada
Your file Votre référence
ISBN: $978-0-494-60233-1$
Our file Notre référence
ISBN: $978-0-494-60233-1$
NOTICE:

The author has granted a nonexclusive license allowing Library and Archives Canada to reproduce, publish, archive, preserve, conserve, communicate to the public by telecommunication or on the Internet, loan, distribute and sell theses worldwide, for commercial or noncommercial purposes, in microform, paper, electronic and/or any other formats.

The author retains copyright ownership and moral rights in this thesis. Neither the thesis nor substantial extracts from it may be printed or otherwise reproduced without the author's permission.
AVIS:

L'auteur a accordé une licence non exclusive permettant à la Bibliothèque et Archives Canada de reproduire, publier, archiver, sauvegarder, conserver, transmettre au public par télécommunication ou par l'Internet, prêter, distribuer et vendre des thèses partout dans le monde, à des fins commerciales ou autres, sur support microforme, papier, électronique et/ou autres formats.

L'auteur conserve la propriété du droit d'auteur et des droits moraux qui protège cette thèse. $\mathrm{Ni}$ la thèse ni des extraits substantiels de celle-ci ne doivent être imprimés ou autrement reproduits sans son autorisation.
In compliance with the Canadian Privacy Act some supporting forms may have been removed from this thesis.

While these forms may be included in the document page count, their removal does not represent any loss of content from the thesis.
Conformément à la loi canadienne sur la protection de la vie privée, quelques formulaires secondaires ont été enlevés de cette thèse.

Bien que ces formulaires aient inclus dans la pagination, il n'y aura aucun contenu manquant.

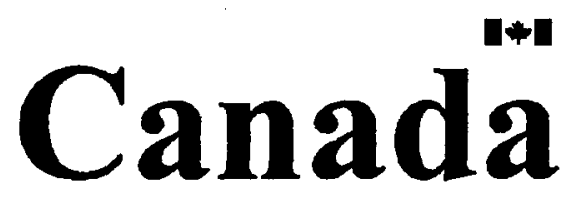




\section{Abstract}

A low temperature co-fired ceramic (LTCC) module for the near field scan of magnetic fields is presented for the first time. The module comprises 64 miniature antennas, and 9 multiplexer chips all fully integrated. The design is aimed for rapid characterization of electromagnetic compatibility (EMC) and specifically for electromagnetic interference (EMI). The multiplexer chips are all similar and they are built using the CMOS $0.13 \mu \mathrm{m}$ technology from IBM through CMC. The chip occupies a space of $0.5 \mathrm{~mm} 2$. The power consumption of the multiplexer chip, when in operation, is of $3.5 \mathrm{~mW}$. Each miniature antenna is formed of a semi-rectangular loop in all 3 planes $X Y, X Z$, and $Y Z$. The longest dimension in each plane is of $1 \mathrm{~mm}$, and the separation between the antenna elements is $1 \mathrm{~mm}$ also. The proposed system on package (SoP) serves as a tool for engineers for fast characterization of EMI on their fabricated chips or printed circuit boards (PCB). 


\title{
Acknowledgements
}

\author{
I thank God for blessing me
}

I thank Mom and Dad for being proud and encouraging me I thank my Ottawa family, uncles Issa and Mike, aunts Georgette and Mary, and families

I thank Dr. Langis Roy for his support

I thank Pietro, Dritan, and Steve for having coffee with me every morning

I thank Blazenka, Peggy, Sylvie for being helpful and funny I thank Nagui for all his help

I thank Yasser for the encouragement

I thank Barbora, Dan, Svetlana, Adam, Dritan for being great officemates

I thank Atif, Greg, Arsalan, Tosin, for the boring / funny at times Wednesday meetings

I thank OCE for allowing me to spend 6 weeks at INSA de Toulouse I thank the LATTIS people at INSA de Toulouse for being generous and supportive I thank Reza for the interesting lunch topics 


\section{Table of Contents}

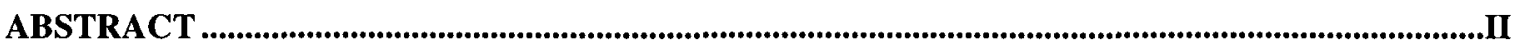

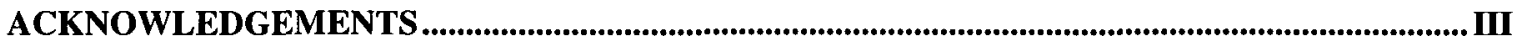

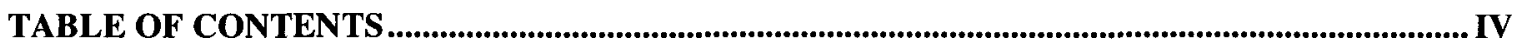

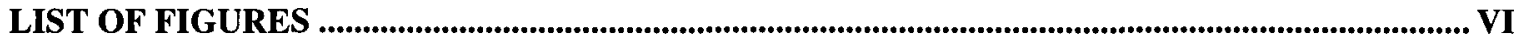

LIST OF ABBREVIATIONS AND SYMBOLS ................................................................................ VIII

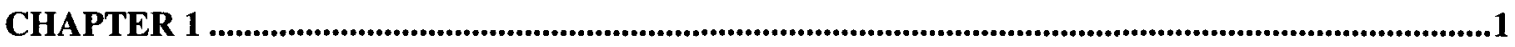

INTRODUCTION

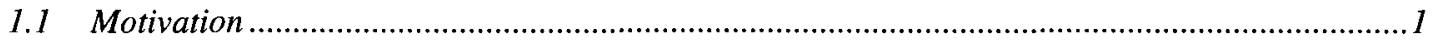

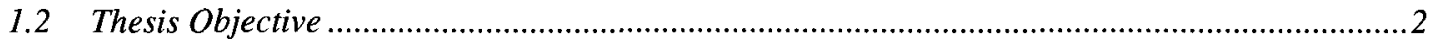

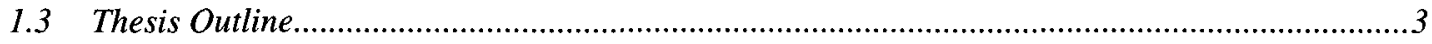

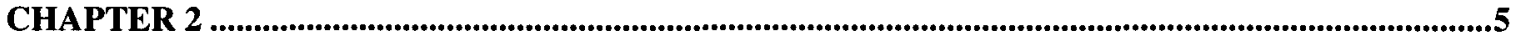

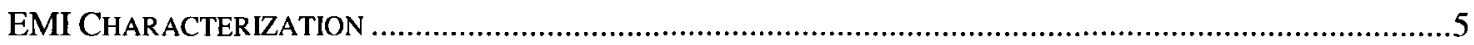

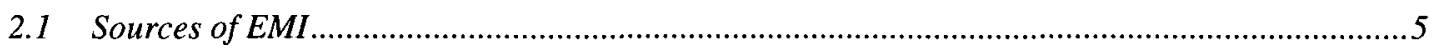

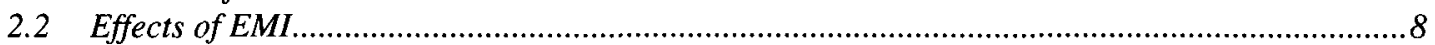

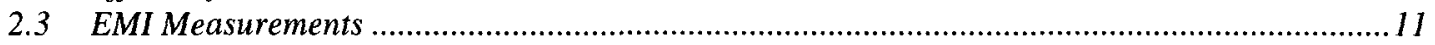

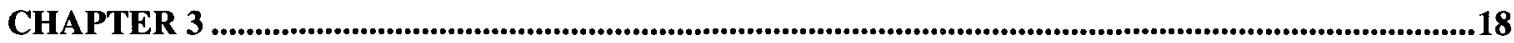

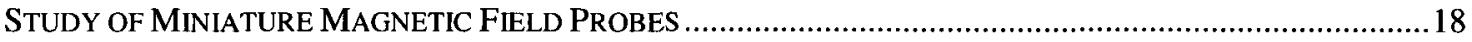

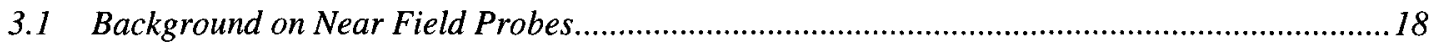

3.2 Novel 3D Probes in High Density Interconnect (HDI) Technology ..........................................22

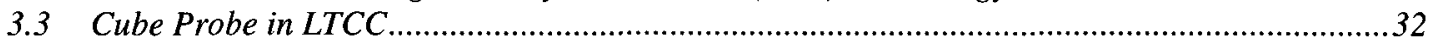

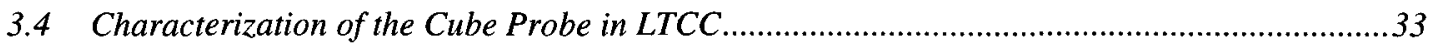

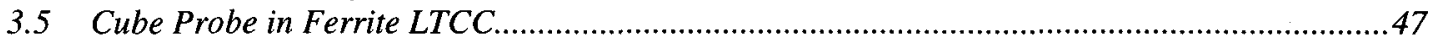

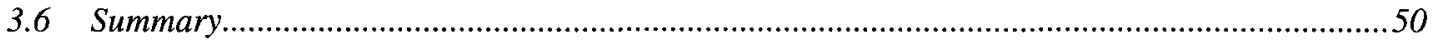

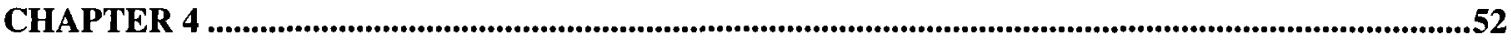

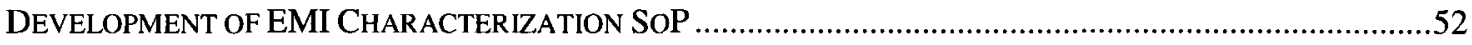

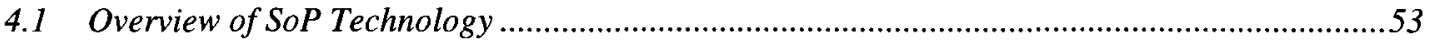

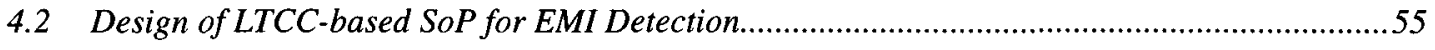

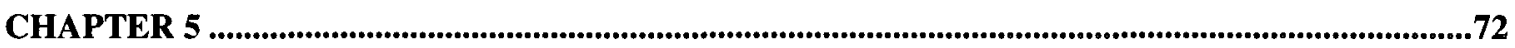

ASSESSMENT/EXPERIMENTAL VALIDATION OF LTCC SOP ...................................................................72

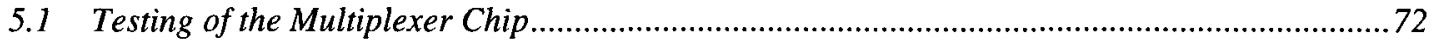

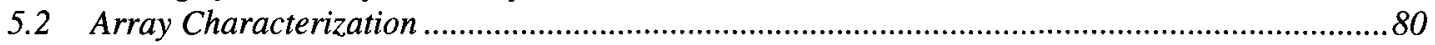

5.3 Package Characterization .................................................................................................. 


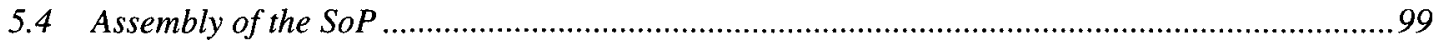

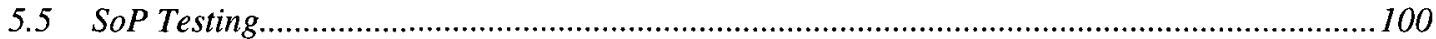

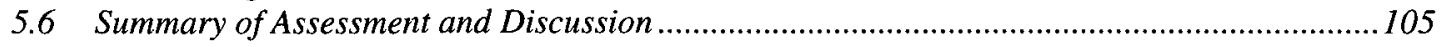

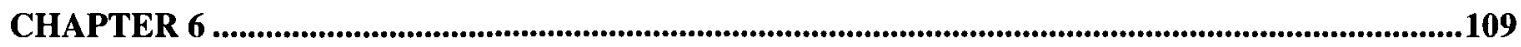

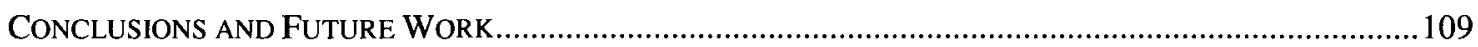

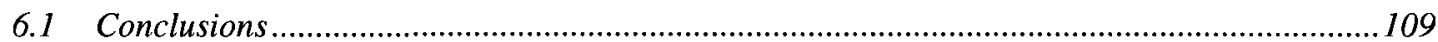

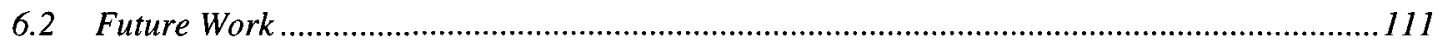

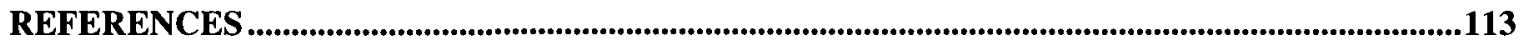




\section{List of Figures}

FIGURE 1-1: EMI SCANNING OF A MICROCONTROLLER CHIP AND ITS EMI RESULTANT MAPPING.....................1 FIGURE 2-1: MOORE'S LAW PROJECTION, AND THE INTEGRATION'S GROWTH OF ELEMENTS IN SYSTEMS [1] ...5 FIGURE 2-2: EXAMPLE OF A HIGH SPEED PULSE TRAIN AND ITS SPECTRUM [3] ..........................................

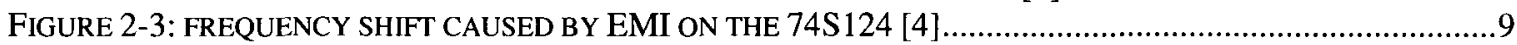

FIGURE 2-4: FREQUENCY SHIFT CAUSED BY EMI ON THE 74LS629 [4] ................................................ 10

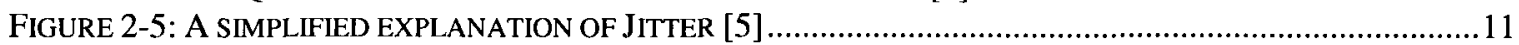

FIGURE 2-6: A) MAGNETIC FIELD PROBE AND B) ELECTRIC FIELD PROBE [7] ...........................................12

FIGURE 2-7: MEASUREMENT AND SIMULATION OF THE TRANSMISSION COEFFICIENT BETWEEN A PROBE AND A

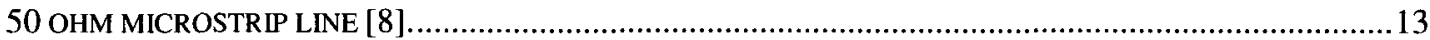

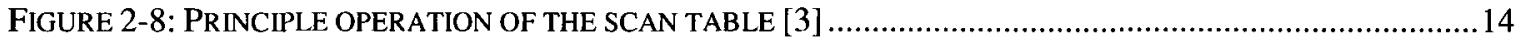

FIGURE 2-9: NEAR FIELD MEASUREMENT FLOW [3] ................................................................................. 16

FiguRE 2-10: PHOTOGRAPH OF A SCAN TABLE LOCATED AT THE TOULOUSE CAMPUS OF FREESCALE

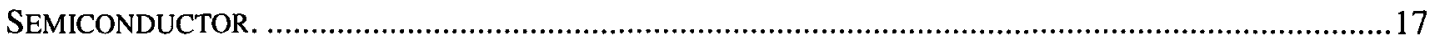

FIGURE 3-1: FUNDAMENTAL LOOP STRUCTURE

FIGURE 3-2: FOUR DIFFERENT INTEGRATED MAGNETIC PROBE STRUCTURES IN HDI: CUBE PROBE STRUCTURE (UPPER left), Tangential Probe Structure (UPPER Right), Normal Probe Structure (LOWer

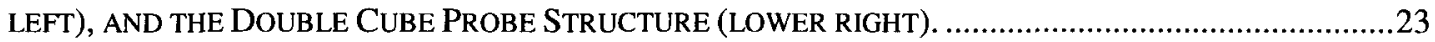

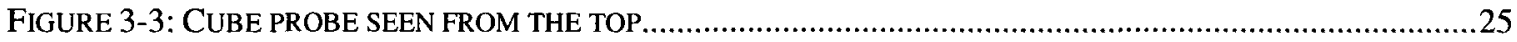

FIGURE 3-4: MAGNITUDE OF THE INPUT IMPEDANCE OF THE FOUR PROBES FABRICATED IN HDI TECHNOLOGY

FIGURE 3-5: EQUIVALENT MODELS FOR 4 NOVEL PROBES: (A) CUBE PROBE, (B) TANGENTIAL PROBE, (C)

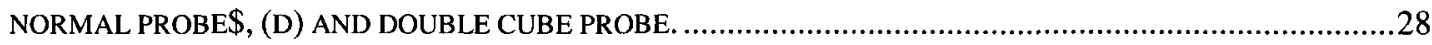

FigURE 3-6: SENSITIVITY TEST OF A CUBE PROBE DONE BY PLACING IT 1 MM ABOVE A MICROSTRIP LINE. ...29

FIGURE 3-7: SENSITIVITY OF PROBES IN TWO ORTHOGONAL ORIENTATIONS: (A) CUBE PROBE, (B) TANGENTIAL

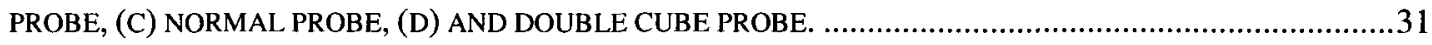

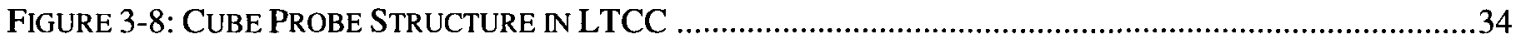

FIGURE 3-9: MAGNITUDE AND PHASE OF THE INPUT IMPEDANCE OF THE CUBE PROBE IN LTCC ....................35

FIGURE 3-10: ELECTRICAL EQUIVALENT MODEL OF A CUBE PROBE SIMULATED IN ANSOFT HFSS .................36

FIGURE 3-11: INPUT IMPEDANCE OF THE CUBE PROBE MEASURED "ON-WAFER" ..........................................38

FIGURE 3-12: EQUIVALENT MODEL OF THE PROBED CUBE PROBE..........................................................38

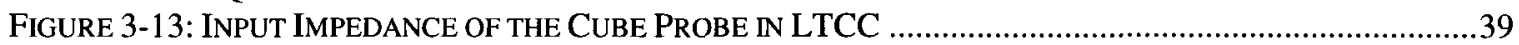

FIGURE 3-14: EQUIVALENT MODEL OF THE CUBE PROBE IN LTCC ......................................................

FIGURE 3-15: CUBE PROBE IN LTCC MAGNETIC FIELD PICK UP WITH RESPECT TO FREQUENCY ….................45

FIGURE 3-16: INPUT IMPEDANCE OF THE DOUBLE CUBE PROBE MEASURED “ON-WAFER”. .............................46

FiguRE 3-17: EQuIVALENT ELECTRICAL MODEL FOR A DOUBLE CUBE PROBE IN LTCC ….........................47

FIGURE 3-18: EQUIVALENT MODEL OF THE FERRITE LTCC CUBE PROBE ….............................................49

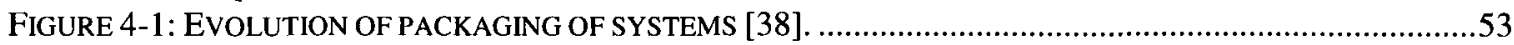

FIGURE 4-2: GRAPHICAL REPRESENTATION OF THE SYSTEM IN THE SOP. A) EACH 8 CUBE PROBES ARE CONNECTED TO ONE MULTIPLEXER. B) ALL THE OUTPUTS OF THE MULTIPLEXERS ARE CONNECTED TO A

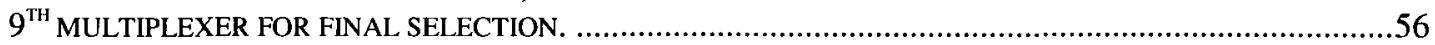

FIGURE 4-3: A) TRANSMISSION GATE, B) EQUIVALENT MODEL OF A TRANSMISSION GATE ...........................59

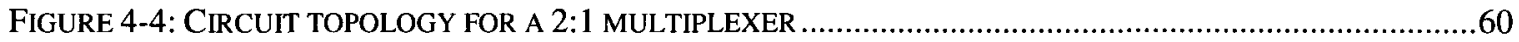

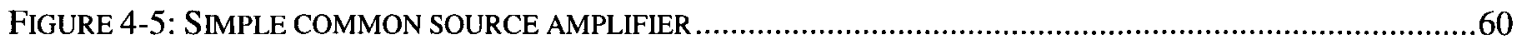

FIGURE 4-6: VARIOUS STAGES THAT FORM THE MULTIPLEXER CHIP.......................................................61

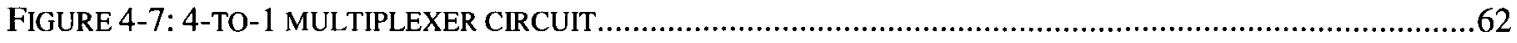

FIGURE 4-8: SCHEMATIC OF THE OUTPUT BUFFER OF THE MULTIPLEXER …….........................................63

FIGURE 4-9: COMPLETE LAYOUT OF THE MULTIPLEXER CHIP SHOWING THE INPUTS AND THE OUTPUT TO THE CHIP.

FIGURE 4-10: S21 PLOT OF THE SIMULATED MULTIPLEXER SCHEMATIC AND EXTRACTED LAYOUT.................65

Figure 4-11: A) CUBE PROBES WITH AN ORIENTATION DIFFERENCE OF 0 , B) CUBE PROBES WITH AN ORIENTATION DIFFERENCE OF 180 
FIGURE 4-12: MUTUAL COUPLING SIMULATION RESULTS SHOWING THE MUTUAL COUPLING IN DB FOR FREQUENCIES BETWEEN 0.1 AND 6 GHZ FOR PROBES IN OPPOSITE ORIENTATION (BLUE) AND SAME ORIENTATION (RED).

FIGURE 4-13: CUBE PROBES PLACED IN THE LTCC LAYOUT. .........................................................69

FIGURE 4-14: COMPLETE SOP LAYOUT SHOWING THE PADS FOR THE VARIOUS EXTERNAL CONNECTIONS .....70

FIGURE 4-15: FABRICATED LTCC MODULE POPULATED WITH MULTIPLEXER CHIPS .................................71

FIGURE 5-1: PACKAGED CHIP CLAMPED TO A CFP80TF TEST FIXTURE .................................................73

FIGURE 5-2: WIRE BONDING PLAN SUBMITTED TO CMC IDENTIFYING THE WIRE-BONDED PINS...................74

FIGURE 5-3: MEASURED $S_{11}$ OF THE MULTIPLEXER INPUT PORT THROUGH THE TEST FIXTURE. .....................75

FIGURE 5-4: MEASURED $S_{21}$ OF THE MULTIPLEXER CHIP THROUGH THE TEST FIXTURE ..............................76

FIGURE 5-5: COMPARISON OF THE INSERTION GAIN OF THE MULTIPLEXER CHIP'S BUFFER WITH AND WITHOUT

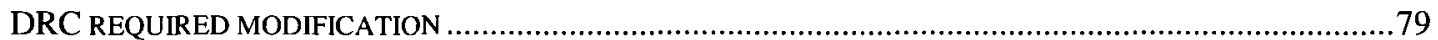

FIGURE 5-6: PICK UP OF AN ISOLATED CUBE PROBE (PROBE PLACED AT Z=1 MM, $X=1 \mathrm{MM}$ ) ........................82

FIGURE 5-7: CUBE PROBE PICKUP IN A 9 ELEMENT ARRAY FOR PROBE PLACED AT $Z=1 \mathrm{MM}, \mathrm{X}=1 \mathrm{MM}$ (RED) AND

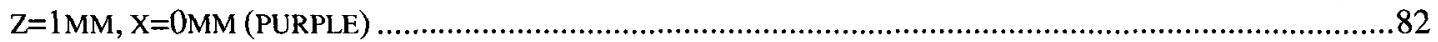

FIGURE 5-8: TWO ARRAY TEST STRUCTURES BUILT IN LTCC WITH SHORT CIRCUITED CUBE PROBES (UPPER

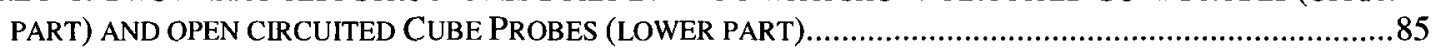

FIGURE 5-9: EQUIVALENT MODEL OF A CUBE PROBE IN THE MIDDLE OF AN ARRAY OF SHORTED SIMILAR PROBES.

FIGURE 5-10: MEASUREMENT DERIVED EQUIVALENT MODEL OF THE CUBE PROBE IN THE MIDDLE OF AN ARRAY OF SIMLAR SHORTED PROBES

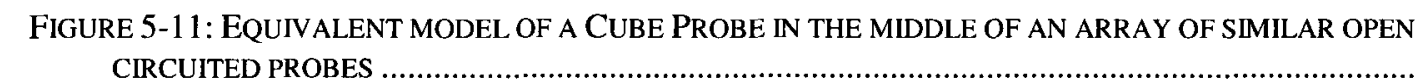

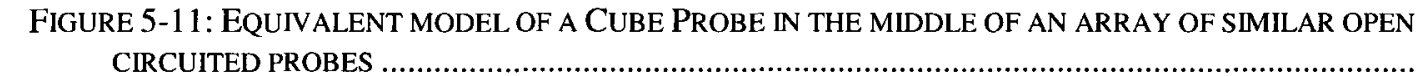

FIGURE 5-12: EQUIVALENT MODEL OF THE CUBE PROBE PLACED IN THE MIDDLE OF AN ARRAY OF SIMILAR

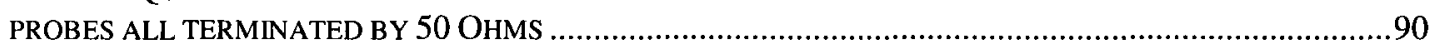

FIGURE 5-13: STRUCTURE SIMULATED FOR MUTUAL COUPLING …................................................92

FIGURE 5-14: MUTUAL COUPLING BETWEEN TWO NEIGHBOURING CUBE PROBES SEPARATED BY 1 MM......93

FIGURE 5-15: MUTUAL COUPLING AT THE INPUT PORTS OF A MULTIPLEXER CHIP...................................94

FIGURE 5-16: THE SIMULATED STRUCTURE FOR MUTUAL COUPLING WITH THE INSERTED GROUND PLANE IN

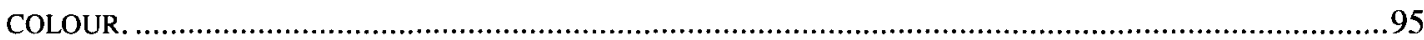

FIGURE 5-17: MUTUAL COUPLING AT THE MULTIPLEXER CHIP LEVEL AFTER THE INTRODUCTION OF THE

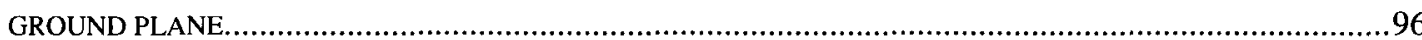

FIGURE 5-18: MUTUAL COUPLING AT THE MULTIPLEXER CHIP LEVEL AFTER THE INTRODUCTION OF GROUND

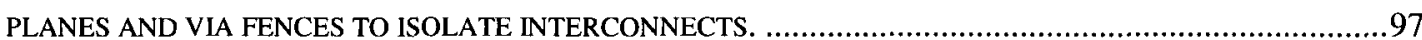

FIGURE 5-19: INSERTION LOSS OF THE TRANSMISSION LINE FROM THE CUBE PROBE TO THE MULTIPLEXER

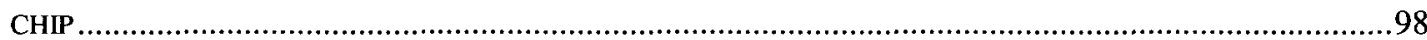

FIGURE 5-20: INSERTION LOSS OF THE TRANSMISSION LINE FROM A PERIPHERAL CHIP TO THE NINTH

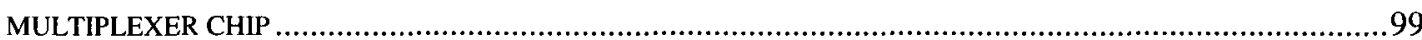

FIGURE 5-21: SPECTRUM ANALYZER OUTPUT MARKING THE SIGNAL LEVEL AT $850 \mathrm{MHZ}$ FOR THE FIRST TEST

FIGURE 5-22: SPECTRUM ANALYZER OUTPUT MARKING THE SIGNAL LEVEL AT 880 MHZ FOR THE FIRST TEST

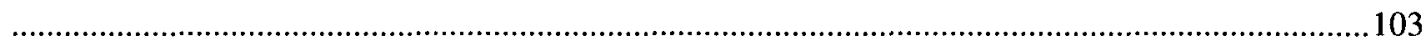

FIGURE 5-23: SPECTRUM ANALYZER OUTPUT MARKING THE SIGNAL LEVEL AT 850 MHZ FOR THE SECOND TEST.

FIGURE 5-24: SPECTRUM ANALYZER OUTPUT MARKING THE SIGNAL LEVEL AT 880 MHZ FOR THE SECOND TEST.

FIGURE 5-25: INSERTION LOSS OF THE SYSTEM MEASURING EMI AT 1 MM OVER A MICROSTRIP LINE WHEN THE OUTPUT BUFFER OF THE CHIP IS NOT WORKING

FIGURE 5-26: INSERTION LOSS OF THE SYSTEM EMI AT 1 MM OVER A MICROSTRIP LINE WHEN THE OUTPUT BUFFER IS WORKING 


\section{List of Abbreviations and Symbols}

$\begin{array}{ll}\text { EMC } & \text { Electromagnetic Compatibility } \\ \text { EMI } & \text { Electromagnetic Interference } \\ \text { LTCC } & \text { Low Temperature Co-fired Ceramic } \\ \text { HDI } & \text { High Density Interconnect } \\ \text { FR4 } & \text { Flammable Retardant 4 } \\ \text { PCB } & \text { Printed Circuit Board } \\ \text { SoP } & \text { System on Package } \\ \text { IOO } & \text { Input / Output } \\ \text { CMC } & \text { Canadian Microelectronics Corporation } \\ \text { Q } & \text { Quality Factor } \\ V_{d d} & \text { Supply Voltage } \\ S_{11} & \text { Input return loss } \\ S_{21} & \text { Transmission gain } \\ \text { CMOS } & \text { Complementary Metal Oxide Semiconductor } \\ \text { NMOS } & \text { N-channel Metal Oxide Semiconductor } \\ \text { PMOS } & \text { P-channel Metal Oxide Semiconductor } \\ C_{o x} & \text { Oxide Capacitance } \\ R_{d s} & \text { Channel Resistance } \\ \text { L } & \text { Gate Length or Inductance } \\ \mathrm{W} & \text { Gate Width } \\ \mathrm{G}_{\mathrm{m}} & \text { Transconductance } \\ \mathrm{R}_{\mathrm{out}} & \text { Output Resistance }\end{array}$




\section{Chapter 1}

\section{Introduction}

\subsection{Motivation}

As electronic integrated circuits become smaller and operate at higher frequencies, the effect of electromagnetic interference (EMI) on their performance and meeting electromagnetic compatibility (EMC) specifications and regulations is increasingly more important. The testing of circuit level EMI often requires the use of near field magnetic field loops placed over the device under test (DUT). The loop is displaced over the DUT by means of a scan table which can accurately move the loop in all $3 D$ directions. The magnetic field strength measured by the magnetic loops is then received by either a spectrum analyzer or an EMC analyzer, or treated in the time domain which increases the measurement's speed.

An example of an EMI measurement system and the EMI mapping result (for a 2 $\mathrm{cm} \times 2 \mathrm{~cm}$ packaged microcontroller chip) is given in Figure 1-1. The photos in Figure 1-1 were taken at the Freescale campus in Toulouse, France
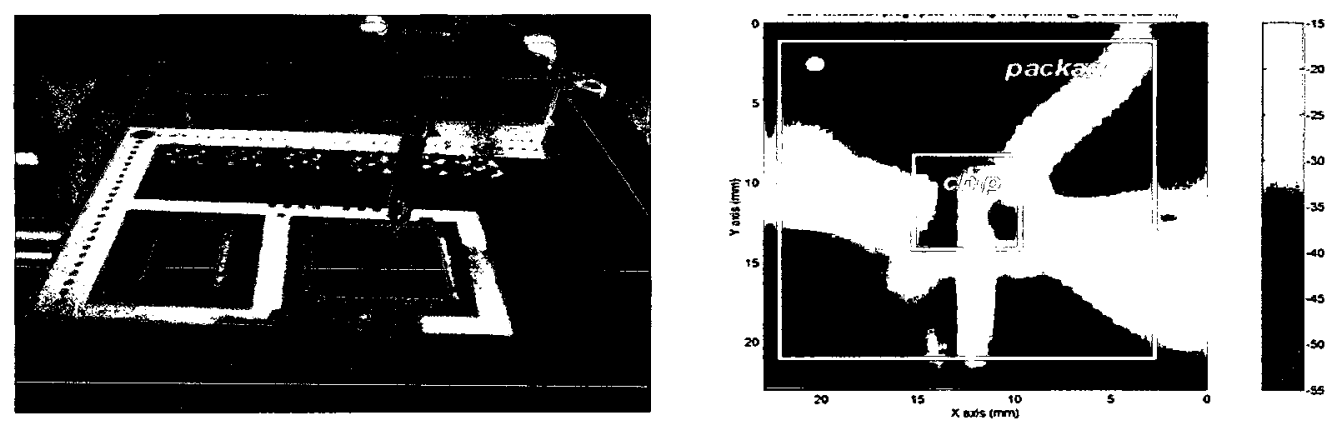

Figure 1-1: EMI scanning of a microcontroller chip and its EMI resultant mapping 
The shrinkage of the gate length of transistors, and hence of integrated circuits, is coupled with the need for smaller magnetic loops to increase the sensitivity of EMI measurements and to accurately locate emission zones. One way to accomplish this is through the design of miniature probes integrated within a PCB. Such probes, if placed in an array and connected to active circuitry, allowing their individual interrogation, could offer a revolutionary tool for the measurement of EMI. The resulting system would remove the need for a scan table. In relation to this concept, low temperature co-fired ceramic (LTCC) have recently emerged as an elegant packaging solution for more robust and condensed designs. . When a package is used to have not only active chips and their interconnects, but also embedded passives such as antennas, the complete module is called System on Package (SoP).

\subsection{Thesis Objective}

The overall goal of this research is to explore the design and implementation of a novel LTCC based SoP capable of performing EMI measurements over the 0.1 $\mathrm{GHz}$ to $3 \mathrm{GHz}$ band while eliminating the need for a scan table. In particular, the system should provide adequate spatial resolution and transfer the detected magnetic field captured by the cube probes to a spectrum analyzer. Specifically, the goals of this thesis are as follows:

- Characterize novel PCB and LTCC based miniature magnetic field probes and determine their equivalent models and their sensitivity. 
- Carry out a custom multiplexer chip design for use in the EMI diagnostics tool.

- Develop a custom LTCC SoP containing and array of probes and the multiplexer chips.

- Perform experimental validation of the complete EMI characterization SoP.

\subsection{Thesis Outline}

The work in this thesis revolves around the characterization of new EMI measuring probes and using them in an array to replace the operation of scanning with scan tables.

In chapter 2, the problem of EMI is presented from a background perspective. Chapter 2 looks at the future trends in technology and specifically in miniaturization and in the reduction of gate lengths. The chapter presents the problem of EMC/EMI as becoming more important, and how engineers are in need of novel EMI characterization tools.

Chapter 3 is dedicated to the characterization of novel EMI probes that measure emitted magnetic fields. Four different probes are investigated, of which the Cube Probe and the Double Cube Probe emerge as superior candidates for EMI measurements. The chapter then focuses on the characterization of the cube probes in LTCC and ferrite LTCC for increased integration and enhanced sensitivity. 
Introduction

Chapter 4 introduces the system on package SoP designed for the integration of an array of cube probes with active circuitry. A design of a multiplexer chip, and of a package in LTCC that holds 64 cube probes are discussed. The chapter discusses the implementation of the SoP and how it can be a good replacement of the scan table to create a handy EMI characterization tool for engineers.

In Chapter 5, an assessment is presented of all the elements that form the SoP. The chapter initially speaks about the performance of the multiplexer chip. Then, the chapter focuses on the behaviour of the cube probe when surrounded by other cube probes. The characterization of the interconnects and the transmission lines in the SoP is also presented in chapter 5 . Finally, the SoP test results are presented.

The last chapter, chapter 6 , presents the conclusions observed from the work in the thesis and recommends future work that needs to be implemented to enhance the performance of the SoP and to present a working product for EMC/EMI engineers. 


\section{Chapter 2}

\section{EMI Characterization}

This chapter provides a brief overview of the problem of electromagnetic interference EMI, its roots, and its effect. This chapter also discusses EMI measurements and the advancement in near-field scanning.

\subsection{Sources of EMI}

Every modern integrated circuit IC is composed of multiple transistors switching on and off at various times. In the last 40 years, the complexity of ICs has increased significantly with the reduction in transistor sizes. In accordance with Moore's law, where the number of transistors per chip has increased exponentially, the number of components in a system has increased significantly per $\mathrm{cm}^{2}$ as shown in Figure 2-1 [1].

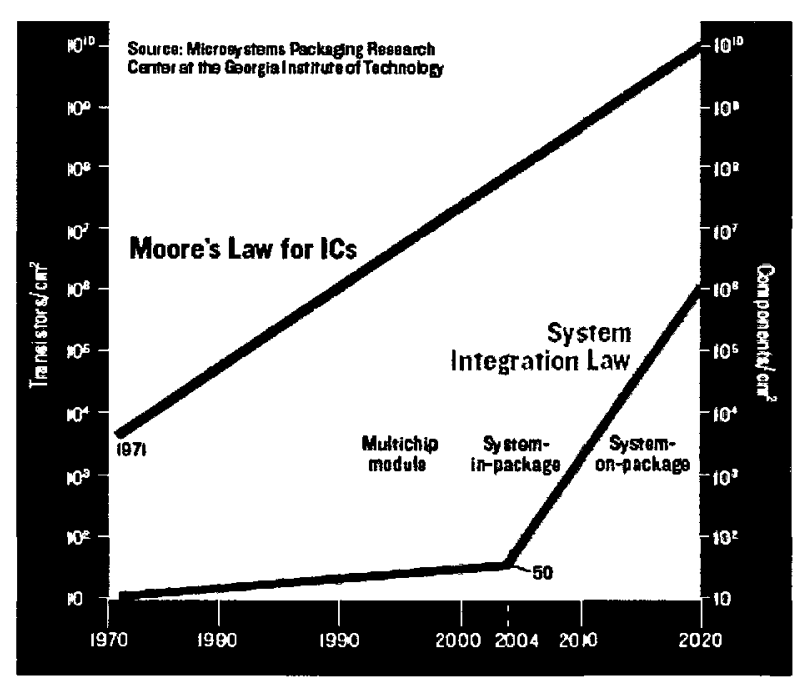

Figure 2-1: Moore's law projection, and the Integration's growth of elements in systems [1]. 
EMI Characterization

6

The increase in the number of elements in an IC, has forced the reduction of supply voltage to minimize power dissipation. However, due to the high integration, the peak currents have increased considerably per layout area as shown in Table 2-1 [2].

\begin{tabular}{|l|l|l|l|l|l|}
\hline Technology & $\begin{array}{l}\text { Voltage } \\
\text { Rail }(\mathrm{V})\end{array}$ & $\begin{array}{l}\text { Density of } \\
\text { cells } / \mathbf{m m}^{2}\end{array}$ & $\begin{array}{l}\text { Current per } \\
\text { gate (mA) }\end{array}$ & $\begin{array}{l}\text { Capacitance } \\
\text { per gate (fF) }\end{array}$ & $\begin{array}{l}\text { Current per } \\
\text { area }\left(\mathbf{A} / \mathbf{m m}^{2}\right)\end{array}$ \\
\hline $1.2 \mu \mathrm{m}$ & 5 & $8 \mathrm{~K}$ & 1.1 & 60 & 8.8 \\
\hline $0.8 \mu \mathrm{m}$ & 5 & $15 \mathrm{~K}$ & 0.9 & 40 & 13.5 \\
\hline $0.5 \mu \mathrm{m}$ & 5 & $28 \mathrm{~K}$ & 0.75 & 30 & 21 \\
\hline $0.35 \mu \mathrm{m}$ & $5-3.3$ & $50 \mathrm{~K}$ & 0.6 & 25 & 30 \\
\hline $0.25 \mu \mathrm{m}$ & $5-2.5$ & $90 \mathrm{~K}$ & 0.4 & 20 & 36 \\
\hline $0.18 \mu \mathrm{m}$ & $3.3-2$ & $160 \mathrm{~K}$ & 0.3 & 15 & 48 \\
\hline $0.12 \mu \mathrm{m}$ & $2.5-1.2$ & $240 \mathrm{~K}$ & 0.2 & 10 & 48 \\
\hline $90 \mathrm{~nm}$ & $2.5-1$ & $480 \mathrm{~K}$ & 0.1 & 7 & 48 \\
\hline $65 \mathrm{~nm}$ & $2.5-0.8$ & $1000 \mathrm{~K}$ & 0.07 & 5 & 50 \\
\hline $45 \mathrm{~nm}$ & $1.8-0.8$ & $2000 \mathrm{~K}$ & 0.05 & 3 & 55 \\
\hline
\end{tabular}

Table 2-1: Evolution of current amplitudes in CMOS technologies [2]

While the current peaks have increased per layout area, the major noise contributors are typically the same in every system:

- The digital blocks require a sharp transition of current at the clock edge. As the minimum size gate length decreases and the microprocessors hold more transistors, the noise generated by the switching of logic gates in digital cores is increasing. 
- As the number of $\mathrm{I} / \mathrm{O}$ port increases, the generated noise increases since it requires a significant amount of current for CMOS transistors acting as passing gates to be turned on or off. As the number of $\mathrm{I} / \mathrm{O}$ ports that are turned off and on at the same time is higher, the generated noise will be higher.

- The voltage regulators, of various types that are transferring charges with every clock cycle.

- The noise on the clock signal is distributed to the whole digital core, which propagates the noise in the whole system.

- Some analog circuits like PLLs or power amplifiers that can produce parasitic signals in determined frequency bands. [3]

The generation of noise at specific time intervals is responsible for the creation of noise across a large frequency spectrum. Figure 2-2 shows the Fast Fourrier Transform (FFT) of a high speed pulse train with a frequency of $10 \mathrm{MHz}$ and a 2 ns rise and fall times. 
Fouriertransform - Example

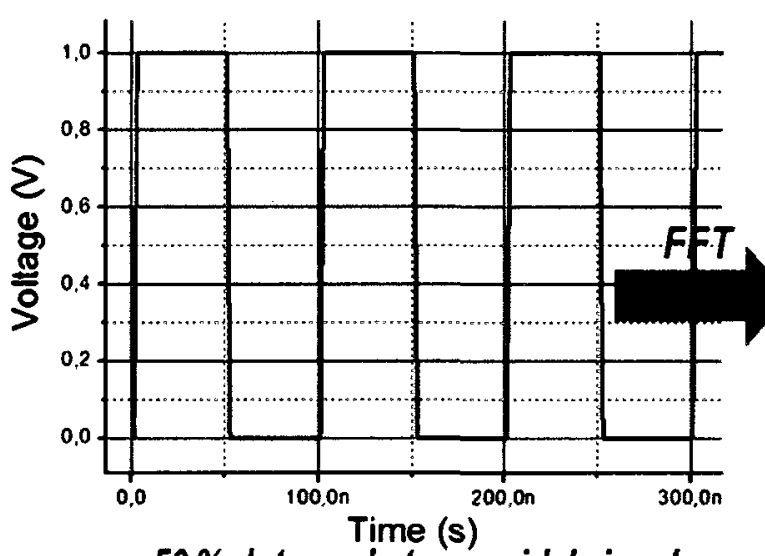

$50 \%$ duty cycle trapezoidal signal

Period $T=100 \mathrm{~ns}, T_{r}=T_{f}=2 \mathrm{~ns}$

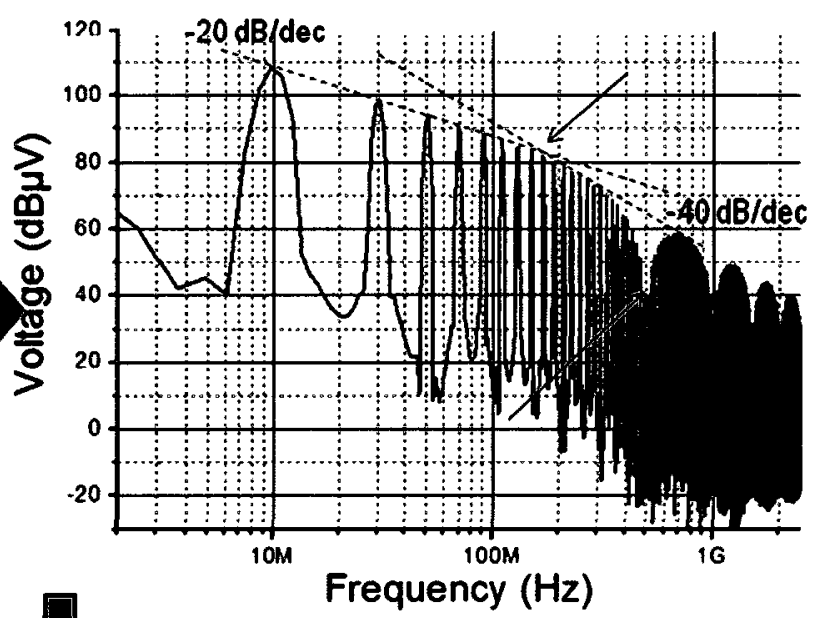

Evaluation of signal bandwidth

Figure 2-2: Example of a high speed pulse train and its spectrum [3]

\subsection{Effects of EMI}

While this thesis is primarily concerned with circuit-level EMI emissions, this section will highlight some of the issues in EMI susceptibility of circuits. Various standards for EMC have been put in place, to minimize the effect of electromagnetic interference EMI. This is a clear cut evidence of the damaging effect that EMC and EMI can share in the failure of systems. These standards have been established for all the electronics industries; from the automotive industry to home appliances, to handheld devices and telecommunications...etc. This section looks into some particular effects that EMI has on digital and analog circuits. 


\subsubsection{EMI Effect on Oscillators}

A study has shown that EMI has a significant effect on the crystal oscillators responsible for setting reference frequencies in VCOs for digital and analog circuits. The oscillators were exposed to an applied near field signal by a loop antenna. The deviation in frequency was observed at the crystal oscillator level causing malfunctioning in the digital system relying on the VCO output frequency. The study was performed on the 74LS629 and 74S124 VCO chips, made by Texas Instruments. The frequency spectrum outputs of both chips are shown in Figures 2-3 and 2-4 where the deviations in frequency are significant. It should be noted that the voltage measured at the disturbed oscillators of $91.2 \mathrm{mV}$ rms was responsible for the shift in frequency. [4]

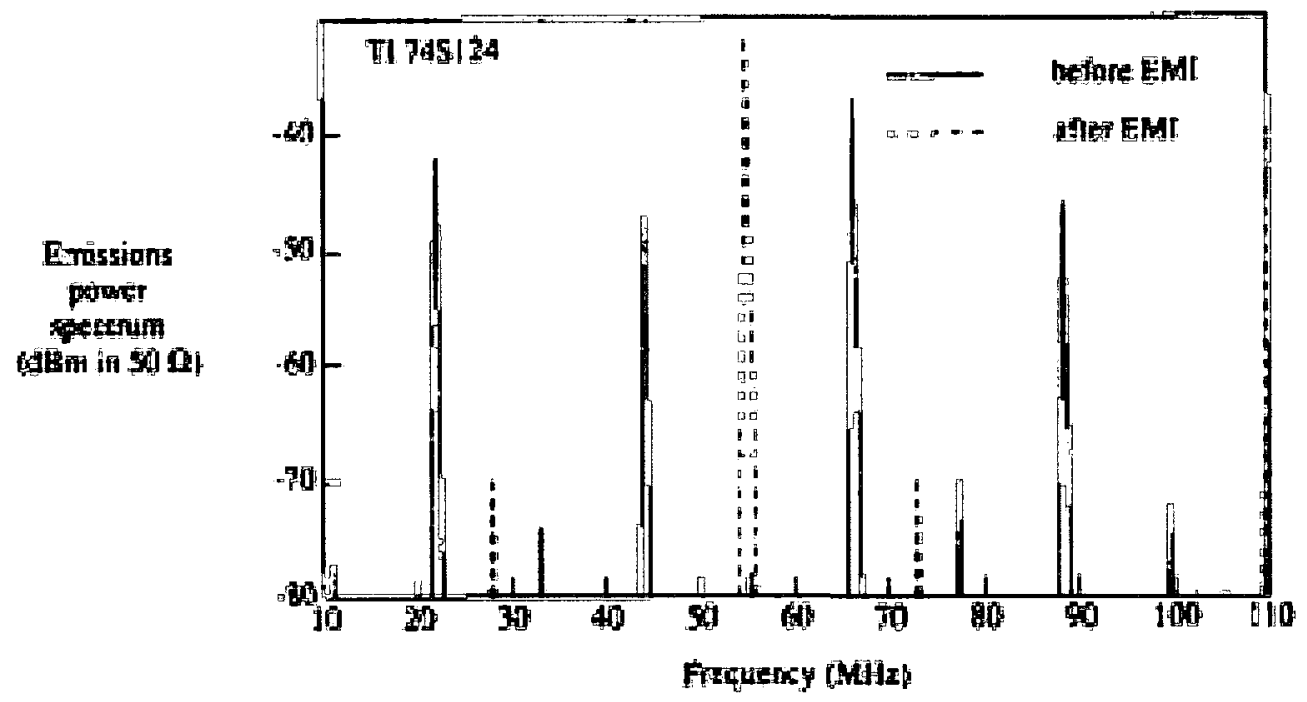

Figure 2-3: frequency shift caused by EMI on the 74S124 [4] 


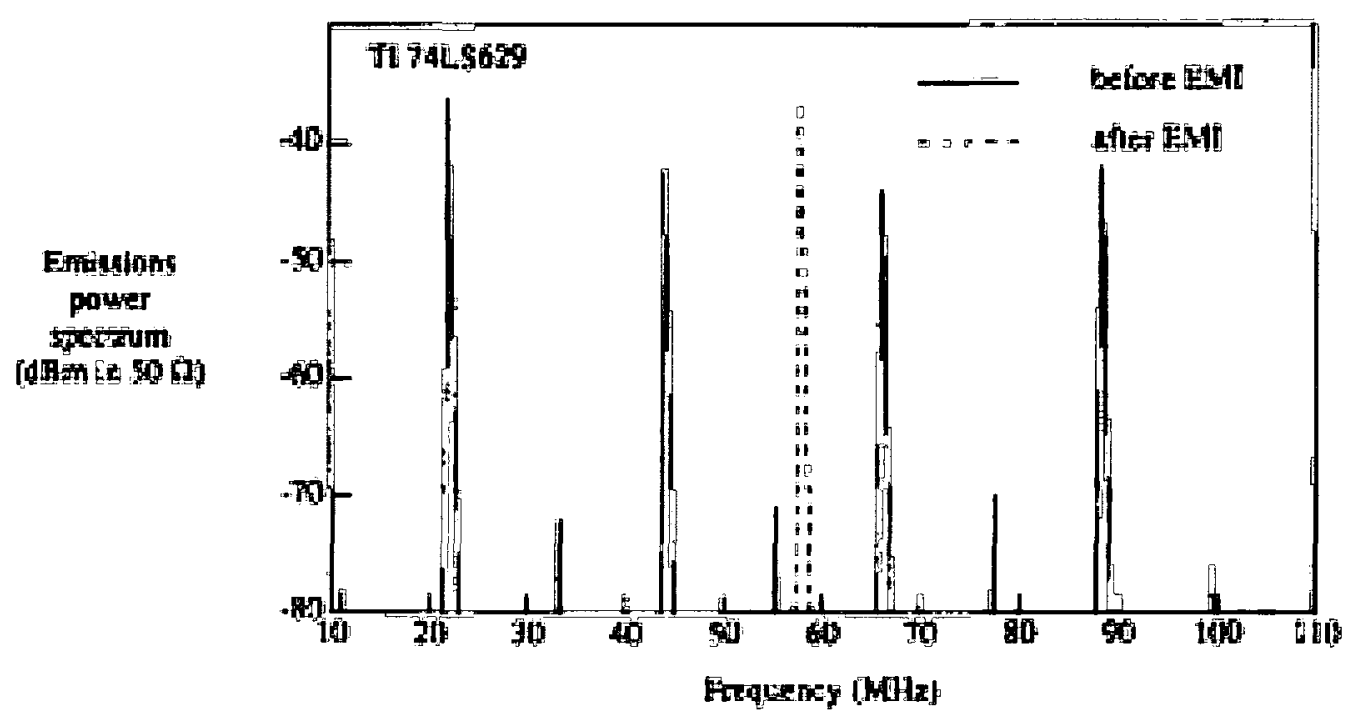

Figure 2-4: frequency shift caused by EMI on the 74LS629 [4]

\subsubsection{EMI-induced Jitter}

EMI is also responsible for inducing jitter in digital circuits. This phenomenon known as EMI-induced jitter can occur with minimal EMI levels, leading to irregularities in the operation of digital circuits and false switching. While the effect of jitter is dependent on the timing constraints in a given digital circuit, it can lead to the failure of certain circuits. The amount of jitter induced in a logic device can be quantified based on the known value of the EMI-induced RMS voltage. Figure 2-5 shows the effect of jitter on a given logic signal with both time $\left(\mathrm{T}_{\mathrm{j}}\right)$ and amplitude $\left(\mathrm{V}_{\mathrm{pp}}\right)$ noise effects present [5].

$V_{p p}$ is the peak to peak induced threat voltage, $T_{r}$ is the rise time for a given digital pulse, and $A$ is the pulse amplitude. 


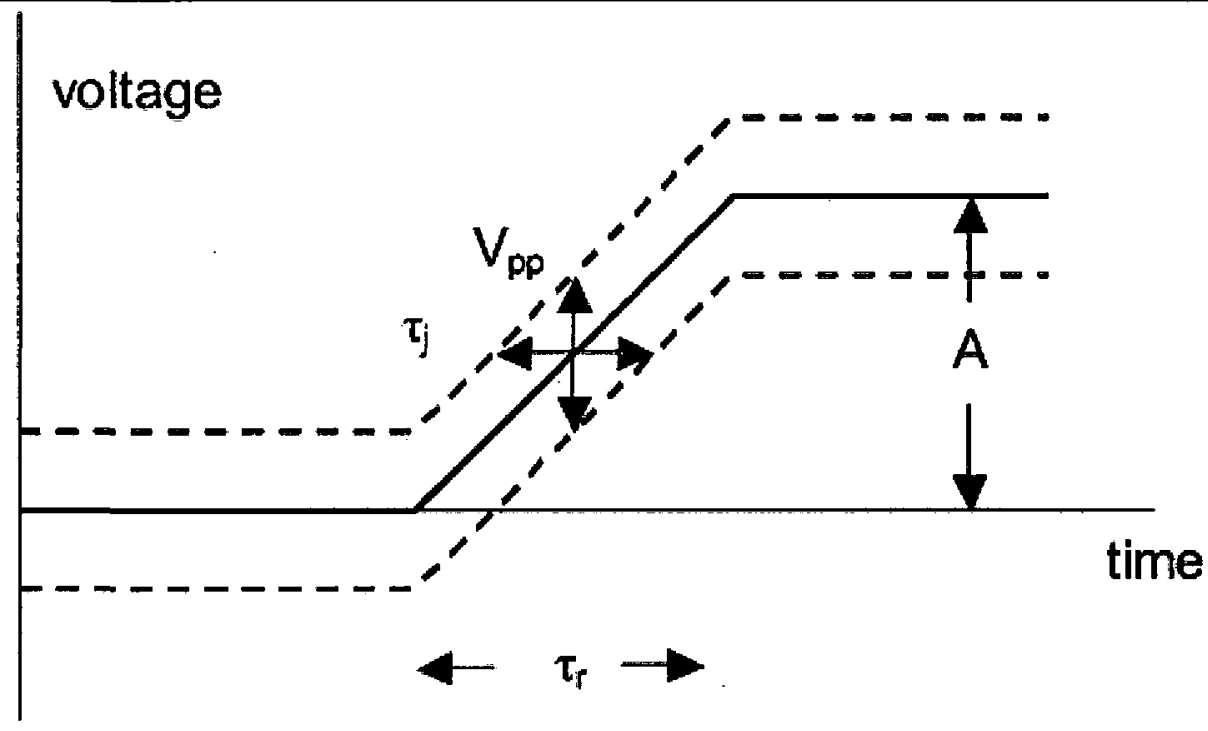

Figure 2-5: A simplified explanation of Jitter [5]

\subsection{EMI Measurements}

While it is known that EMI is responsible for causing circuit failures, knowing the sources that generate EMI on a given circuit is even more crucial. Some developed software tools are relying on the formation of simple current dipoles and standard core noise simulation to predict the radiated near field above the surface of an integrated circuit with good accuracy. One of these tools is IC-EMC developed by the LATTIS group at INSA de Toulouse, in Toulouse France. While work is ongoing in the development of simulation tools and circuit models that predict emissions on a given integrated circuit, it is still not complete and complex enough to predict the EMC behaviour of complex system in package circuits. [6] 


\subsubsection{Magnetic Field Probes}

The need arises for physical tools to measure EMI on the surface level of integrated circuits. For this reason, multiple probes were developed for the measurement of near-field magnetic and electric fields. Most of the near-field probes are made of coaxial cables by looping the centre conductor and forming miniature loop antennas. Some other loops are formed by constructing patterns on PCB material. Figures 2-6a and 2-6b show a magnetic near-field probe, and an electric near field probe respectively. [7]

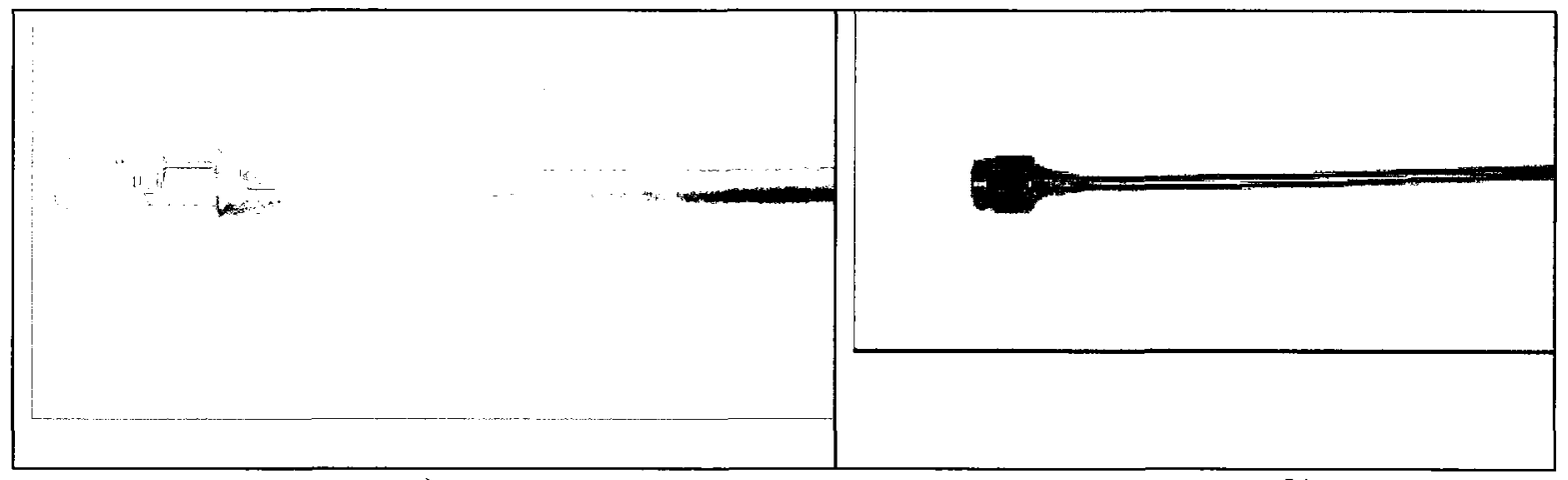

a)

b)

Figure 2-6: a) Magnetic field probe and b) Electric field probe [7]

While the development of near-field probes is necessary for EMI measurements, an important feature is needed to efficiently measure EMI radiated by an integrated circuit. Near field probes have to be miniature enough to be able to measure and determine areas of emissions on VLSI circuits. Therefore the size of the probe should not exceed a few mms in every direction. For VLSI circuits, the loop diameter of a magnetic probe should not exceed $5 \mathrm{~mm}$, since the resonant frequency of the loop should be in the tens of $\mathrm{GHz}$. The coupling between the DUT and the loop has to be inductive, and therefore can be 
EMI Characterization 13 modeled as a mutual inductance between the probe and a package lead or wire bond. To validate the claim of inductive coupling, some simulation was done for a loop placed at $1 \mathrm{~mm}$ above a $50 \mathrm{ohm}$ microstrip line. The same setup was physically constructed and an $S_{21}$ was measured between the loop and the transmission line. The results confirmed that a loop acts like an inductor until about $6 \mathrm{GHz}$, where the coupling mechanism seems to change. Figure 2-7 shows the test setup and the simulation and measurement results. [8]

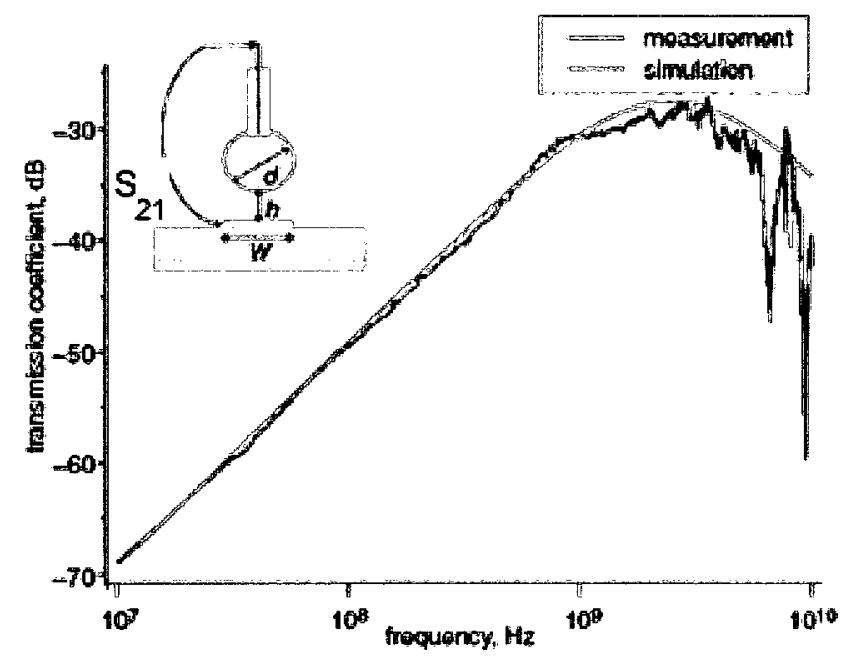

Figure 2-7: Measurement and simulation of the transmission coefficient between a probe and a $50 \mathrm{ohm}$ microstrip line [8].

\subsubsection{Scan Tables}

To form a mapping of EMI radiation from a DUT, a magnetic probe should be displaced over an integrated circuit with fine steps. EMC researchers at Daimler Chrysler developed a scanning mechanism that uses three axis stepper motors to achieve a resolution of $400-600 \mu \mathrm{m}$. This way the probe is displaced above 
EMI Characterization 14 an integrated circuit and the fields are measured for every probe location. $A$ mapping of the results is then possible showing the spots of highest emissions on the device under test DUT. [9]

The principle operation of the scan table and the test setup is shown in Figure 28. The magnetic field probe is suspended on top of the device under test. The probe is mechanically displaced over the DUT, and the measured magnetic field is transmitted via coaxial cables to a spectrum analyzer. A computer is connected to the spectrum analyzer to save the data for each probe displacement.

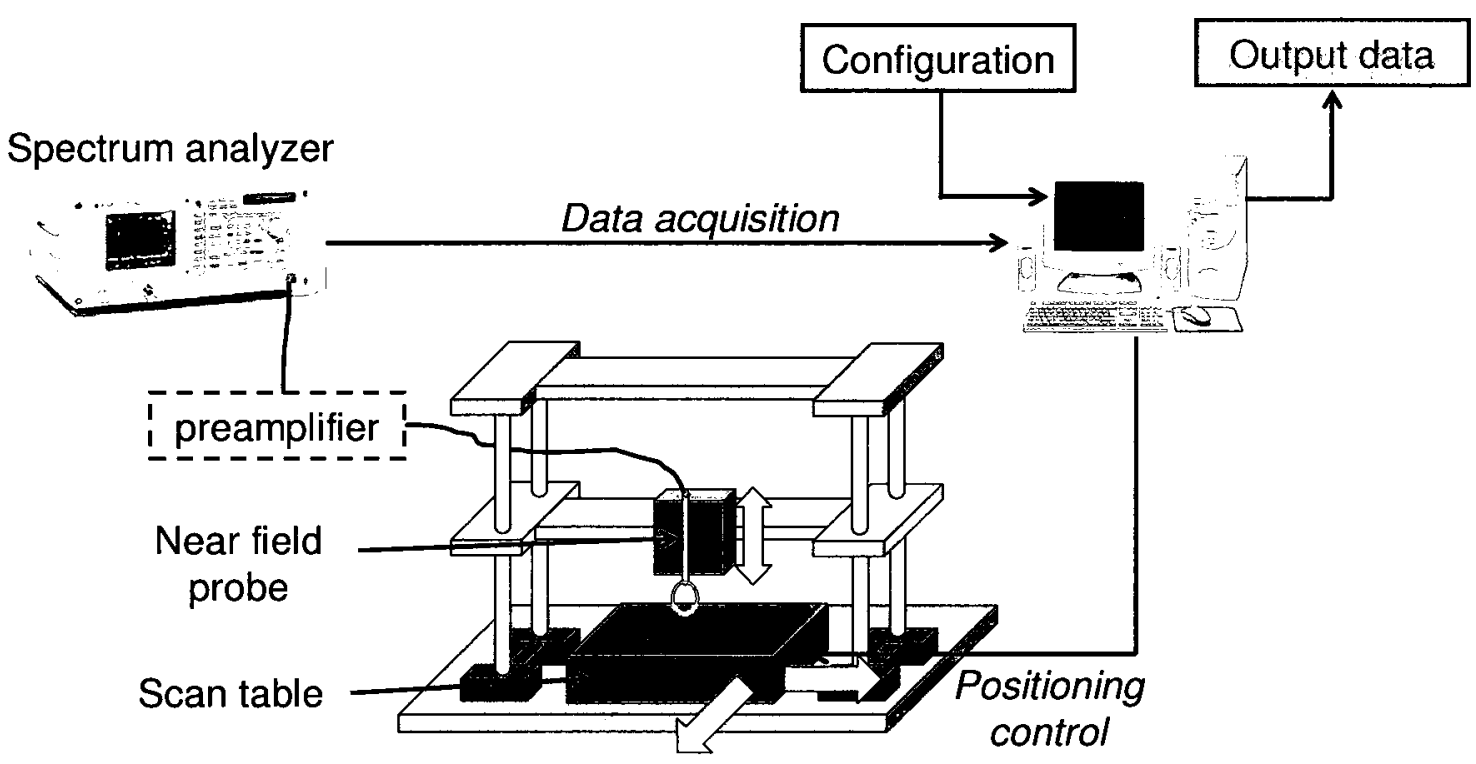

Figure 2-8: Principle operation of the scan table [3]

For the scan table to execute properly, an algorithm was developed to control the motion of the magnetic field probe. The algorithm starts by having the start and stop positions picked for the probe. All the frequency bands necessary for the measurements are also picked. This way a spectrum analyzer can be controlled 
EMI Characterization 15 to have the right center frequency, span and video and resolution bandwidths set to ensure a sound measurement, with low noise. When the EMI measurements start, the probe is placed in the start position, and the algorithm ensures that the magnetic field is measured at all required frequency components. Then, the position of the probe is shifted to the next position, and the measurements are repeated for every displacement. The algorithm checks at every position if it is the stop position in order to finish the test. When the stop position is reached, all the data is available for all the points for the various frequencies. Thus, EMI emission maps can be produced. The algorithm and a sample EMI emissions map are shown in Figure 2-9. [3]

Figure $2-10$ is of an actual photograph taken of a scan table located at the Toulouse campus of Freescale Semiconductor in France. 


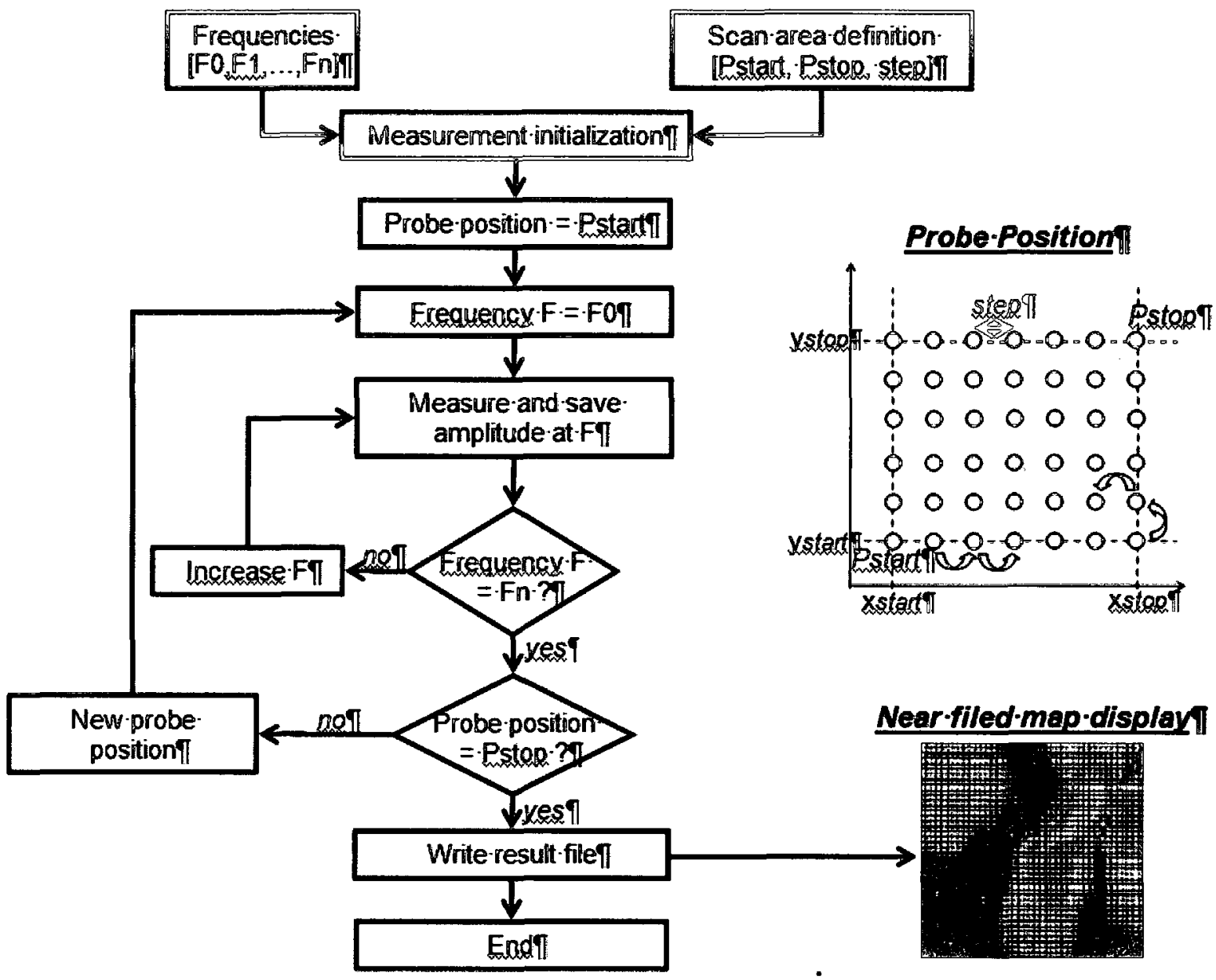

Figure 2-9: Near field measurement flow [3] 


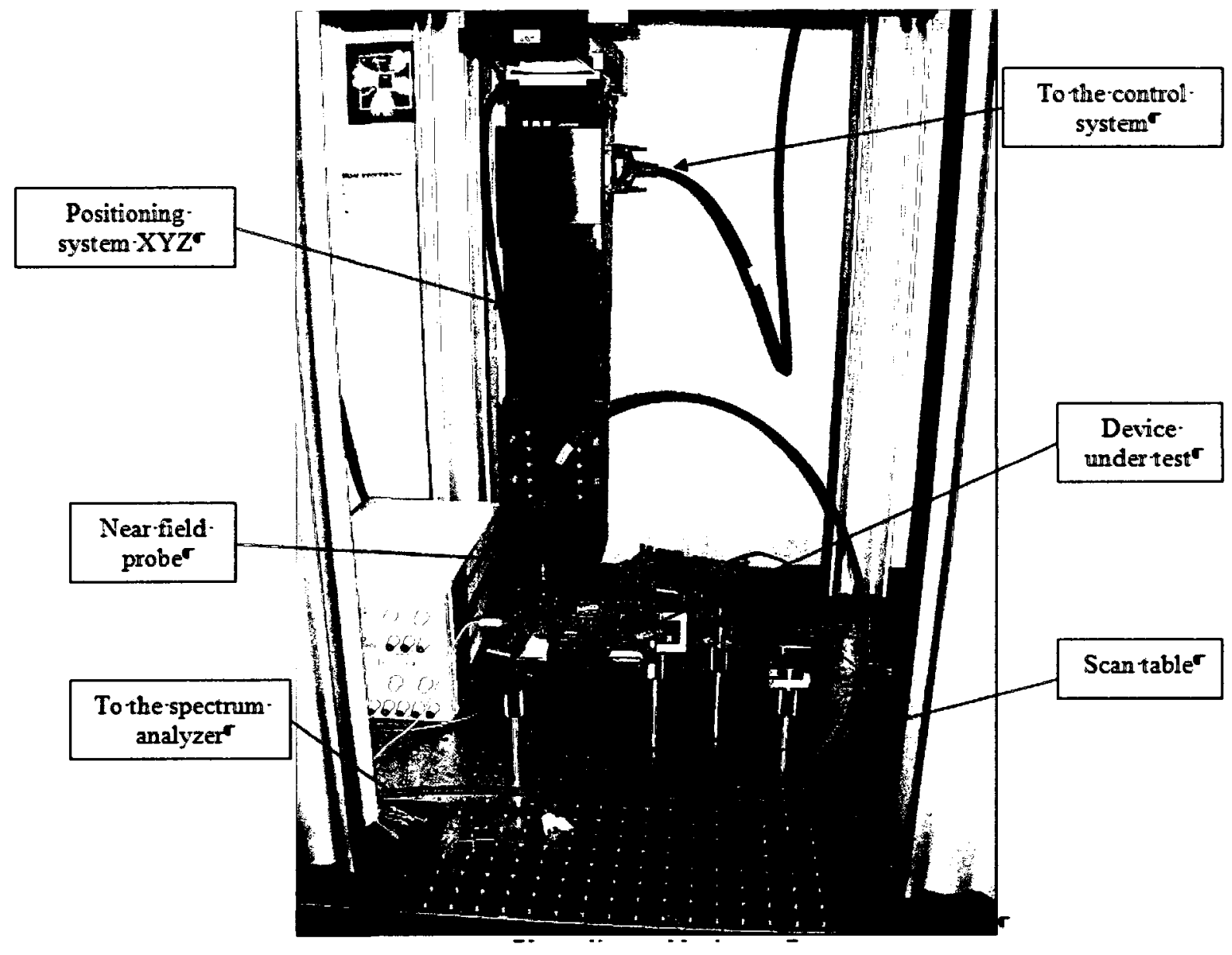

Figure 2-10: Photograph of a scan table located at the Toulouse campus of Freescale Semiconductor. 


\section{Chapter 3}

\section{Study of Miniature Magnetic Field Probes}

As mentioned in Chapter 2 of this thesis, the source of EMI emissions is mostly I/O ports. The currents of switched circuits cause magnetic fields to become significant calling for EMI measurements. Therefore, magnetic field probes are deemed more important than electric field probes in measuring EMI. Since the coupling of magnetic field is done inductively, a large mutual inductance between a probe and a device under test is a good indication of magnetic field detection. Therefore, the main design criterion for the development of magnetic field probes is to increase the inductance. However, when combined with other parasitic elements in the magnetic field probe structure, a higher inductance or a higher parasitic capacitance will lead to a lower resonant frequency; limiting the operation frequency of the probe frequency wise. Based on the above, when examining magnetic field probe structures, their equivalent model can predict their effectiveness when it comes to energy pickup and the high frequency operation.

In this chapter, we will characterize novel magnetic field probes and determine their equivalent models and their sensitivity with respect to frequency.

\subsection{Background on Near Field Probes}

The design of near field scanning probes is important in terms of the probe's frequency response and adapting it to measure radiation or emission in a 
particular frequency band. Generally, such probes are desired to have a wide frequency response especially for EMI measurements. The most basic type of magnetic field probe is a loop formed by the inner conductor of a coax cable as shown in Figure 2-6.

Its fundamental operation can be understood by considering the simple loop geometry shown in Figure 3-1.

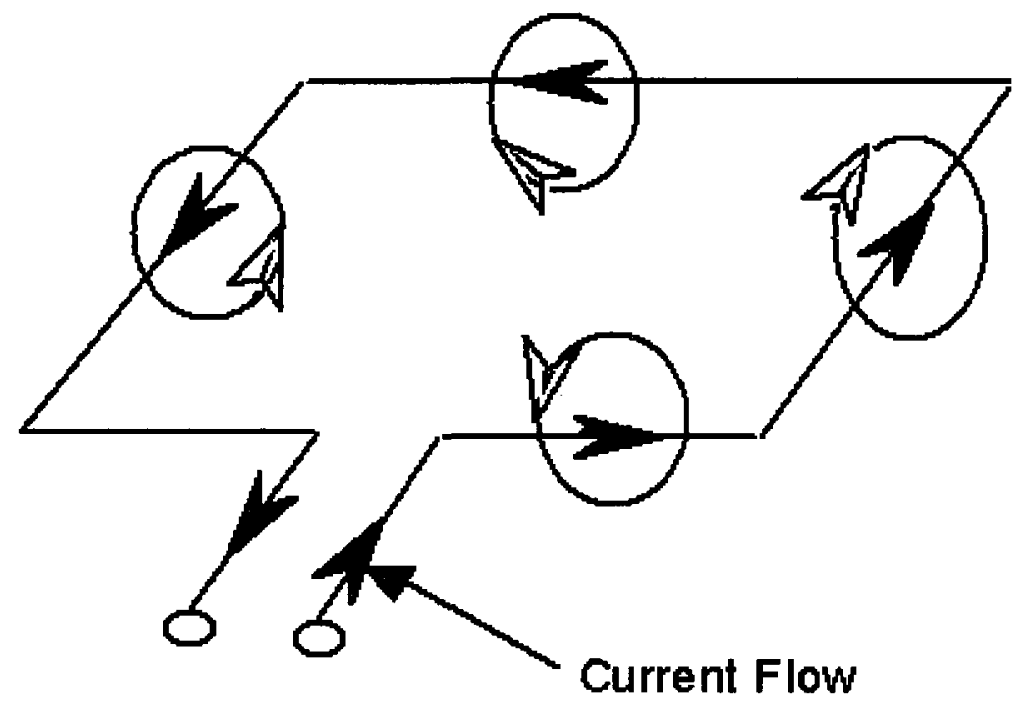

Figure 3-1: Fundamental loop structure.

The relationship between an induced flux in the presence of an incident magnetic field, normal to the plane of the loop, is derived by Equation (3-1). $S$ is the surface area of the loop in question. It should be noted that the loop in question should have dimensions significantly less than the wavelength of the frequency of the applied magnetic field.

$$
\psi=\int_{s} B \cdot d s
$$

The inductance is defined as the ratio of the induced flux to the generated current in a given structure. With $\mathrm{N}$ loops (as the one shown in Figure 3-1) connected, 
Study of Miniature Magnetic Field Probes 20 the linkage flux between the loops and the self induced flux in each loop contribute to an inductance given by Equation 3-2, as a function of the square of the number of loops $\mathrm{N}$.

$$
L=N^{2} \frac{\psi}{I}
$$

Therefore, based on the findings of Equations (3-1) and (3-2), we can deduce that the geometry of the loop controls the amount of flux induced in the loop, and that the inductance of the loop itself will control the current flow in the loop. Furthermore, the magnetic energy stored in the loop is given by Equation (3-3). Therefore, a loop with a higher inductance will lead to the storage of more magnetic energy and therefore allow for better detection of magnetic fields. [10]

$$
W_{m}=\frac{1}{2} L I^{2}
$$

Although a loop in itself is a very simple structure to measure magnetic fields, probes that measure electric field have also been developed. In 1980, Dahele and Cullen similarly constructed a semirigid coax cable to create a field probe to measure the electric field on a microstrip line. The use of the inner conductor of the coax cable as the probe, with the outer conductor providing shielding, was found to be the best geometry for near-field probing. [11]

Subsequent papers have used coax probes to determine microwave properties of structures [12], and specifically at high microwave frequencies [13]. The use of open-ended coaxial probes has been extended to determine the dielectric constant of tissues for use in the bio-medical field [14], and the permittivity of lossy materials [15]. 
Study of Miniature Magnetic Field Probes

In [16], various techniques were established for the design of both electric field and magnetic field probes. In some cases, dipoles used to sense electric fields were capacitively loaded, or resistively loaded, or even loaded with diodes to achieve a stable frequency response over very large bands. The same technique was applied to loops sensing magnetic fields. In [17], a probe was developed for measuring near-field electric and magnetic fields simultaneously, which enables the calculation of instantaneous Poynting vectors describing the energy flow. In [18] improvements were made to the probe developed in [17] to take into account the effect of unmatched loads making it suitable for frequency swept measurements. Other H-field loops, developed in [19], have been designed to measure magnetic fields in the head (when talking on a cellphone). However, none of the probes has the spatial resolution required to measure EMI from small VLSI circuits. Furthermore, to achieve sensing in all 3 dimensions, the miniature antennas must be used in threes and their outputs added using combiners.

Miniature probes have also been developed for the measurement of emissions of integrated circuits, such as in [20], where a miniature magnetic loop, built on a dielectric substrate, is utilized to measure magnetic fields in one plane at a time although not providing a high spatial resolution. In [21] a thin film miniaturized magnetic field probe is developed to measure EMI on integrated circuits. The spatial resolution is very high (in the order of $40 \mu \mathrm{m}$ ), but the complexity of the probes is significant. Each probe is sandwiched between a photoresist insulator layer and a conductor layer on both sides and the observed resonance is at 2 
Study of Miniature Magnetic Field Probes 22 $\mathrm{GHz}$. In addition the probe can only measure emitted magnetic field in one plane at a time only.

A higher spatial resolution can be obtained when fiber optical probes and systems are utilized such as in [22],[23], [24], and [25].However the complexity of the measuring systems is quite substantial, employing both fiber optics and RF technologies.

\subsection{Novel 3D Probes in High Density Interconnect}

\section{(HDI) Technology}

The motivation behind the newly developed magnetic probe structures in this thesis is the need for integration of miniature magnetic probes with other electronic circuitry. The concept of the new magnetic probes is based on completely embedding the probes within a printed circuit board (PCB) or other packaging and simultaneously sensing all three directional components of the magnetic field. The aim is to create simple structures that are miniature enough so as not to perturb the circuits under test and provide adequate spatial resolution. The probes should work in the 0.1 to $4 \mathrm{GHz}$ range, which corresponds to the frequencies of emissions from digital circuits, and microprocessors specifically. The construction of the probes requires the creation of loops with conductive lines in 2 different layers, joined by vias from one layer to the other. Figure 3-2 shows various probe structures developed with INSA de Toulouse using a PCB (HDI) technology. [26] [7] 


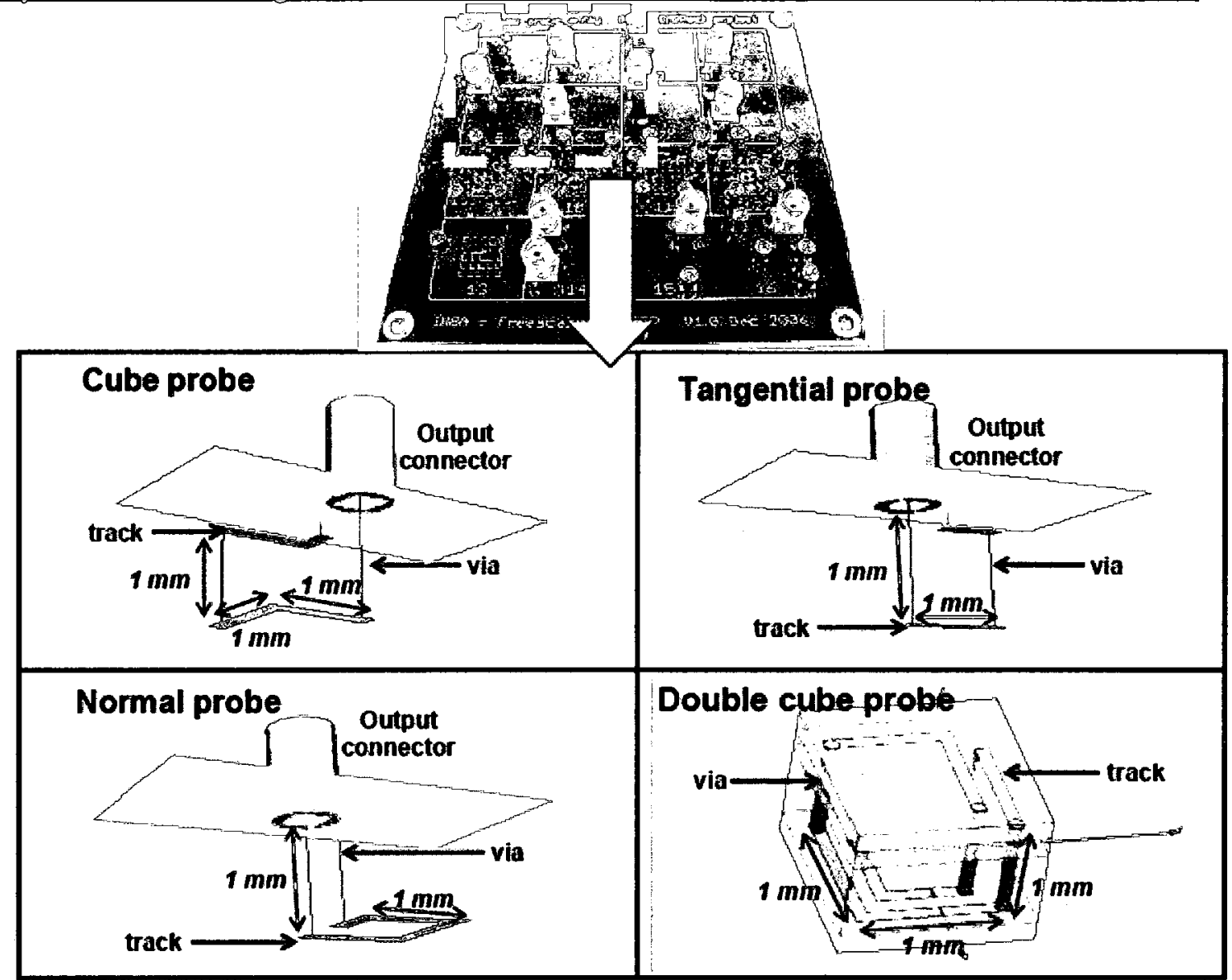

Figure 3-2: four different integrated magnetic probe structures in HDI: Cube Probe

Structure (upper left), Tangential Probe Structure (upper right), Normal Probe Structure (lower left), and the Double Cube Probe Structure (lower right).

The four designs built and characterized are the following:

- Cube Probe: a novel design able to pick up magnetic field in all 3 dimensions. The probe is a 3D loop formed by following 6 connected edges of a cube as shown in Figure 3-2. The structure collapses to a loop in each of the $X Y, X Z$, and $Y Z$ planes. Thus according to Equation 3, this probe will respond to $\mathrm{Hx}, \mathrm{Hy}$ and $\mathrm{Hz}$ field components. 
Study of Miniature Magnetic Field Probes

- Tangential Probe: a loop that is able to pick up the fields tangential to the plane of the device under test DUT (used as a first reference design). Assuming the loop to be in the ZY plane, the output connector to be along the axis $Z$, and the DUT to be in the XY plane, then this loop will respond to Hy.

- Normal Probe: a loop that is able to pick up the fields normal to the plane of the device under test DUT (used as a second reference design). Assuming the loop to be in the $X Y$ plane, the output connector to be along the axis $Z$, and the DUT to be in the XY plane, then this loop will respond to $\mathrm{Hz}$.

- Double Cube Probe: An enhancement of the cube probe in that it is a regular cube probe connected to an internal smaller cube probe. The inductance of the double cube probe is roughly twice of the cube probe, which doubles the flux coupling, and therefore enhances the sensitivity of the probe.

In order to achieve adequate spatial resolution, all the probes are defined to have a length of conductor of $1 \mathrm{~mm}$ in all the $\mathrm{X}, \mathrm{Y}$, and $\mathrm{Z}$ directions; having an area of $1 \mathrm{~mm}^{2}$ in each plane. The metal tracks are $100 \mu \mathrm{m}$ wide and the vias have a diameter of $150 \mu \mathrm{m}$. Figure 3-3 shows the cube probe as seen from the top. 


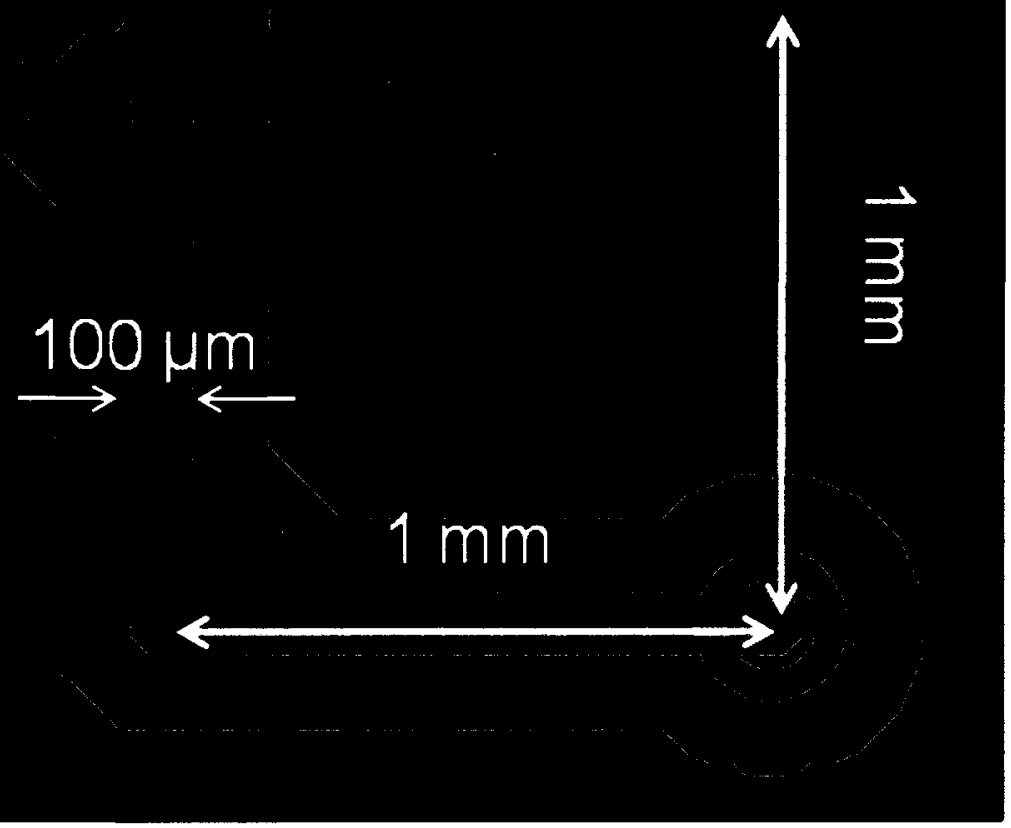

Figure 3-3: Cube probe seen from the top.

The probes are fabricated in a 2-layer printed circuit board technology called $\mathrm{HDI}$, in Toulouse France. The material has identical properties to Flame Retardant 4 (FR4) technology. The fabrication guideline in HDI is given in [26]. Figure 3-2 shows the PCB in which the 4 probes are realized. The probes and their SMB type connectors are surrounded by the red traces in Figure 3-2.

\subsubsection{Characterization of the Probes}

The measured input impedance magnitudes of the 4 probes are displayed on Figure 3-4 as a function of frequency. According to Equation (3), the probes have an inductance $L$, giving rise to an impedance $Z=j \omega L$, whose magnitude increases 
Study of Miniature Magnetic Field Probes

with frequency. This is clearly shown in Figure 3-4 although up to a certain frequency. Resonance effects are seen to occur around $2 \mathrm{GHz}$ for the double cube probe and close to $4 \mathrm{GHz}$ for the tangential probe with the other two probes resonating in between those frequencies.

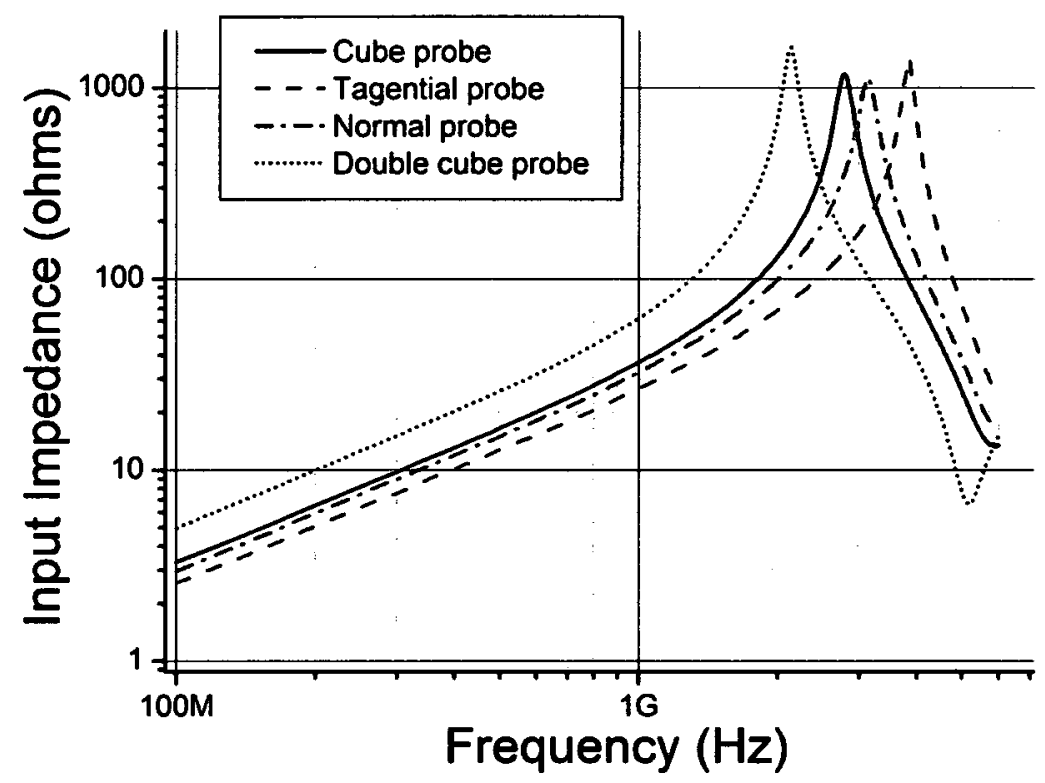

Figure 3-4: Magnitude of the input impedance of the four probes fabricated in HDI technology

These effects can be explained by using an equivalent model derived from input impedance measurements for each of the four probes. In addition to an inductance $L$, the probe structures will have:

- Shunt capacitance associated with the metal lines and their spacing.

- Series and shunt resistors associated with the conductivities of the metal and dielectric, respectively. 
Study of Miniature Magnetic Field Probes 27 Figure 3-5 shows the equivalent models that resulted from the measurements. The models clearly show highest inductance $(10 \mathrm{nH})$ for the double cube probe, which is the desired result. The second highest inductance $(5 \mathrm{nH})$ is associated with the cube probe, which is also expected given its longer conductor length than the remaining two structures. The tangential loop, having the shortest conductor length, has the lowest inductance $(4 \mathrm{nH})$. The models also show the parasitic capacitances to vary as expected, with the structures having the longest closely spaced conductors exhibiting the highest capacitance. The series resistances are found to be negligible and the shunt resistors provide a good match with measurements as shown in Figure 3-11 later in the chapter. 
Study of Miniature Magnetic Field Probes 28

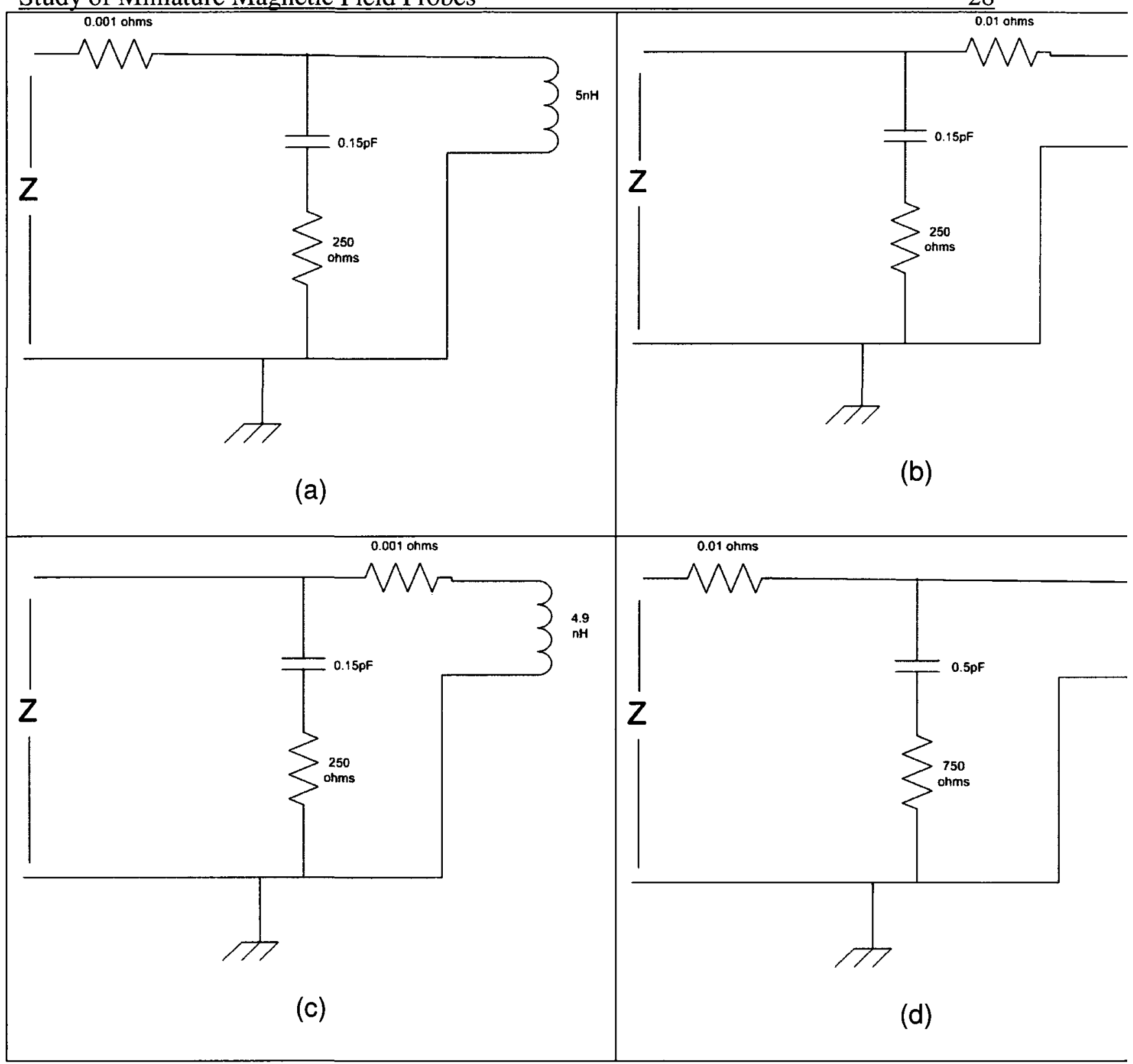

Figure 3-5: Equivalent models for 4 novel probes: (a) Cube probe, (b) tangential probe, (c) normal probe $\$$, (d) and double cube probe.

While the models are a good indication of the performance of the probes (in terms of inductance and parasitic), they do not take into account the sensitivity associated with the geometry of a probe. For example, the cube probe is sensitive to magnetic field in all three planes, while the tangential and normal 
Study of Miniature Magnetic Field Probes

probes are not. Therefore, a test of sensitivity is required by detecting the fields emitted from a simple EMI source. In this case, a microstrip transmission line is chosen (width $=5 \mathrm{~mm}$, on a Rogers Duroid 5880 board). All the probes are placed $1 \mathrm{~mm}$ above the $50 \mathrm{ohm}$ strip. The microstrip is terminated by a load of 50 ohms to minimize the standing waves, thereby eliminating any errors pertaining to positioning. On the other end of the microstrip line, port 1 of a network analyzer is connected, while port 2 is connected to the output of the probes as shown in Figure 3-6 for the case of a Cube Probe.

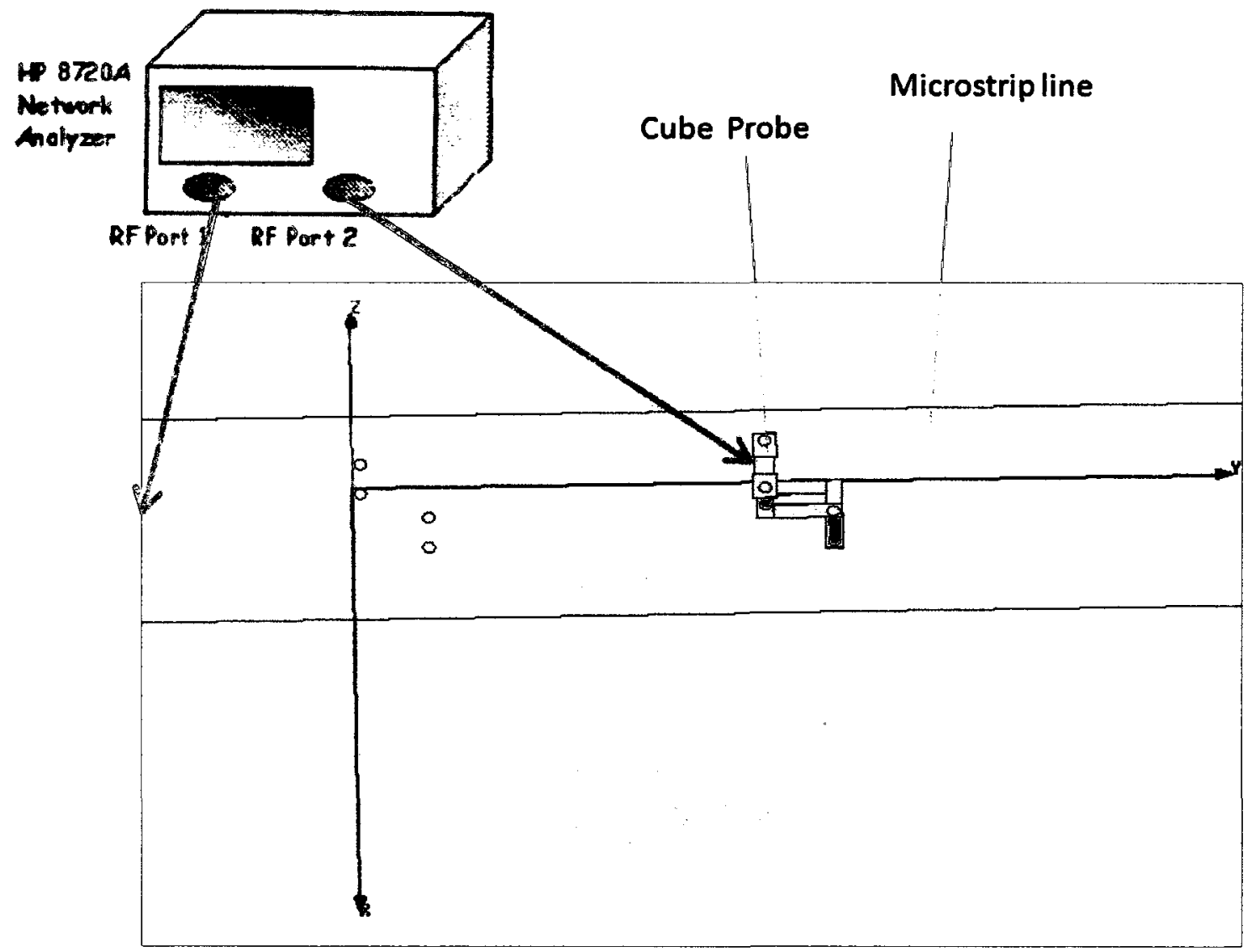

Figure 3-6: Sensitivity test of a Cube Probe done by placing it $1 \mathrm{~mm}$ above a microstrip line. 
Study of Miniature Magnetic Field Probes

30

An $S_{21}$ transmission measurement allows us to characterize the relative sensitivity (coupling) of each probe. The measurements are conducted between $100 \mathrm{MHz}$ and $6 \mathrm{GHz}$ and for two orthogonal orientations of the probes with respect to microstrip EMI source. Figure 3-7 shows the measured sensitivity responses. 
Study of Miniature Magnetic Field Probes

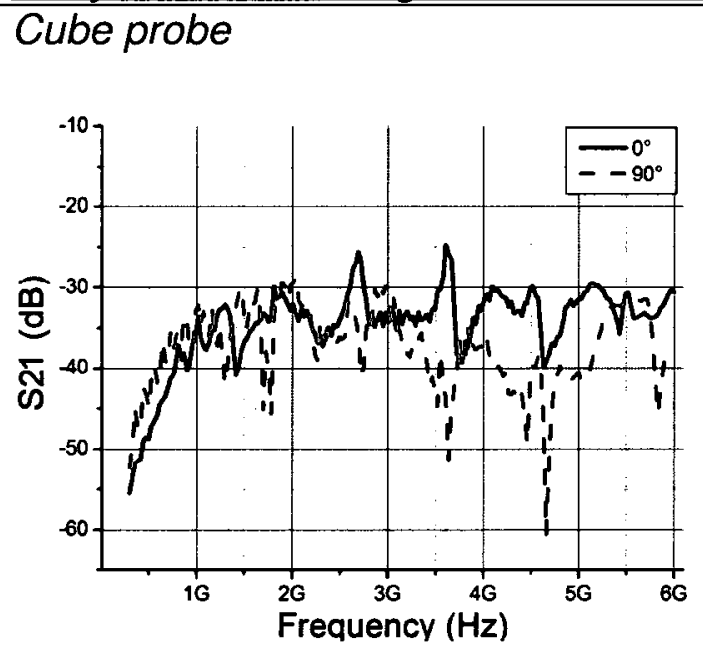

(a)

\section{Normal probe}

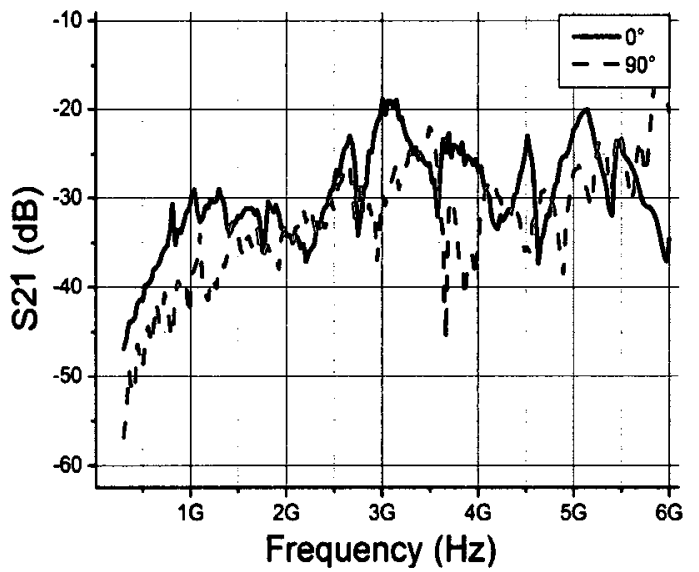

(c)

\section{Tangential probe}

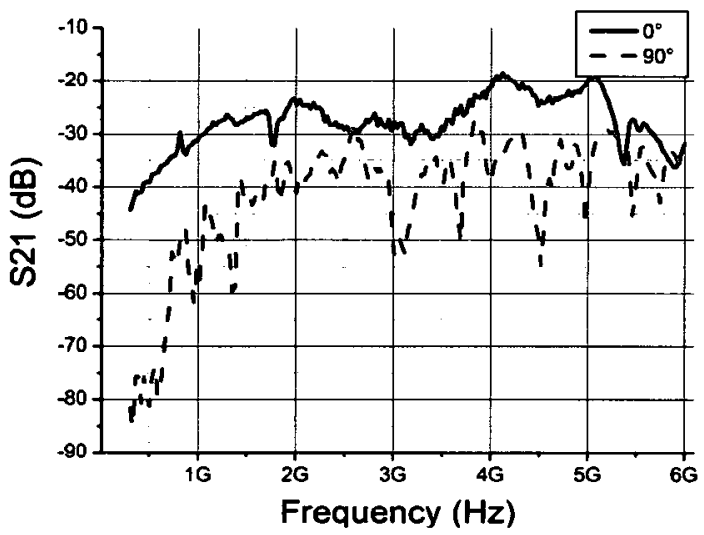

(b)

\section{Double cube probe}

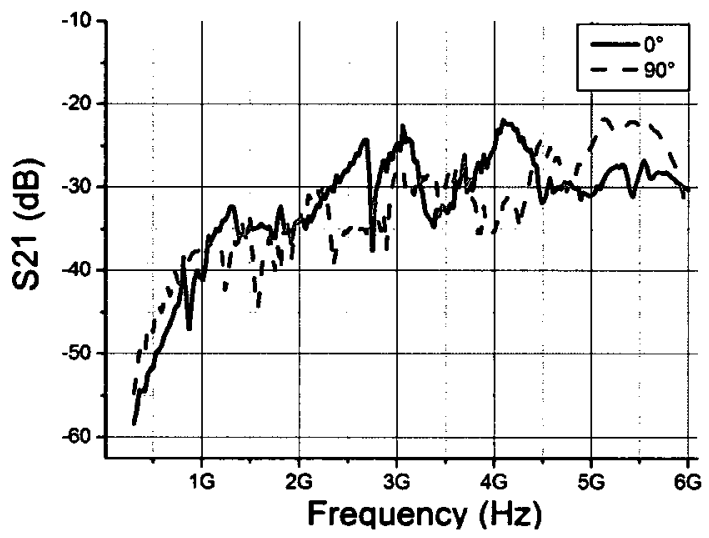

(d)

Figure 3-7: Sensitivity of probes in two orthogonal orientations: (a) cube probe, (b)

tangential probe, (c) normal probe, (d) and double cube probe.

All probes follow the general trend of increased sensitivity with frequency depicted on Figure 2-7; nonetheless each probe is seen to exhibit its own characteristics as shown on Figure 3-7. The coupling in the two orientations 
Study of Miniature Magnetic Field Probes

differs substantially for the tangential probe which is expected due to the linear

geometry of the EMI source. Also, as expected in this particular geometry, the normal probe's sensitivity has less variation between the two orientations. That being said, in the presence of more complex test structures, the sensitivity of the normal and tangential probe would drop since it can only sense magnetic fields in one plane at a time.

As desired, the coupling for both the cube probe and the double cube probe remains fairly constant for both orientations. Moreover, the double cube probe displays greater coupling predominantly at higher frequencies due to its higher magnetic flux density [7].

Based on the characterization results for the cube probe and double cube probe, it can be concluded that these structures are suitable integrated elements for near field EMC/EMI diagnosis [7].

\subsection{Cube Probe in LTCC}

Low Temperature co-fired ceramic (LTCC) technology has proven to be a great fit for high volume and low cost production of system on package [27]. At RF frequencies, LTCC is a high performance environment with relatively low losses that depend on the ceramic type and its firing time, and on the conductor material and its thickness [28]. For this reason, LTCC is becoming a very popular packaging option with its high frequency operation pushing to $100 \mathrm{GHz}$. It should be noted that LTCC offers a low temperature expansion coefficient and a 3D medium for embedded (L,C) passives having a high $Q$ [29]. Carleton University 
Study of Miniature Magnetic Field Probes 33 has developed many structures in LTCC ranging from antennas $[30,31]$ to miniature filters $[32,33]$ and full systems on package SoP [34].Therefore, the design process is well established within the Department of Electronics.

Thus, embedding the cube probe structure in LTCC is a reliable technology choice. Furthermore, the co-integration of the required active components to select and amplify the probe signals can be easily accomplished in LTCC.

\subsection{Characterization of the Cube Probe in LTCC}

The new cube probe structures developed in HDI were implemented in LTCC. To accurately characterize the cube probes, the structure was simulated in Ansoft HFSS, to determine its input impedance and its near field pick up of magnetic fields emitted from a microstrip transmission line. To validate the simulations, and to verify experimentally the behaviour of the probes, two cube probe structures were built to be tested by probing, and through a surface mount SMB connector. Figure 3-8 shows the cube probe structure fabricated in LTCC. 


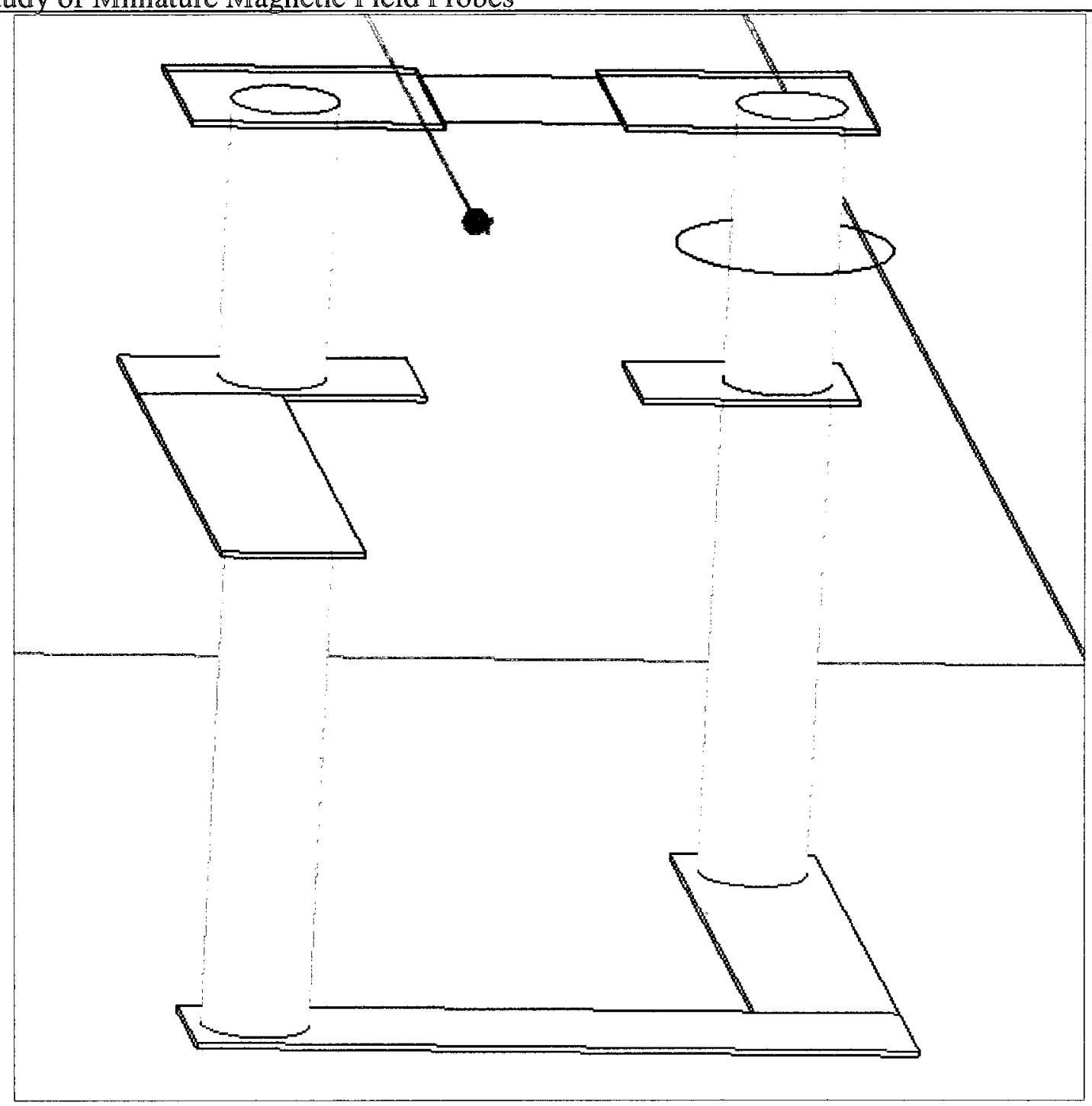

Figure 3-8: Cube Probe Structure in LTCC

\subsubsection{Simulated results in Ansoft HFSS}

To verify initially the behaviour of the cube probe in an LTCC substrate, the probe was simulated by placing a lumped port between both ends of the cube probe and by grounding one end. The simulations were performed between 0.1 $\mathrm{GHz}$ and $6 \mathrm{GHz}$. The magnitude and phase of its input impedance are shown in Figure 3-9. Then, through the use of Agilent-ADS, an electric model was found 
Study of Miniature Magnetic Field Probes

for the simulated cube probe to find its equivalent circuit. The model is necessary for comparison purposes to gauge the performance of the cube probe in LTCC with respect to the one realized in HDI. The derived electric model is shown in Figure 3-10. Other model topologies were tried but did not match as well as the one in Figure 3-10.

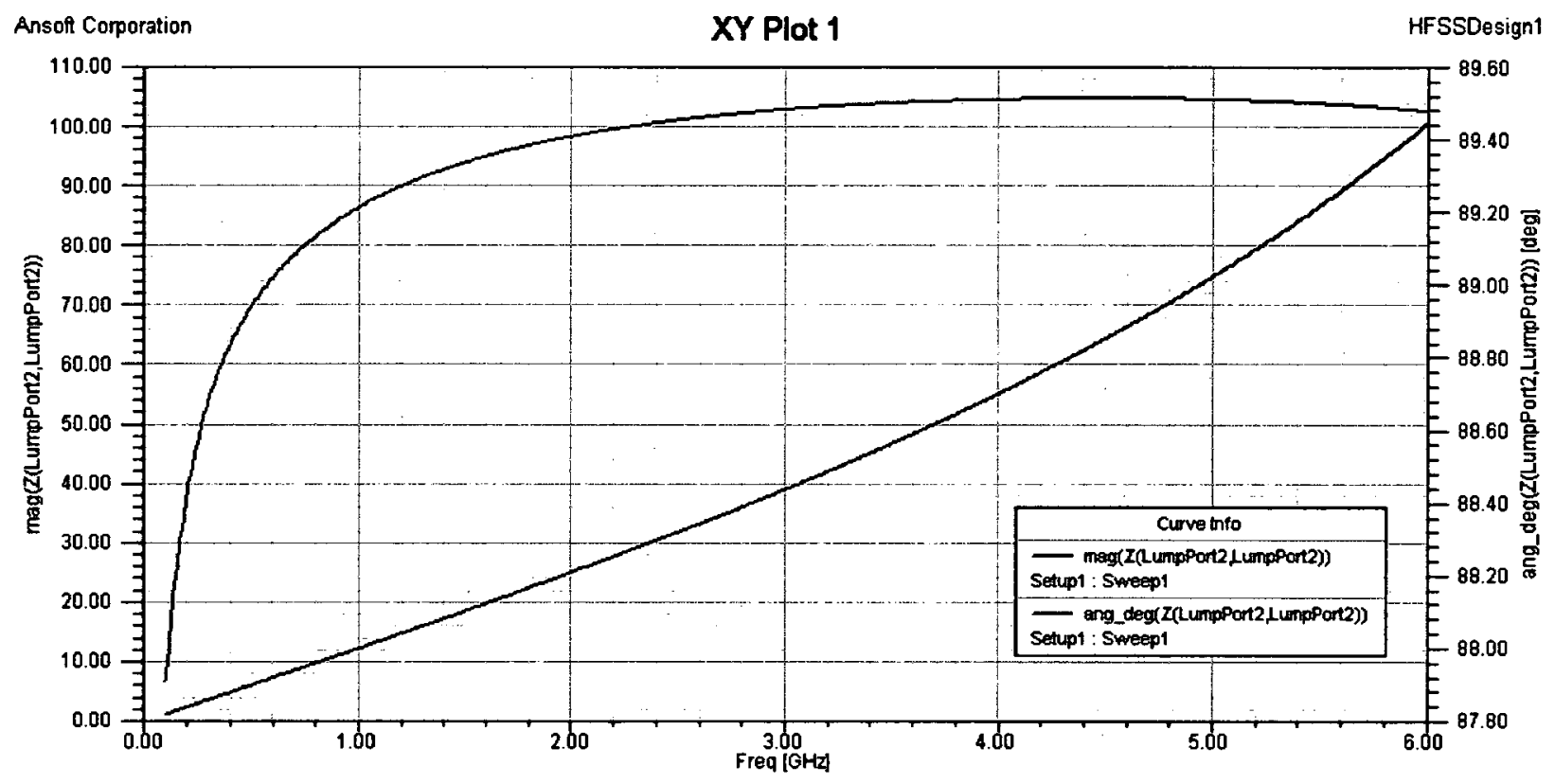

Figure 3-9: Magnitude and phase of the input impedance of the Cube Probe in LTCC 


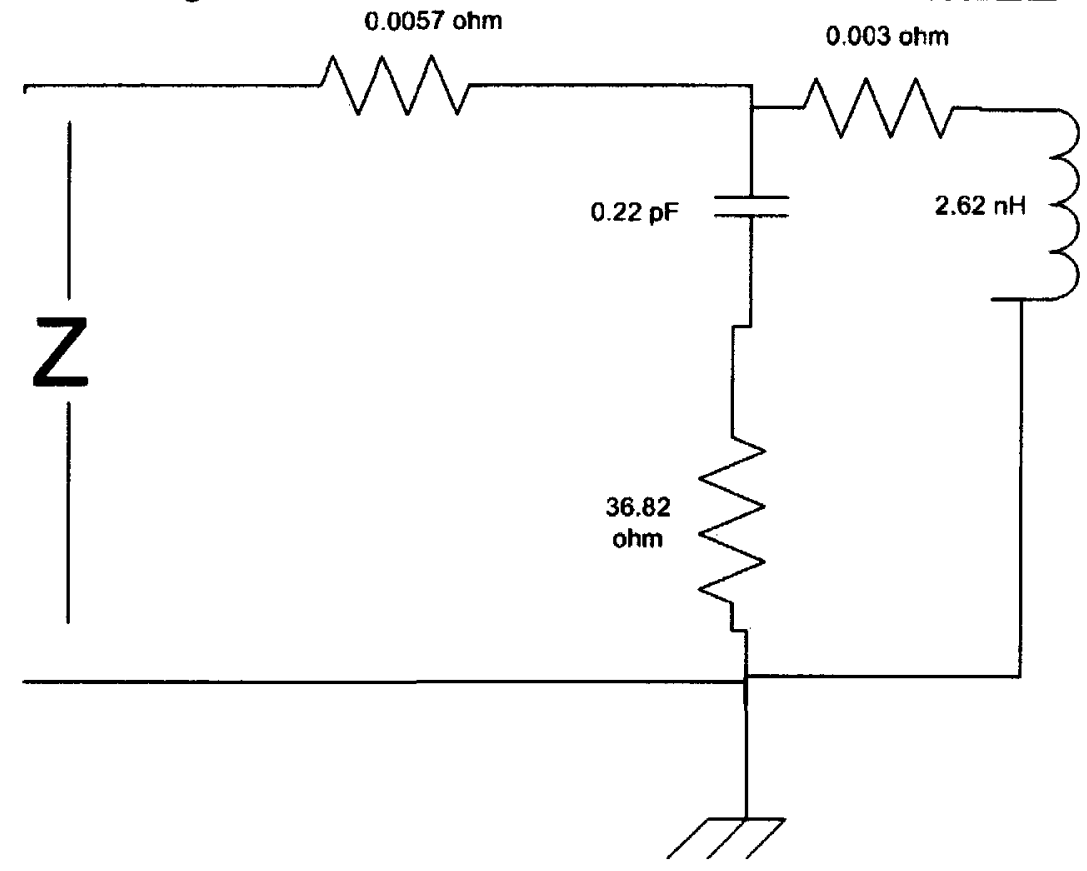

Figure 3-10: Electrical equivalent model of a cube probe simulated in Ansoft HFSS

\subsubsection{Fabrication of Cube Probe in LTCC}

To validate the simulations, a cube probe was built in LTCC where a GSG 500 $\mu \mathrm{m}$ pitch probe can be used to measure its input impedance. The fabrication was performed by VTT Electronics in Finland [35]. The characteristics of the LTCC are the following:

Number of Layers: 8

Permittivity: 5.9

Loss Tangent: 0.002

Some differences are present between the cube probe built in $\mathrm{HDI}$ and the one built in LTCC. Since the LTCC block is comprised of 8 layers, with a thickness of $0.1 \mathrm{~mm}$ per layer, the height of the cube probe in LTCC is set at $0.7 \mathrm{~mm}$ as 
Study of Miniature Magnetic Field Probes opposed to a height of $1 \mathrm{~mm}$ in HDI. Furthermore, the metal width in LTCC is of $0.2 \mathrm{~mm}$ as opposed to $0.1 \mathrm{~mm}$ in HDI. However, the vias' diameter is kept at 0.15 mm just as in HDI.

Furthermore, with the intent to characterize surface mount RF connectors, the same cube probe described above was also built in LTCC, with the same microwave properties mentioned above. The new LTCC module is formed of 19 layers and its fabrication procedure is described in detail in chapter 4 of this thesis. The ground and signal connections on the cube probe are extended to two pads on the surface of the LTCC module. A surface mount RF connector is placed in between both pads. The motivation behind this characterization is to validate the use of the connectors for future measurements.

\subsubsection{Results of Cube Probe "on-wafer" Measurements}

To avoid any effects from the metal chuck on which the LTCC substrate is placed, a block of foam is inserted between the two. The foam has a permittivity very close to that of air (around 1). The thickness of the foam, measured at $2 \mathrm{~cm}$, is sufficient to isolate the cube probe such that its input impedance is not altered by the surrounding metal. The measured input impedance (red) and the equivalent model's input impedance (blue) are both shown on Figure 3-11. It is shown that there is a good match between both curves until about $4 \mathrm{GHz}$ in frequency. The electrical equivalent model of the on-wafer cube probe can be seen on Figure 3-12. 


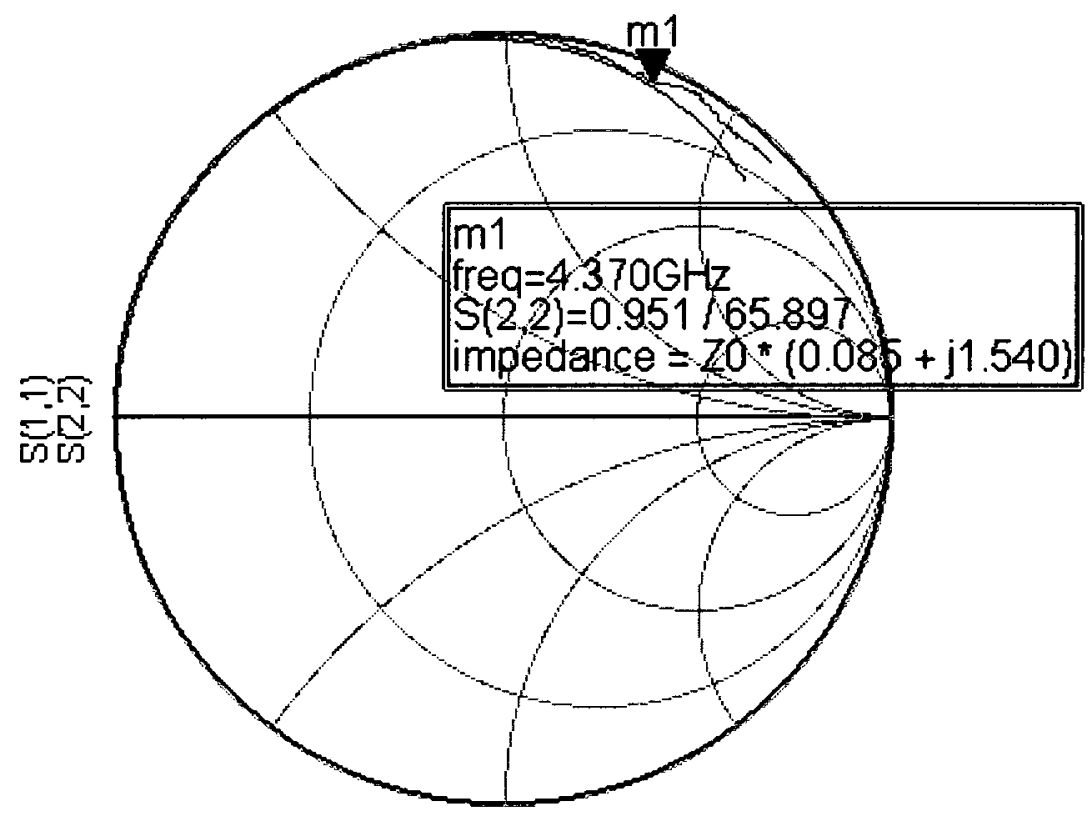

freq $(100.0 \mathrm{MHz}$ to $6.000 \mathrm{GHz})$

Figure 3-11: Input impedance of the Cube Probe measured "on-wafer" and of the equivalent model

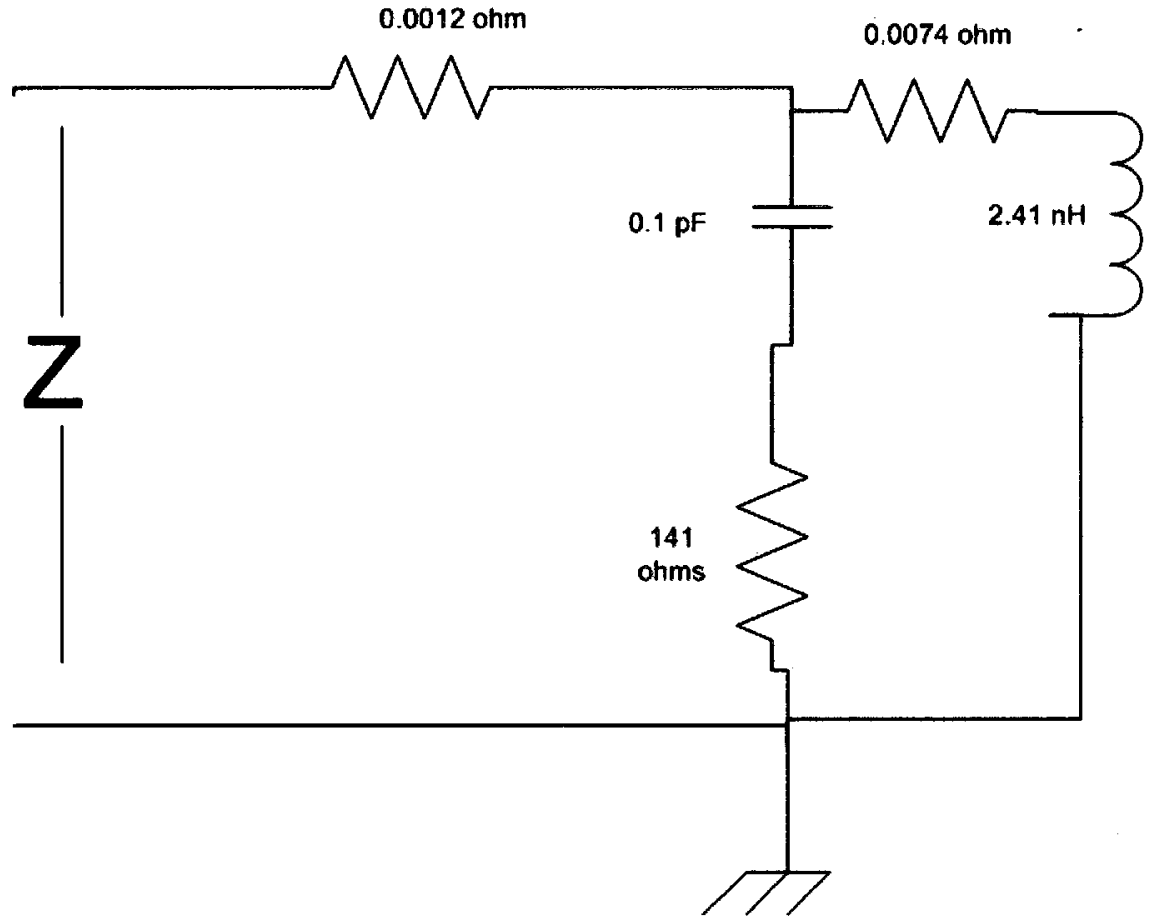

Figure 3-12: Equivalent model of the probed Cube Probe 
Study of Miniature Magnetic Field Probes

\subsubsection{Results of Cube Probe "connectorized" Measurements}

The input impedance of the cube probe was obtained via an RF surface mount

SMB connector. Figure 3-13 shows the measured input impedance response on the Smith Chart.

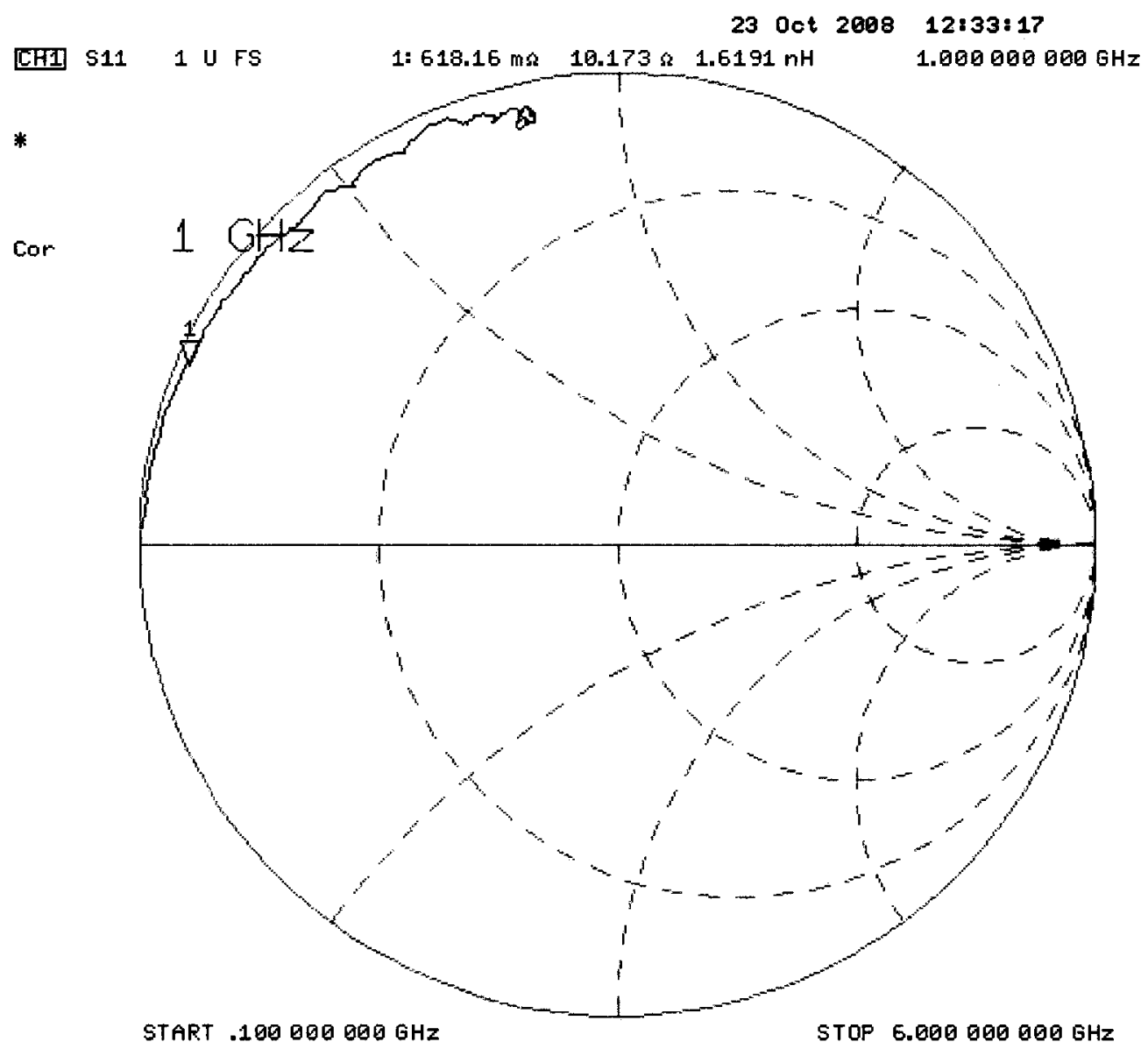

Figure 3-13: Input Impedance of the Cube Probe in LTCC

When comparing Figure $3-13$ with Figure $3-11$, it is found that the "connectorized" cube probe shows a shorter length of line compared to the "onwafer" cube probe. This result is unexpected given that the connector adds phase length to the measurement which is the opposite of what is observed in 
Study of Miniature Magnetic Field Probes 40

Figure 3-13. This result is due to calibration and is explained in the following section.

\section{De-embedding of the surface mount connector}

To be able to compare the performance of the "connectorized" cube probe with the "on-wafer" measurements and with simulations, a de-embedding process is required to remove the effect of the connector from the measurement results.

The surface mount connector employed is an RF SMB connector (\#1055687-1) from Tyco Electronics. The connector uses three ground pins and a centre conductor pin. However, the layout in LTCC was not optimized for the use of these connectors because the choice of connector was not made at the time of the LTCC fabrication. Thus, only one ground pin and the centre conductor pin were used. This connection scheme is known to introduce parasitic effects; however, given the limited frequency range $(<3 \mathrm{GHz})$, these effects were considered to be acceptably small.

To de-embed the effect of the connector on the measurements, a transmission line component is defined in HP-ADS that exhibits the same magnitude and phase changes as those of the surface mount connector. Such transmission line characteristics can be formed from a sample connector had its ground pin and centre conductor pin shorted. An S11 measurement of the shorted connector was performed and recorded as an S1P file. During the calibration process, since the calibration kit standards are only fit for SMA connectors, and additional SMB to SMA adapter was utilized. Therefore, the plane of reference of the S- 
Study of Miniature Magnetic Field Probes 41 parameter measurements was moved beyond the shorted connector. This change of reference planes made for the shorted connector to be represented by a physical transmission line named TLINP in HP-ADS, with a negative length of line. This line was found to have the same variation in magnitude and phase over the frequency range of operation. The transmission line had the following parameters:

- Length: $-4.2 \mathrm{~mm}$

- Permittivity: 2.2

- Loss Tangent: 0.002

- Impedance: 50 ohms

Following the de-embedding procedure found in [36], the input impedance to the probe itself was found and the cube probe's equivalent model was then produced. Figure 3-14 shows the equivalent model for the de-embedded probe. 


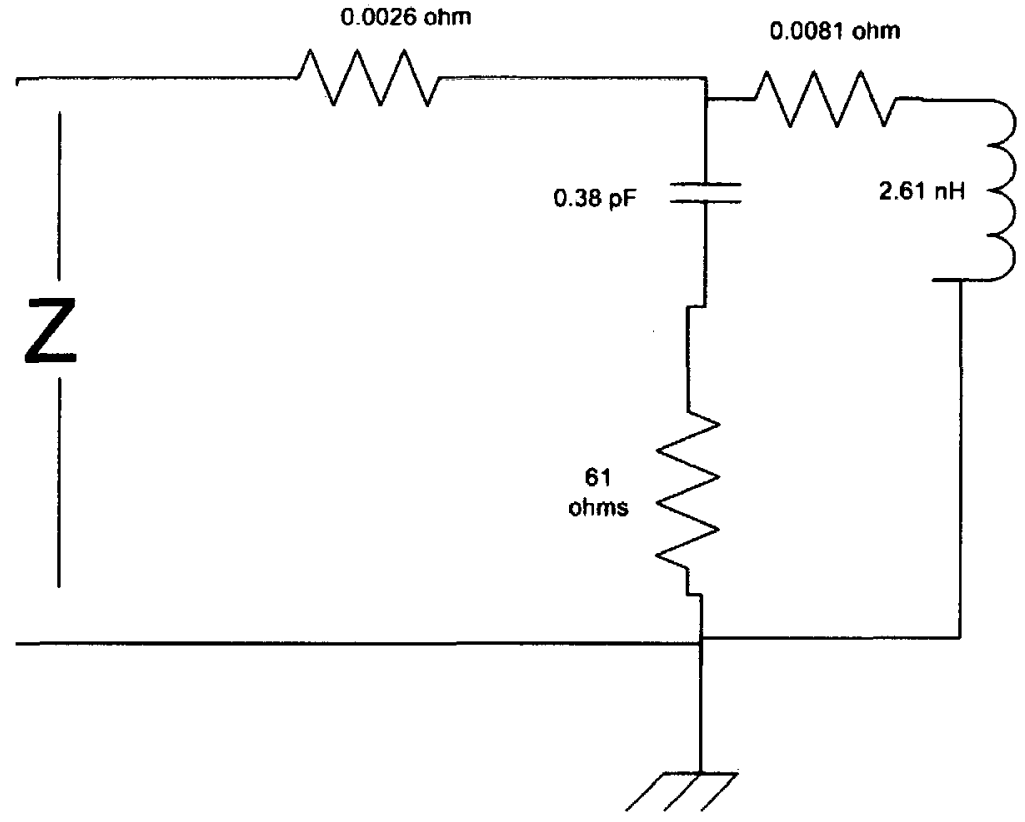

Figure 3-14: Equivalent model of the cube probe in LTCC

Table 3-1 serves as a comparison tool for all the equivalent models found for the cube probe in LTCC. The three models are derived from simulation, on-wafer measurement, and connectorized measurement of the input impedance.

The conclusions we can deduce from all these results are the following:

- The inductance of the cube probe in LTCC is around $2.5 \mathrm{nH}$

- The capacitance of the cube probe is around $0.2 \mathrm{pF}$

- A resistive element varying between 38 and 141 ohms.

\begin{tabular}{|l|c|c|c|c|}
\hline & $\mathrm{L}$ & $\mathrm{C}$ & $\mathrm{R}$ & Comments \\
\hline Simulated cube & $2.6 \mathrm{nH}$ & $0.22 \mathrm{pF}$ & $\begin{array}{c}38.62 \\
\text { ohms }\end{array}$ & \\
\hline On-wafer cube & $2.4 \mathrm{nH}$ & $0.1 \mathrm{pF}$ & $141 \mathrm{ohms}$ & $\begin{array}{c}\text { Small difference in inductance } \\
\text { and capacitance due to feed pad } \\
\text { geometry }\end{array}$ \\
\hline $\begin{array}{l}\text { Connectorized } \\
\text { cube }\end{array}$ & $2.6 \mathrm{nH}$ & $0.38 \mathrm{pF}$ & $61 \mathrm{ohms}$ & \\
\hline
\end{tabular}

Table 3-1: Comparative study of the input impedance of the cube probe in LTCC 
Study of Miniature Magnetic Field Probes

The electric models for the cube probe in LTCC show a consistent inductance of around $2.5 \mathrm{nH}$, as found through simulations and measurements. The measurements were performed through probing, which had the LTCC substrate set over a block of foam to minimize interference from a metal chuck. The input impedance of the cube probe was measured as well through a surface mount SMB connector. The effect of the connector was de-embedded, and the actual input impedance of the probe was obtained while it was suspended in the air.

Although the resistor values obtained in the models vary between the simulation and both measurements, these differences are explainable. The resistor is series with the capacitor represents the $Q$ of the parasitic capacitor and the loss existing in the substrate. In simulation, the permittivity of LTCC and the loss tangent are set as an estimate of the real value. Therefore, it is apparent that the loss tangent estimate is optimistic. To study the real effect of the resistor value in the equivalent model, the resistor value was varied in simulation. The effect of the resistor variation is observed at the higher frequencies (beyond $3 \mathrm{GHz}$ ) when significant power is being shorted through the capacitor. Therefore, in the presence of noise at high frequencies, specially in the case of the connectorized measurement, the value of the resistance can be significantly altered in the equivalent model.

The differences observed in the models are minor, and the change in the resistor values produce minor changes on the smith chart. Therefore, we can deduce the following conclusions: 
Study of Miniature Magnetic Field Probes 44

- Three ways to find the input impedance of the cube probe yielded similar results.

- The simulation results produced by Ansoft HFSS were validated and are trustworthy.

- The de-embedding results of the measurements with the surface mount connector are accurate.

\subsubsection{Sensitivity of cube probe to circuit level EMI}

Although the equivalent model gives insight into the possible mutual inductive coupling between the cube probe and an adjacent structure (and therefore into the probe's sensitivity), it is always important to directly observe the operation of the probe by measuring its EMI pick up. To accomplish this, a cube probe was placed $1.1 \mathrm{~mm}$ above a $50 \mathrm{ohm}$ microstrip line (width of $5 \mathrm{~mm}$, on a Rogers Duroid 5880 board) terminated by matched load of 50 ohms as was done in section 3.2. The input port of the transmission line was connected to port 1 of a network analyzer and the output of the cube probe was connected to port 2. A measured $\mathrm{S}_{21}$ over $0.1 \mathrm{GHz}$ to $6 \mathrm{GHz}$ frequency range showed the probe's pick up with respect to frequency. The apparatus and the testing procedures are identical to those used in [7]. Figure $3-15$ shows the measured results of the LTCC cube probe's pick up. 


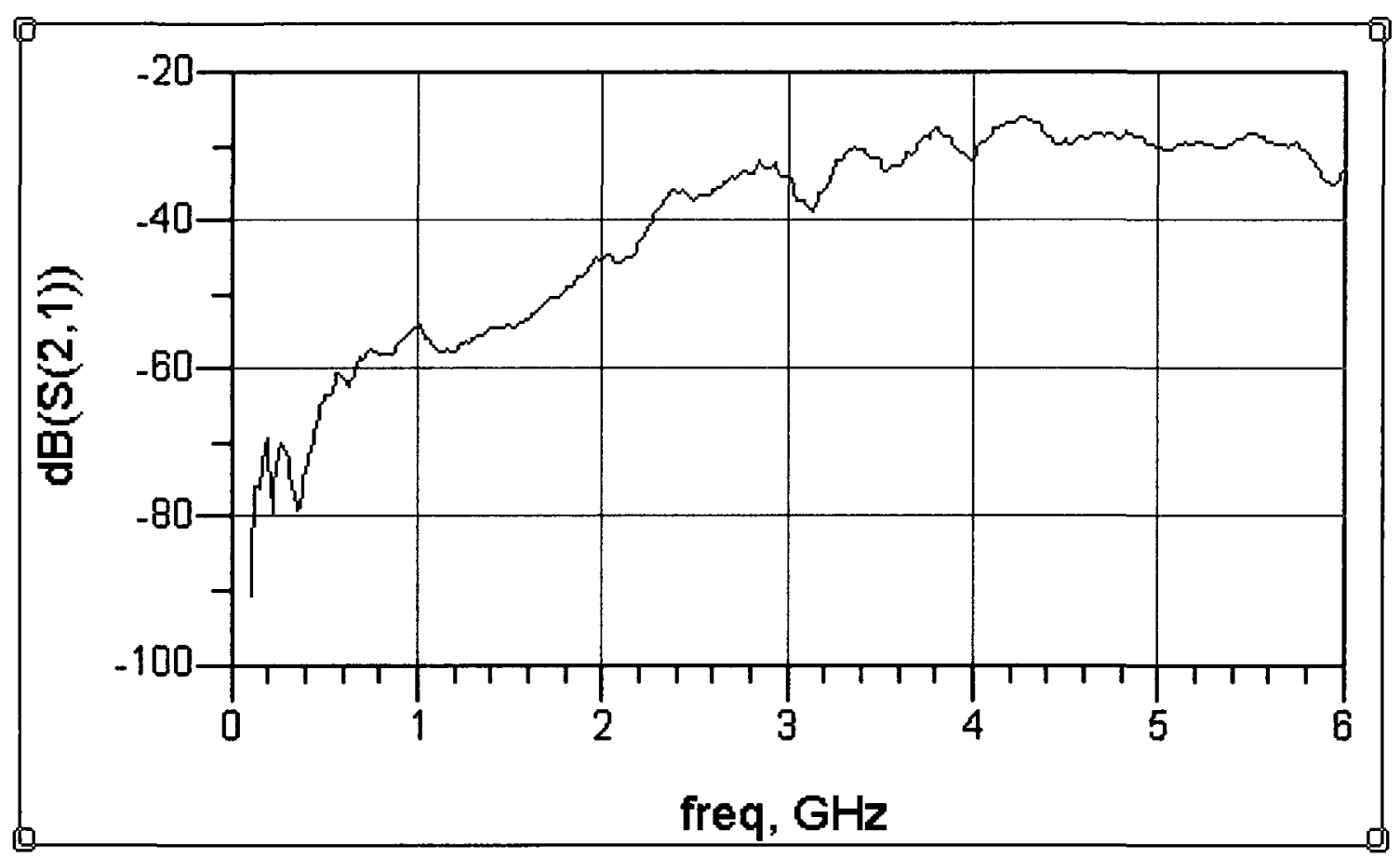

Figure 3-15: Cube Probe in LTCC magnetic field pick up with respect to frequency By comparing Figures $3-15$ and $3-7 a$, it is clear that the probe in $\mathrm{HDI}$ is more sensitive in the lower frequencies of operation. When inspecting the equivalent models of both cube probes, it is evident that the cube probe in HDI possesses a higher inductance value. This change of inductance value is due to the geometry changes in the cube probe as described in section 3.5.2. The cube probe in LTCC is smaller in the height dimension $(0.7 \mathrm{~mm}$ versus $1 \mathrm{~mm})$ and it possesses thicker metal lines $(0.2 \mathrm{~mm}$ versus $0.1 \mathrm{~mm})$ both of which reduce the inductance comparatively. At higher frequencies, the sensitivity of both probes seem to match more closely. The closeness in the match can be explained by the lossier 
Study of Miniature Magnetic Field Probes 46 environment in $\mathrm{HDI}$ at higher frequencies, which is represented by the higher resistance value in the equivalent model for the HDI probe.

\subsubsection{Double Cube Probe in LTCC}

The double cube probe, introduced in section 3.2, presents an alternative design for a magnetic near field antenna, with an enhanced energy pick up due to its higher inductance.

The LTCC double cube probe was also measured "on-wafer" in the same manner as for the simple cube probe. The LTCC substrate was placed above a layer of foam to remove any effects produced by the station's metal chuck. Figure 3-16 shows the input impedance result, and the resulting equivalent model is shown on Figure 3-17.

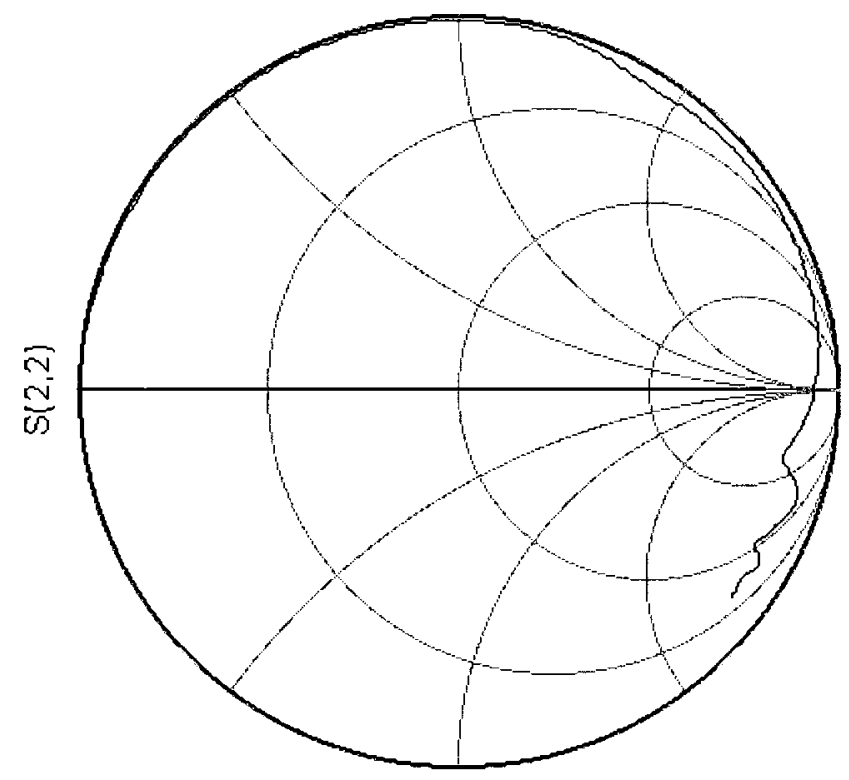

freq $(100.0 \mathrm{MHz}$ to $6.000 \mathrm{GHz})$

Figure 3-16: input impedance of the double cube probe measured "on-wafer" 


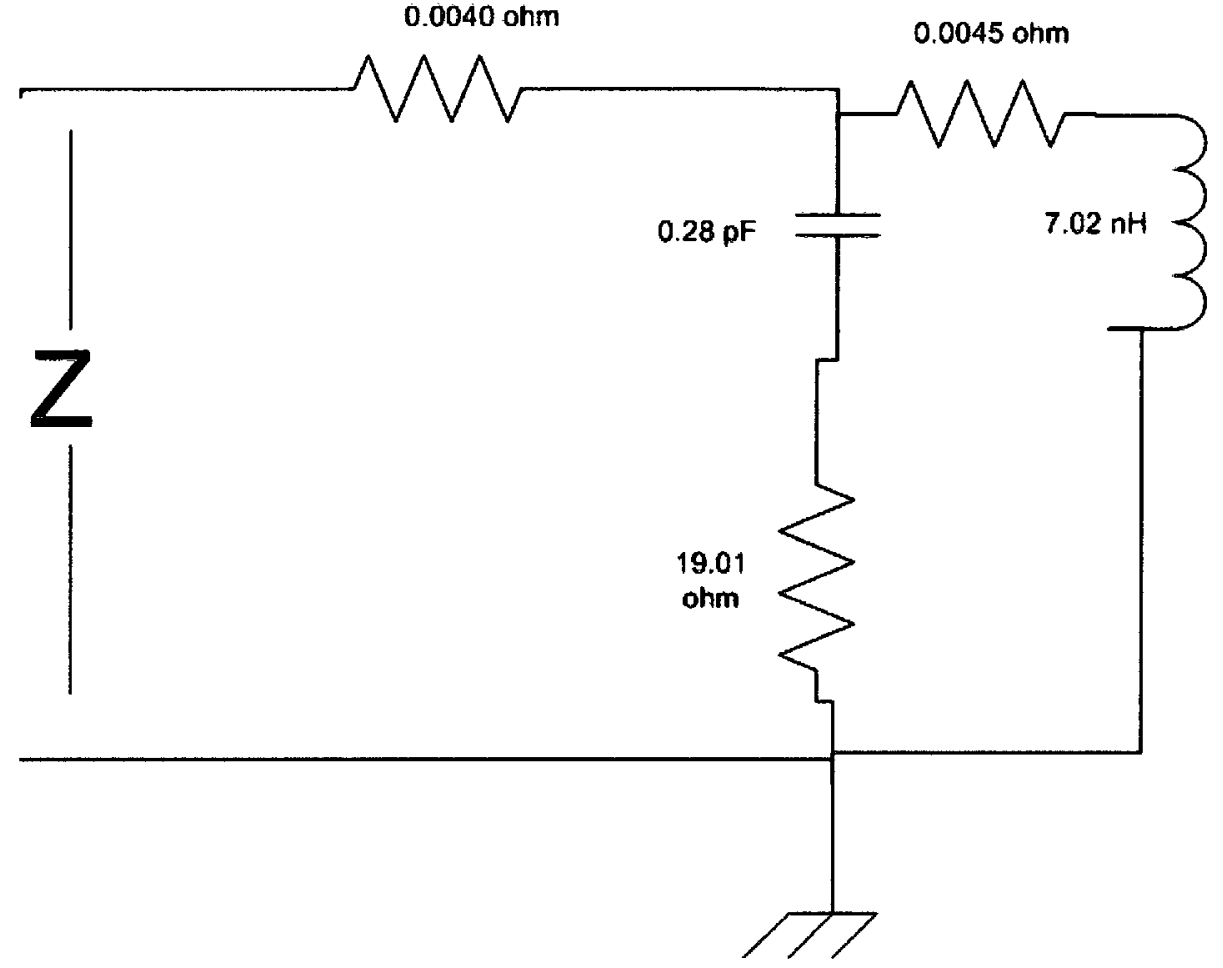

Figure 3-17: Equivalent electrical model for a Double Cube Probe in LTCC

To increase the sensitivity of the cube probe, the double cube probe presents a very nice alternative. The inductance achieved with the double cube probe is around $7 \mathrm{nH}$. This enhanced probe serves as a good option for low frequency detection of EMI, given that the increased inductance and the increased parasitic capacitance present in its equivalent model creates a lower resonant frequency.

\subsection{Cube Probe in Ferrite LTCC}

Ferrite tapes have been recently added to the LTCC material system to form ferrite LTCC modules [37]. This composite material has an increased permeability, which could help EMI detection by inducing a higher mutual coupling at the cube probe level. Two ferrite LTCC tapes were previously tested 
Study of Miniature Magnetic Field Probes

by members of the group supervised by Professor Langis Roy at Carleton University. As with any ferrite material, application of a static (DC) magnetic bias will change its permeability tensor. The first tape was donated by Ferro Corporation and the second was the ESL 40012 ferrite LTCC. The ESL 40012 achieved higher magnetisation saturation by comparison with the Ferro Corporation tape [37].

In order to assess the merits of ferrite LTCC for EMI characterization, cube probes were studied using the ESL 40012 tape. In the unbiased states, the ferrite LTCC can achieve a permeability of 4 depending on the frequency of operation in the 0.1 to $2 \mathrm{GHz}$ range. When the ferrite LTCC is biased with DC current, through a winding, the achieved permeability can reach 370 . [37]

The drawback with this material is that between $2 \mathrm{GHz}$ and $9 \mathrm{GHz}$, the low field losses associated with the domain wall rotation resonances can increase the microwave losses dramatically in the unbiased state.

The ferrite cube probe, having the same dimensions as the previous LTCC design, was simulated in Ansoft HFSS. The following LTCC material properties were employed:

- Permittivity: 13.6

- Loss tangent: 0.002

- Permeability: 4

The simulations were performed between $100 \mathrm{MHz}$ and $1 \mathrm{GHz}$. The equivalent model is deduced from the input impedance of the ferrite cube probe and is 
presented on Figure 3-18. The model presents a much higher inductance than the models for the Cube Probe in regular LTCC which was expected. The difference in inductance is significant as it jumps from around $2.5 \mathrm{nH}$ to around 7 $\mathrm{nH}$. Thus, the ferrite cube probe presents a highly sensitive EMI sensor at low frequencies

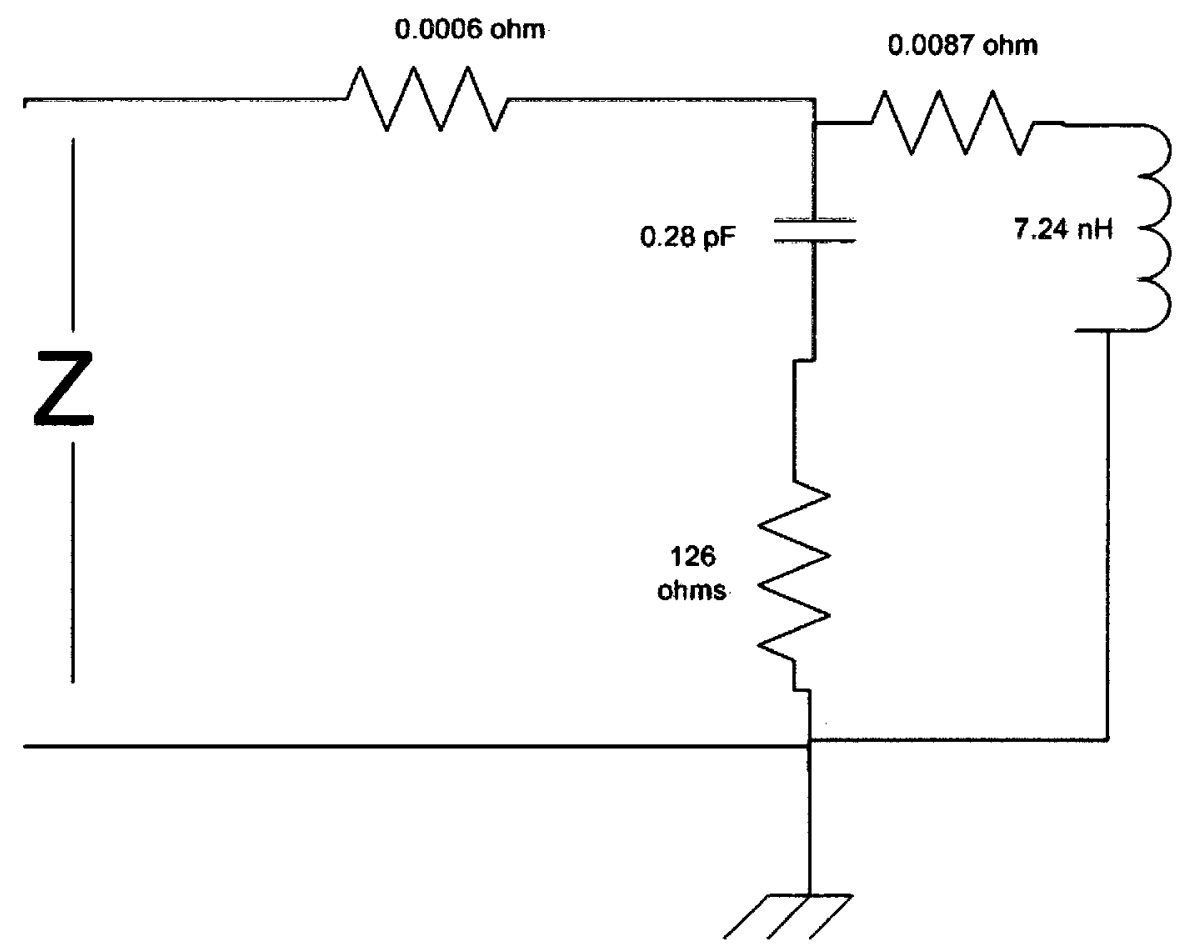

Figure 3-18: Equivalent model of the ferrite LTCC cube probe

As mentioned above, the ferrite cube probes are only meant to be used at low frequencies in their unbiased state due to the low field losses. On the other hand, in the biased state, the low field losses will disappear, paving the way for a much higher inductance and a much greater sensitivity at higher frequencies. 


\subsection{Summary}

Chapter 3 discussed in detail the cube probe in three material systems. An analysis, accompanied by simulations and measurements, is presented for the cube probe and the double cube probe. The findings of chapter 3 are tabulated in Table 3-2. This table serves as a comparison between the cube probe structures across three material systems: HDI, LTCC, and ferrite LTCC.

\begin{tabular}{|l|c|c|c|c|c|}
\hline & $\mathrm{L}$ & $\mathrm{C}$ & $\mathrm{R}$ & $\begin{array}{c}\text { Sensitivity } \\
(1 \mathrm{GHz})\end{array}$ & $\begin{array}{c}\text { Sensitivity } \\
(3 \mathrm{GHz})\end{array}$ \\
\hline HDI simple & $5 \mathrm{nH}$ & $0.15 \mathrm{pF}$ & 250 ohms & $-35 \mathrm{~dB}$ & $-35 \mathrm{~dB}$ \\
\hline LTCC & $2.5 \mathrm{nH}$ & $0.2 \mathrm{pF}$ & 70 ohms & $-55 \mathrm{~dB}$ & $-35 \mathrm{~dB}$ \\
\hline HDI Double & $10 \mathrm{nH}$ & $0.5 \mathrm{pF}$ & 750 ohms & $-35 \mathrm{~dB}$ & $-25 \mathrm{~dB}$ \\
\hline $\begin{array}{l}\text { LTCC } \\
\text { Double }\end{array}$ & $7 \mathrm{nH}$ & $0.28 \mathrm{pF}$ & 19 ohms & - & - \\
\hline $\begin{array}{l}\text { Ferrite } \\
\text { LTCC }\end{array}$ & $7.2 \mathrm{nH}$ & $0.28 \mathrm{pF}$ & 126 ohms & - & - \\
simple & & & & & \\
\hline
\end{tabular}

Table 3-2: Comparison between the cube probes studied in chapter 3

Based on the above result, it is clear that the inductance found in HDI is higher and that is due to its geometry. By comparison to the probes in LTCC, the probes in $\mathrm{HDI}$ possess an increased height parameter ( $1 \mathrm{~mm}$ as opposed to $0.7 \mathrm{~mm}$ ) and thinner metal lines, all contributing to higher inductance. Therefore, it is not 
Study of Miniature Magnetic Field Probes surprising that the cube probe in $\mathrm{HDI}$ is more sensitive to EMI than the cube probe in LTCC at around $1 \mathrm{GHz}$. However, it is noticeable that the cube probe in LTCC is able to achieve the same sensitivity than the cube probe in HDI at 3 $\mathrm{GHz}$. This is due to the lower microwave losses in LTCC at higher frequencies explained by the lower resistances found for the models of the cube probe in LTCC.

On the other hand, the ferrite LTCC cube probe presents a very high inductance in the unbiased state, able to increase drastically when the ferrite is biased; hence increasing the cube probe's sensitivity further. 


\section{Chapter 4}

\section{Development of EMI Characterization SoP}

As mentioned in chapter 2, the traditional way of testing EMI has required the usage of a magnetic or electric field probe displaced by a scan table over a device under test DUT. However, this method is time consuming and requires having a scan table with three different motors that can displace a probe in all three dimensions. The table size is big, and it requires a dedicated technician who is able to operate the software associated with its control. For smaller corporations, owning a system of the sort and making it operational can be expensive, which eliminates the possibility of performing EMC/EMI testing on their underdeveloped prototypes. A novel LTCC based system on package SoP can effectively replace the operation of the scan table by incorporating an array of cube probes. This chapter provides an overview of SoP technology, the custom multiplexer chip design, and finally the LTCC module development.

The created system paves the way for fast EMI measurements, as electrical steering using the multiplexer chips is a lot faster than the mechanical steering done by the scan tables. In addition, the system presents a handheld inexpensive solution that can be handy in any laboratory or working environment. The direct result of having a similar inexpensive system is the encouragement of all engineers to perform EMI measurements on their designs that wouldn't be normally done for lack of scan tables or money. 


\subsection{Overview of SoP Technology}

The development of Systems on Package SoP has emerged as a new technique in the 1990s, with much of the early work being led by the Georgia Institute of Technology. The purpose of this technique is to combine the elements of an electronics or mixed-technology system in one module, providing densely packaged solutions, and removing the need for bulky boards. Figure 4-1 shows the evolution of systems integration in packages as given by Tummala et al [38]. The definitions of the acronyms in Figure 4-1 are the following:

- MCM, multi-chip module: A 2D system that is able to contain various integrated circuits and their interconnects.

- SiP, System in Package: A 3D system in which the package is only used for the interconnects of integrated circuits.

- SoP, System on Package: A 3D system in which the package contains functional circuit elements (resistors, inductors, capacitors, antennas,...) in addition to the interconnects between various integrated circuits.

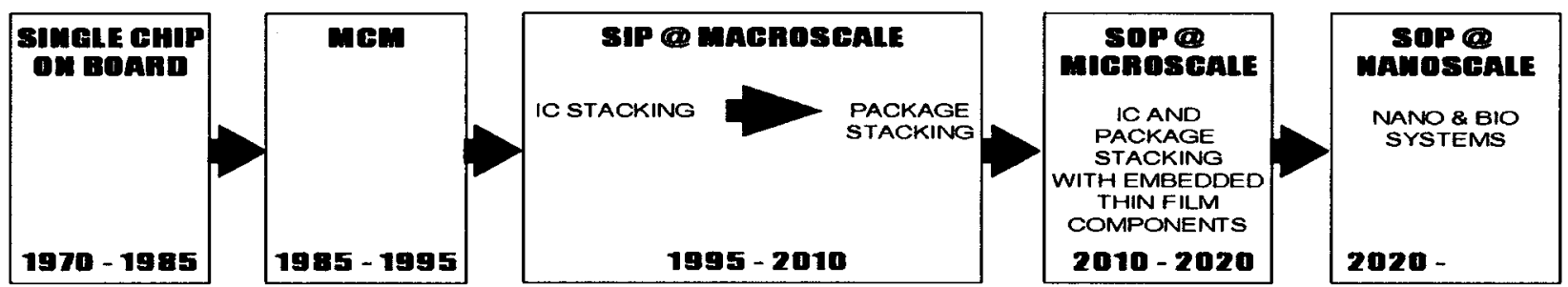

Figure 4-1: Evolution of packaging of systems [38]. 
Development of EMI Characterization SoP 54 With the ability of integrating chips, sensors and thin films, the co-designing of active and passive elements of a system is possible in a SoP [39]. With SoP techniques, the wire delay problem is drastically reduced. This is accomplished by the elimination of long connection wires and their replacement with slow global interconnects with short routes [40]. Thus, all the industries, ranging from wireless devices to biomedical systems, have benefitted from the advancements in integration [38].

A typical SoP may be a few centimetres per side, and carrying up to four different ICs for various analog, digital and optical functions [39]. The progress in photolithography techniques has allowed for very well defined conductor lines on the order of 5 to $10 \mu \mathrm{m}$, allowing for higher integration within a package [39].

While the most recent research indicates that Liquid Crystalline Polymer (LCP) based substrates are the prime candidates for SoP technology, LTCC is still a viable technology for SoP. The drawback with LTCC is its lack of compatibility with PCB material such as FR-4, due to its different coefficient of thermal expansion CTE. However, LTCC is still a good medium to embed high-Q passives [41]. LCP suffers from highly anisotropic properties, and is considered very expensive.

Based on the above, LTCC was employed as the technology of choice, due to the previous acquired experience in the group as mentioned in chapter 3 . Furthermore, the expertise at VTT Electronics in Finland, where the LTCC modules are fabricated, adds to the reliability of the final product [35]. 


\subsection{Design of LTCC-based SoP for EMI Detection}

\subsubsection{System Elements and Functions}

To fully replace the operation of a scan table, the newly developed cube probe structures are to be integrated in an array within the SoP module. An array of 64 probe elements was targeted with the elements spaced by $1 \mathrm{~mm}$ on each side (i.e. elements placed on a $2 \mathrm{~mm}$ grid). Each array element is responsible for measuring EMI at a given point above a DUT, effectively replacing the need to have a suspended magnetic field probe shifted by $1 \mathrm{~mm}$ with a scan table. To record the reading from each cube probe, a system of nine $8: 1$ multiplexers is chosen that uses 6 digital inputs. The combination of the 6 digital bits controls which cube probe's signal is to be fed to the output. Figure 4-2a shows the role of each multiplexer, passing the output of one cube probe from a sub-array of eight elements. Figure $4-2 b$ shows the total system with 64 cube probes and 9 multiplexers, where the outputs of the 8 peripheral multiplexers (red squares) are connected to the middle multiplexer.

Some attempts were made to find "off the shelf" multiplexer chips, however no 8:1 multiplexers were found that are able to propagate high frequency signals. 

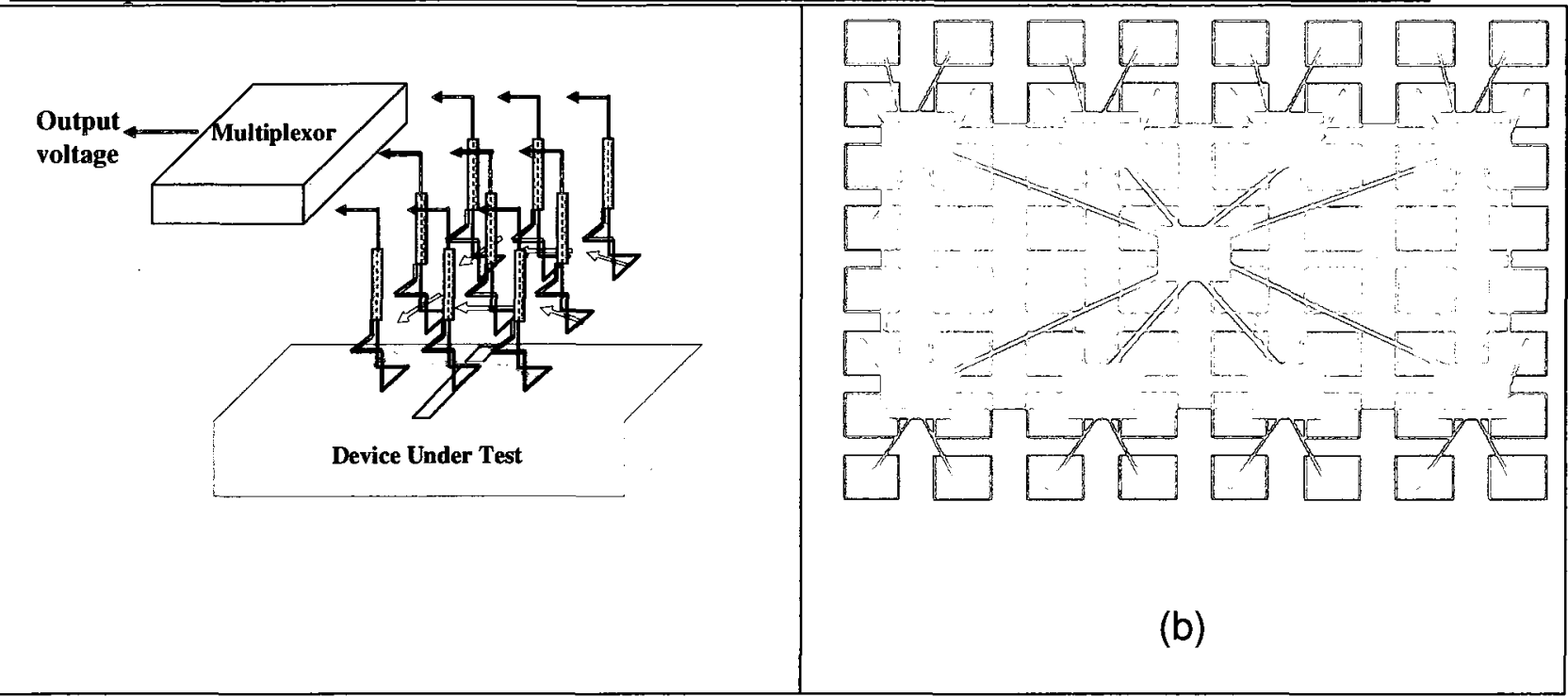

Figure 4-2: Graphical representation of the system in the SoP. a) Each 8 cube probes are connected to one multiplexer. b) All the outputs of the multiplexers are connected to a $9^{\text {th }}$ multiplexer for final selection.

Three different tasks are required for the development of this SoP. The first task was the design of a multiplexer chip, capable of taking eight RF inputs and passing one of them to the output while blocking the others. The second task is to design a custom LTCC package that houses the cube probes, the multiplexer chips, and their inter-connects. Finally, integrating the chips in the package and performing the $\mathrm{l} / \mathrm{O}$ assembly is the last task before testing and verifying the concept.

\subsubsection{Development of the multiplexer chip}

Typical multiplexer designs are valued by their ability to switch fast and to pass several Gb/s. With the improvement of lithography in CMOS processes, and the miniaturisation of the gate lengths, the maximum operating frequency has 
Development of EMI Characterization SoP 57 increased to tens of $\mathrm{GHz}$, making CMOS suitable for high speed, and high gain circuits [42]. Some of the recent advances in multiplexer designs in CMOS technology include the designing of a fully differential $8: 1$ current mode multiplexer. The advantage of such a multiplexer is in multiplexing in the current domain, allowing for large current swings but very low voltage swings, therefore charging and discharging capacitors rapidly [42].

Current mode logic (CML) was also used in [43]. A design of a 2:1 multiplexer using CMOS technology and $1 \mathrm{~V}$ of supply voltage was achieved, reaching data rates of $30 \mathrm{~Gb} / \mathrm{s}$. The clock signal is coupled through an on chip monolithic transformer making an efficient use of the total voltage swing available [43].

While most designers of multiplexers are strictly concerned with the switching rate they can support, the same concerns were not present for the multiplexer chip designed in this work. Since the control inputs to the multiplexer chips are relatively slow (coming from a programmed equipment interface), the speed requirement is not very stringent. The target of the design was to enable the transmission of EMI signals with frequencies ranging from $100 \mathrm{MHz}$ to $3 \mathrm{GHz}$, while minimizing the transmission losses. The switching is to occur at very low speeds, on the order of a few $\mathrm{Hz}$. With the availability of the $0.13 \mu \mathrm{m} \mathrm{CMOS}$ technology from IBM, meeting the bandwidth requirement is not an issue given the unity gain frequency $F_{t}$, on the order of $60 \mathrm{GHz}$ for this process. 


\section{Design of the multiplexer chip}

Based on the above specifications, the simplest design that would fulfill the requirements would be based on CMOS transmission gates [44]. The transmission gates would be followed by an output buffer that is capable of driving a 50 ohm load.

In its simplest form, a transmission gate is composed of two pass gates as shown on Figure 4-3 (a). The principal operation of the transmission gate is to allow the input signal to flow to the output when the selection bit $S$ is high, and to block the input from reaching the output when the selection bit $S$ is low. Figure 4-

3 (b) shows the equivalent model of a transmission gate. Based on the model, we can deduce that a transmission gate acts like a low pass filter. The following equations describe the model elements as found in [44].

The capacitances found in the model can be calculated as

$$
C_{o x}=\frac{\varepsilon_{o x}}{T_{o x}} W \cdot L
$$

Where $\varepsilon_{\text {ox }}$ is the permittivity of the oxide, $T_{\text {ox }}$ is the oxide thickness and $W$ and $L$ are the width and length of the channel respectively.

The channel resistance, defined as $R_{d s}$ in the equivalent model, can be estimated

$$
R_{d s}=R_{s h} \frac{L}{W}
$$

The symbol $R_{s h}$ in equation (4-2) represents the sheet resistivity in the MOSFET channel. Based on Equations (4-1) and (4-2), we can estimate the $3 \mathrm{~dB}$ cut-off frequency $f_{3 \mathrm{~dB}}$ as given by Equation (4-3). It can be seen that the cut-off frequency is proportional to the length of the devices utilized. On the other hand, 
Development of EMI Characterization SoP

an increased length increases the resistance of operation and produces more losses as given by equation (4-2).

$$
f_{3 d B}=\frac{1}{R C} \propto L^{2}
$$

Where $\mathrm{R}$ is formed of both channel resistances for the NMOS and PMOS

transistors in parallel, and $\mathrm{C}$ is the capacitance present between the output node in the model in Figure 4-3 (b) to ground.

The voltage transfer function of the transmission gate can be determined based on the produced model in Figure 4-3 (b) and is given by:

$$
\frac{V_{\text {out }}}{V_{\text {in }}}=\frac{Z_{C}}{Z_{C}+\left(R_{d s(N M O S)} / / R_{d s(\text { PMOS })}\right)}
$$

The impedance associated with the capacitance present in the model is given by $Z_{c}$, where:

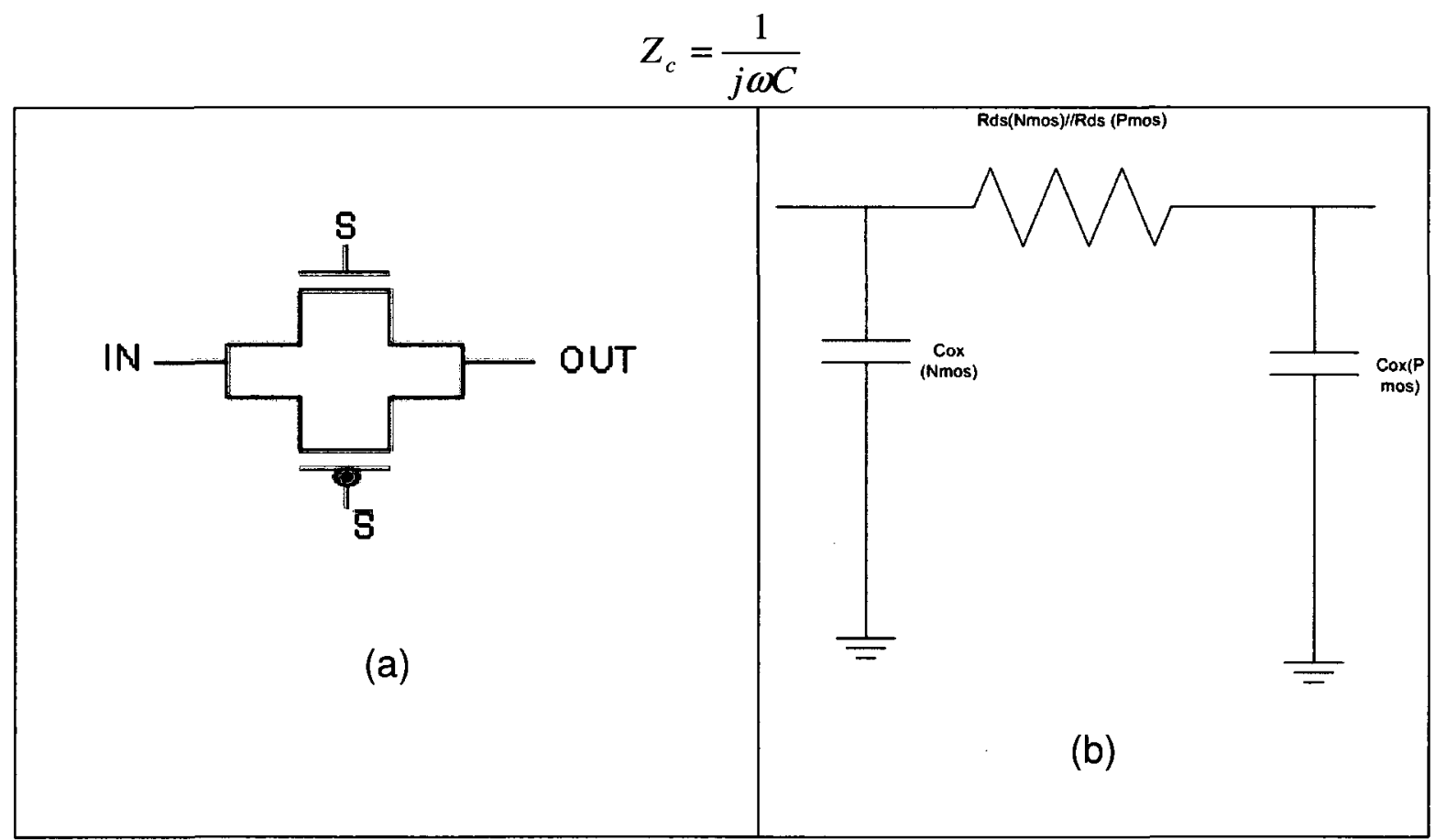

Figure 4-3: a) transmission gate, b) equivalent model of a transmission gate

The selection of passing a signal or blocking it using a transmission gate can be applied to a circuit with two inputs, thereby forming a 2:1 multiplexer. Based on 
Development of EMI Characterization SoP

60

the selection bit $S$, one of both inputs is passed to the output. Figure 4-4 shows the circuit of a 2:1 multiplexer.

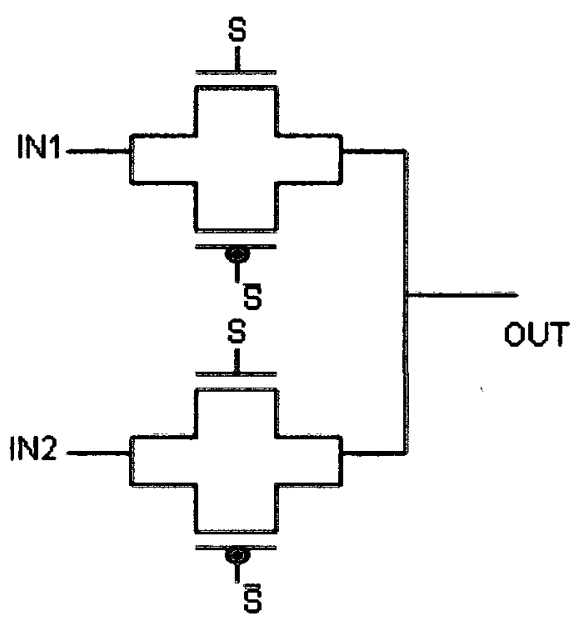

Figure 4-4: Circuit topology for a 2:1 multiplexer

To obtain some gain out of an output buffer, a common source topology can be used. In its simplest form, the common source topology is given on Figure 4-5. The voltage gain associated with a common source amplifier is given by:

$$
\frac{V_{\text {out }}}{V_{\text {in }}}=-g_{m} R_{\text {out }}
$$

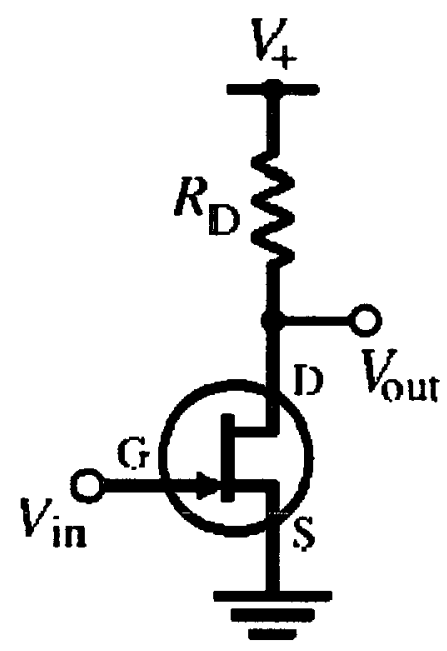

Figure 4-5: Simple common source amplifier 
Development of EMI Characterization SoP

With the available $0.5 \mathrm{~mm}^{2}$ allocated by $\mathrm{CMC}$, the multiplexer chip could only carry eight RF inputs. To switch between all the inputs, three digital bits are required. A chip enable is also introduced, done by inserting an extra pass gate to enable or disable a chip. Figure 4-6 shows the various building blocks that form the multiplexer chip. Figure 4-7 shows the circuit schematic of the 4-to-1 multiplexer implementation.

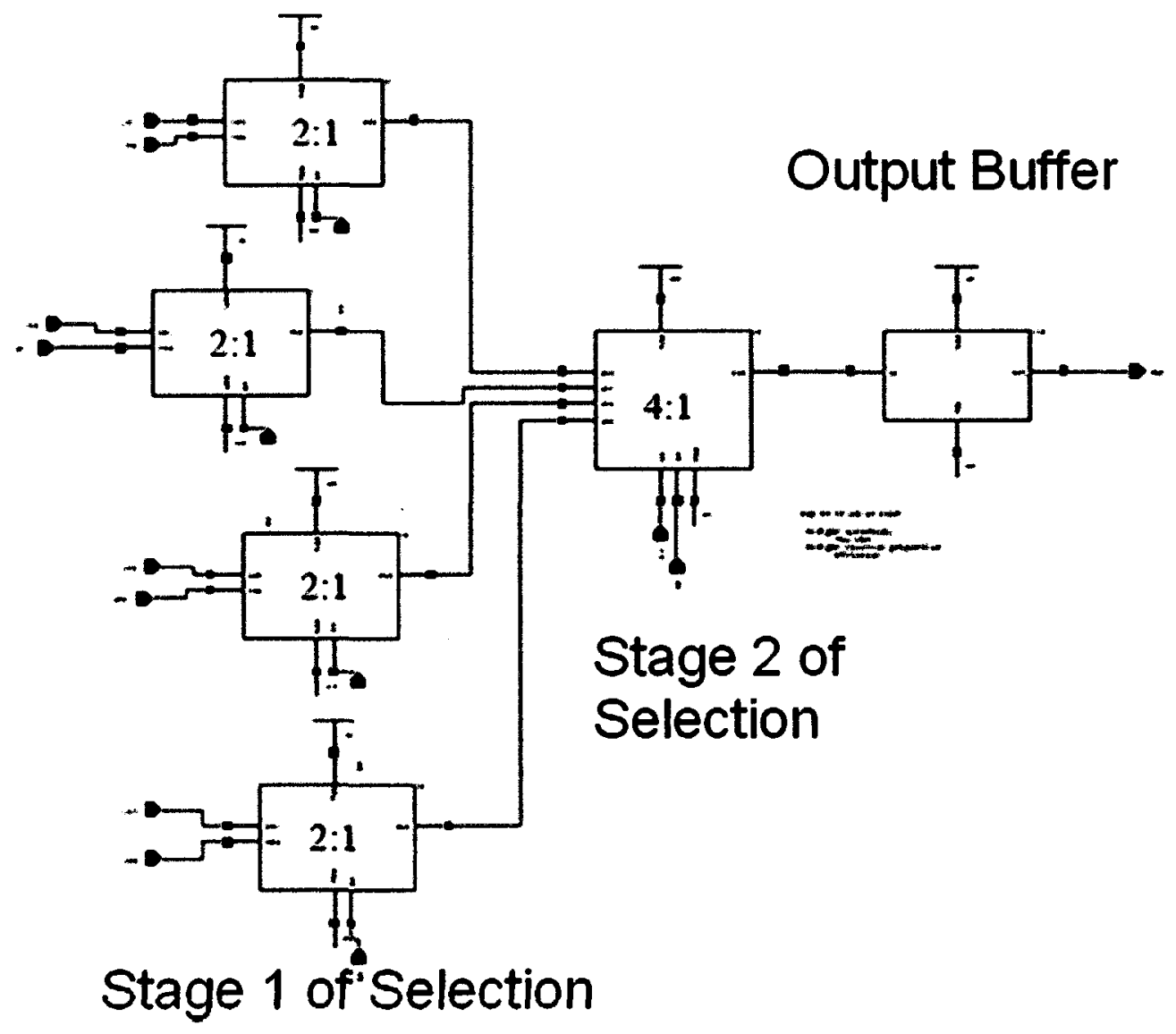

Figure 4-6: Various stages that form the multiplexer chip

The first stage of selection consists of four 2-to-1 multiplexers where one digital bit SO decides whether an even or an odd input is selected. The even or odd inputs are then passed on to a 4-to-1 multiplexer, where two digital bits $\mathrm{S} 1$ and 
Development of EMI Characterization SoP 62

S2 determine which input is passed on to the output buffer and subsequently to the output.

To simplify matters in the layout, the 2-to- 1 multiplexers are replaced by 4-to-1 multiplexers, where two inputs are grounded, and only 2 inputs are connected to the chip pads to receive RF signals. The reason behind this was the availability of a layout block already made for the 4-to-1 multiplexer.

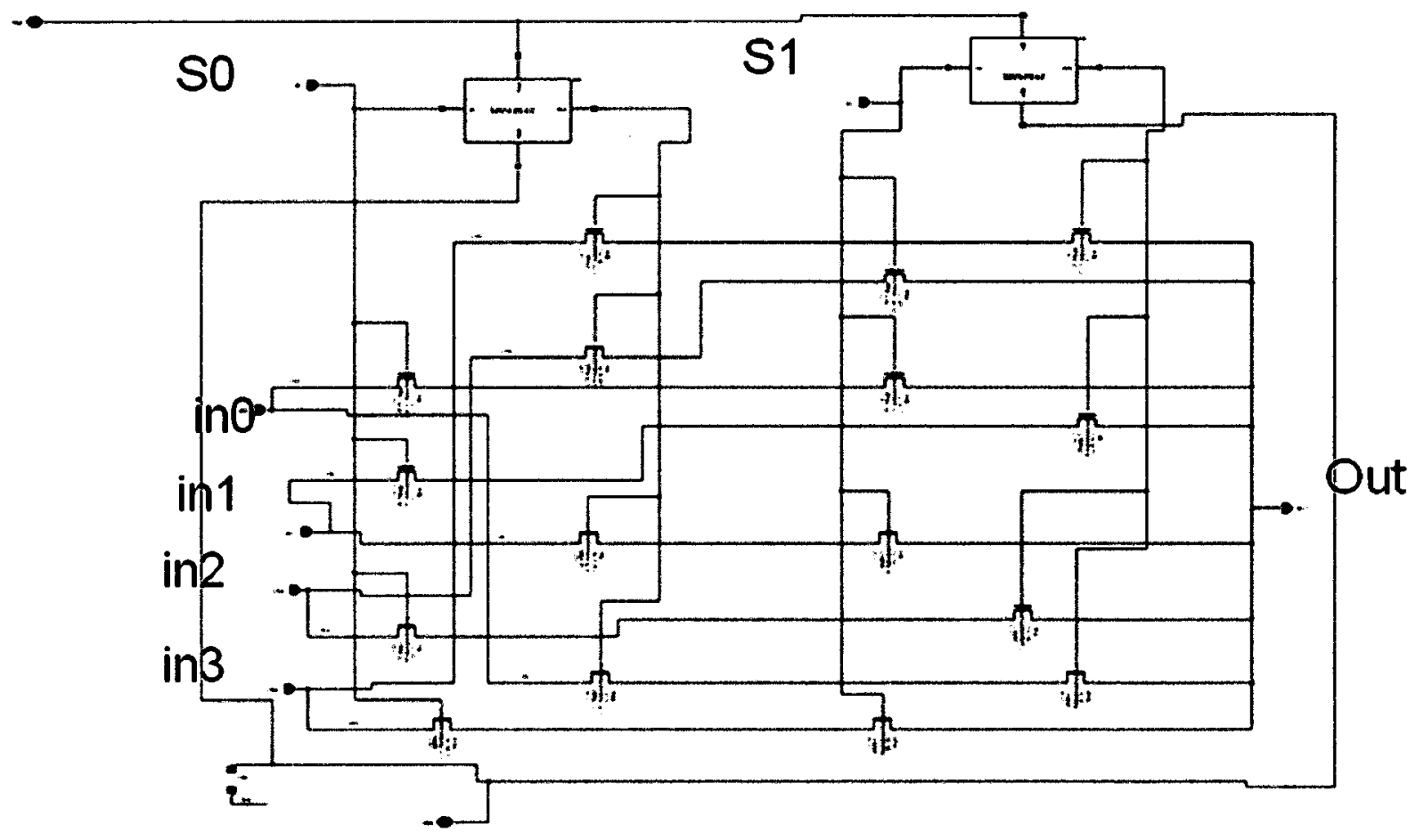

Figure 4-7: 4-to-1 multiplexer circuit

The transistors are made fairly wide in the design of the pass-gates to minimize $R_{d s}$ as given in equation (4-2). The NMOS transistor widths are set to $16 \mu \mathrm{m}$, while the PMOS transistor widths are set to $48 \mu \mathrm{m}$. The lengths for all the transistors were kept to the minimum size of $120 \mathrm{~nm}$ to minimize the parasitic resistances and maximize the cut-off frequency $f_{3 \mathrm{~dB}}$. 
Development of EMI Characterization SoP

To allow for the multiplexer chip output to drive a $50 \mathrm{Ohm}$ load, an output buffer is also designed. The buffer is made of five common source amplifier stages with resistive loading, as shown in Figure 4-8. The resistive loads seen by the drains and sources of the transistors are consistently divided by 2 in the subsequent stage to double the current. In the final stage, the resistance seen by the drain of the last transistor is set to $50 \mathrm{Ohms,}$ which forms with the output termination of 50 Ohms an effective output load of 25 Ohms. An input series capacitor of 8 pF is employed as a DC blocking cap, which allows the passage of the low frequency signal $(100 \mathrm{MHz})$. In addition, a pass gate transistor is added between the blocking capacitor and the output buffer to allow for a clean design rule check (DRC) at the chip level.

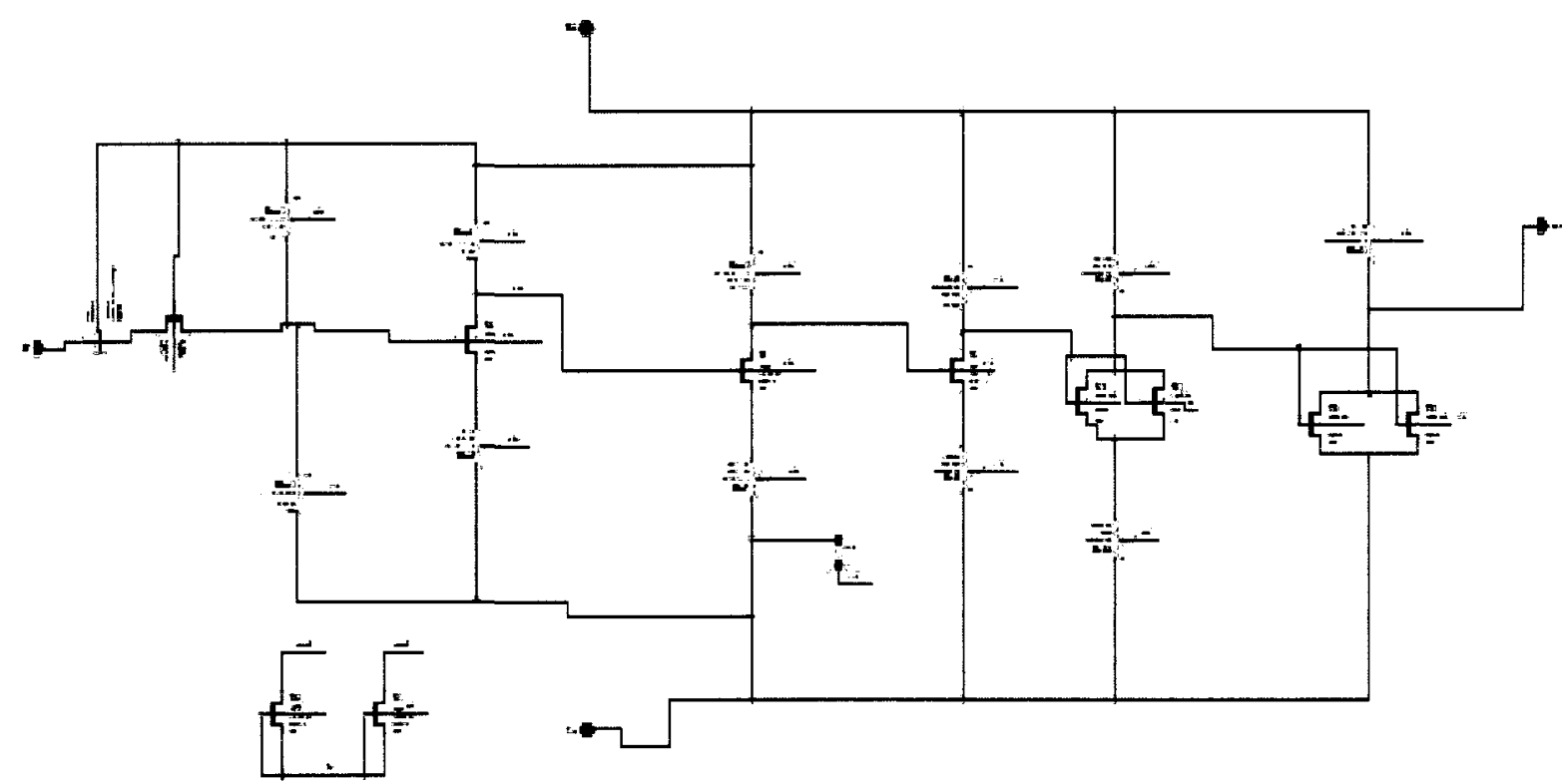

Figure 4-8: Schematic of the output buffer of the multiplexer 


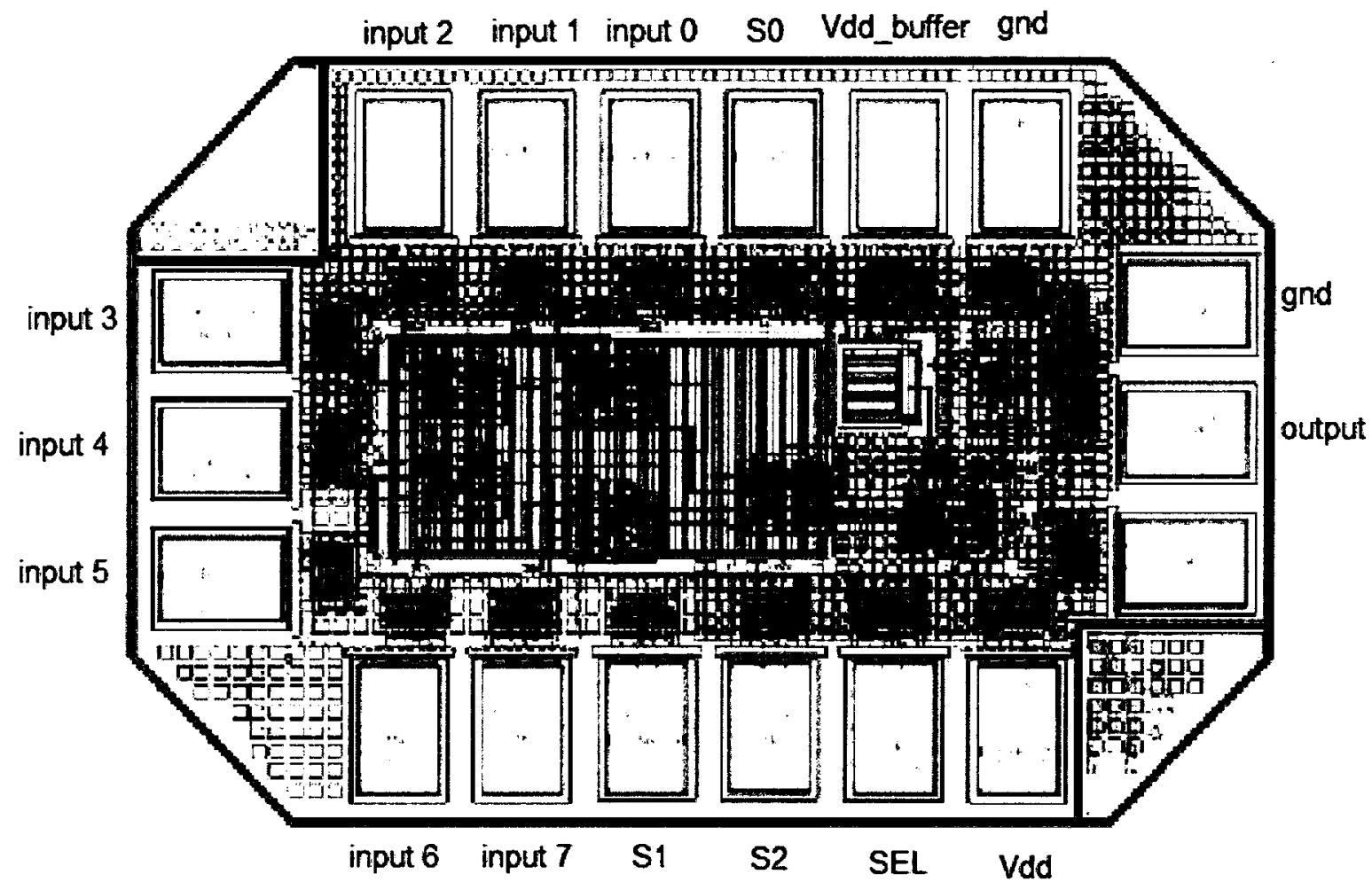

Figure 4-9: Complete layout of the multiplexer chip showing the inputs and the output to the chip.

The layout of the completed chip is seen in Figure 4-9. The pads that are marked as input 0 to input 7 represent the RF input pads to the multiplexer. The pads marked S0, S1, S2, and SEL represent the digital bits that control which RF input is passed to the output. SEL is the chip select input which enables the chip when it is a logic high ( $\mathrm{Vdd}=1.2 \mathrm{~V}$ ) and disables it when it is a logic low ( $\mathrm{gnd}=0 \mathrm{~V}$ ). The supply voltage to the chip is given by Vdd, while the supply for the buffer is given by Vdd_buffer. The gnd is the ground pad which is common for both the multiplexer part and the buffer part. The voltage supply for the pass gates and the buffer were made separate, however the ground was kept the same. 


\section{Simulated performance of the multiplexer chip}

S-parameter simulations were performed to determine the signal loss through each multiplexer chip. $S_{21}$ simulations between input 0 and the output of the chip were done using Spectre RF from Cadence for both the schematic of Figure 4-6 and the extracted layout found on Figure 4-9. The $S_{21}$ plots are shown on Figure 4-10. The negative gain obtained overall (but not the shape of the frequency response) is due to a design flaw that will be explained in section 5.1.2. Further, the simulations show a loss of about $2 \mathrm{~dB}$ between the ideal schematic and the extracted layout. This is due to the parasitic resistances created in the actual signal paths. It is clear that the approximate $1 \mathrm{GHz}$ upper frequency cut-off of the chip is somewhat lower than the $3 \mathrm{GHz}$ desired. This is due to the shunt capacitances associated with the output buffer. However, as was seen in Figure $3-15$, the sensitivity of the cube probes increases significantly with frequency above $1 \mathrm{GHz}$, thereby compensating for the chip's high frequency roll off.

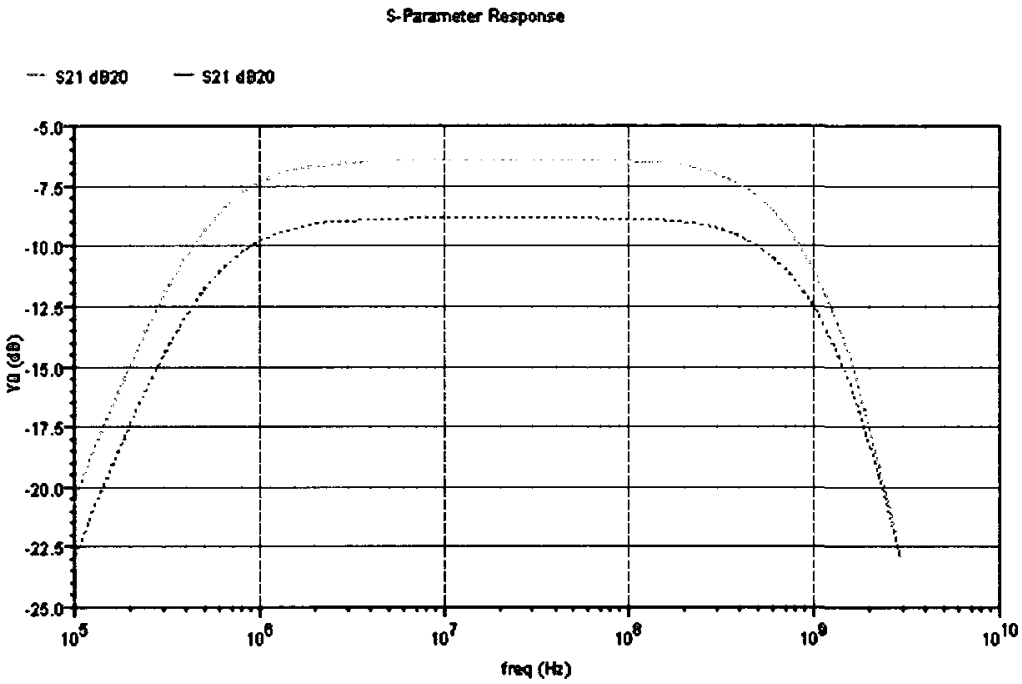

Figure 4-10: S21 plot of the simulated multiplexer schematic and extracted layout. 


\subsubsection{Development of LTCC layout}

A module is designed in LTCC in order to house 64 cube probes and a system of nine multiplexers capable of interrogating each cube probe individually. The goal of the project is to minimize the area required to accomplish the task of integrating the multiplexer chips and the cube probes.

The LTCC module is composed of 19 layers, each having a fired thickness of 95 $\mu \mathrm{m}$. The first layer is empty and is used as a protective layer to prevent the shortcircuiting of the cube probes to the DUT. The cube probes are placed between the layers 2 and 8 . A ground plane is placed on layer 10, two layers above the cube probes. One terminal of each cube probe is connected to the ground plane, and the other is accessed through holes in the ground plane. The ground plane is required to minimize mutual coupling between the probes, as will be further discussed in Chapter 5.

Knowing that the EMI pick up performance of the cube probe does not change with its orientation, a choice is needed to be made in how the adjacent probes are oriented with respect to each other in the array. Also, integrating a chip in a cavity placed above the cube probes, while respecting the LTCC technology rules, forces the adjacent probes to have an orientation offset by 180 degrees. Simulations were performed in Ansoft HFSS in order to determine the difference in mutual coupling that occurs between the adjacent elements as a function of their orientation. Two orientations are considered: 0 or 180 as shown in Figure 4-11. Figure 4-12 shows the mutual coupling results as found by HFSS for both orientations. By inspection of the figure, it is evident that when the cube probes 
Development of EMI Characterization SoP

have the same orientation $\left(0^{\circ}\right)$, the mutual coupling $S_{21}$ (measured between port

1 connected to one probe, and port 2 connected to the second probe) is lower by almost $5 \mathrm{~dB}$ at frequencies below $3 \mathrm{GHz}$. Also, when the operating frequency exceeds $3 \mathrm{GHz}$, the mutual coupling between the cube probes in opposite orientations is lower. Since the SoP is to be operational between $0.1 \mathrm{GHz}$ and 3 $\mathrm{GHz}$, it is preferable to keep the same orientation for all the elements in the array. However, it is more important to have the adjacent cube probes oriented differently $\left(180^{\circ}\right)$ so as to ensure the positioning of a cavity directly above the elements. This also represents a trade-off between by minimizing the RF path losses and mutual coupling between the mutual coupling between the adjacent elements, and the module's size.

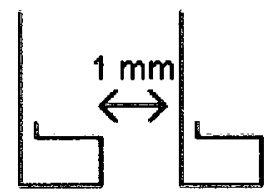

a)

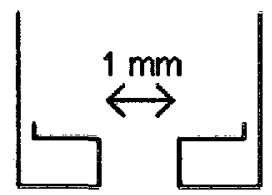

b)

Figure 4-11: a) Cube Probes with an orientation difference of 0 , b) Cube Probes with an orientation difference of 180 


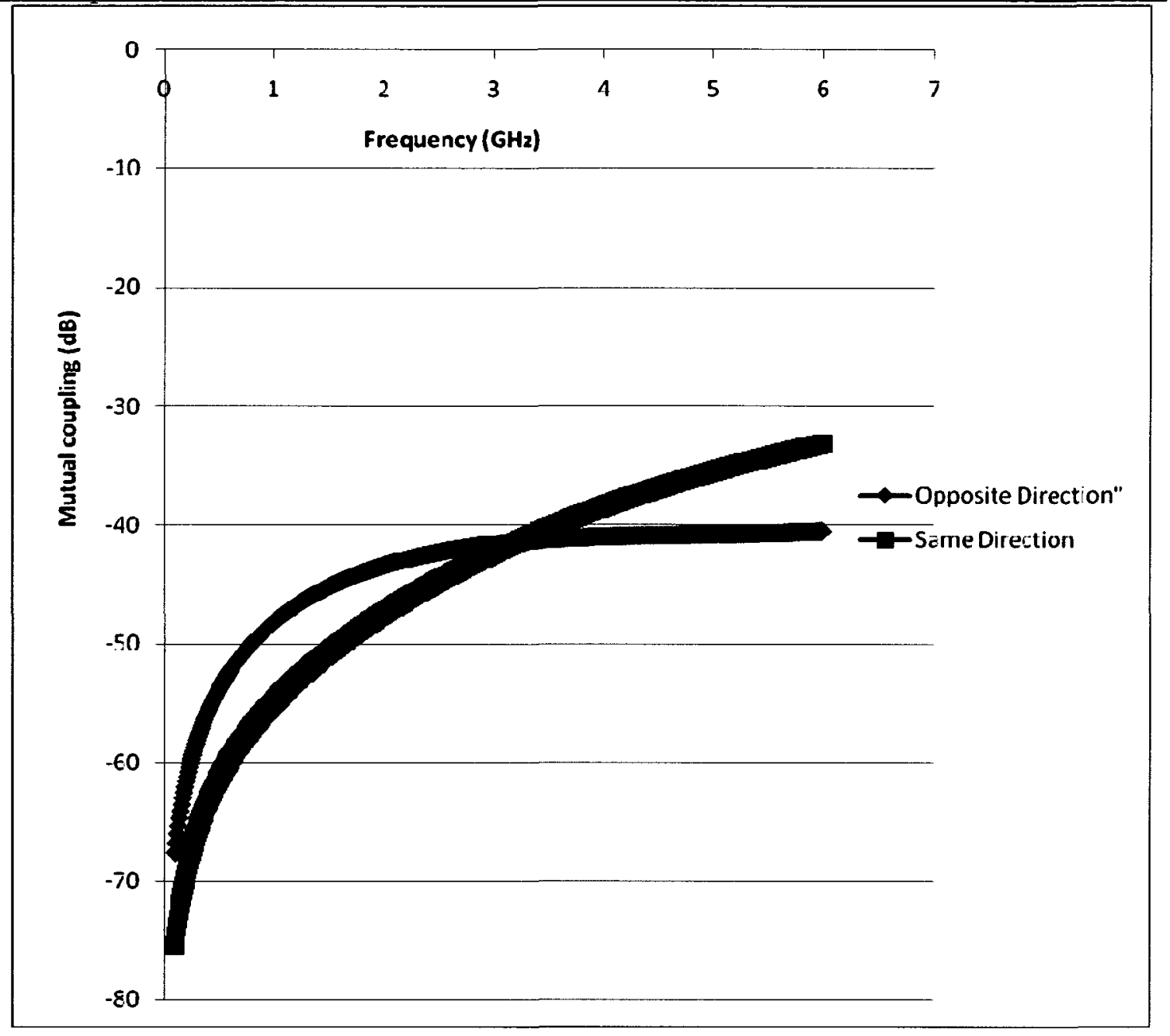

Figure 4-12: Mutual coupling simulation results showing the mutual coupling in dB for frequencies between 0.1 and $6 \mathrm{GHz}$ for probes in opposite orientation (blue) and same orientation (red).

Figure 4-13 shows the cube probes (in blue) once placed in the SoP as seen from the top. The cube probes are placed in the array such that the relative orientation between the adjacent elements is $180^{\circ}$. 


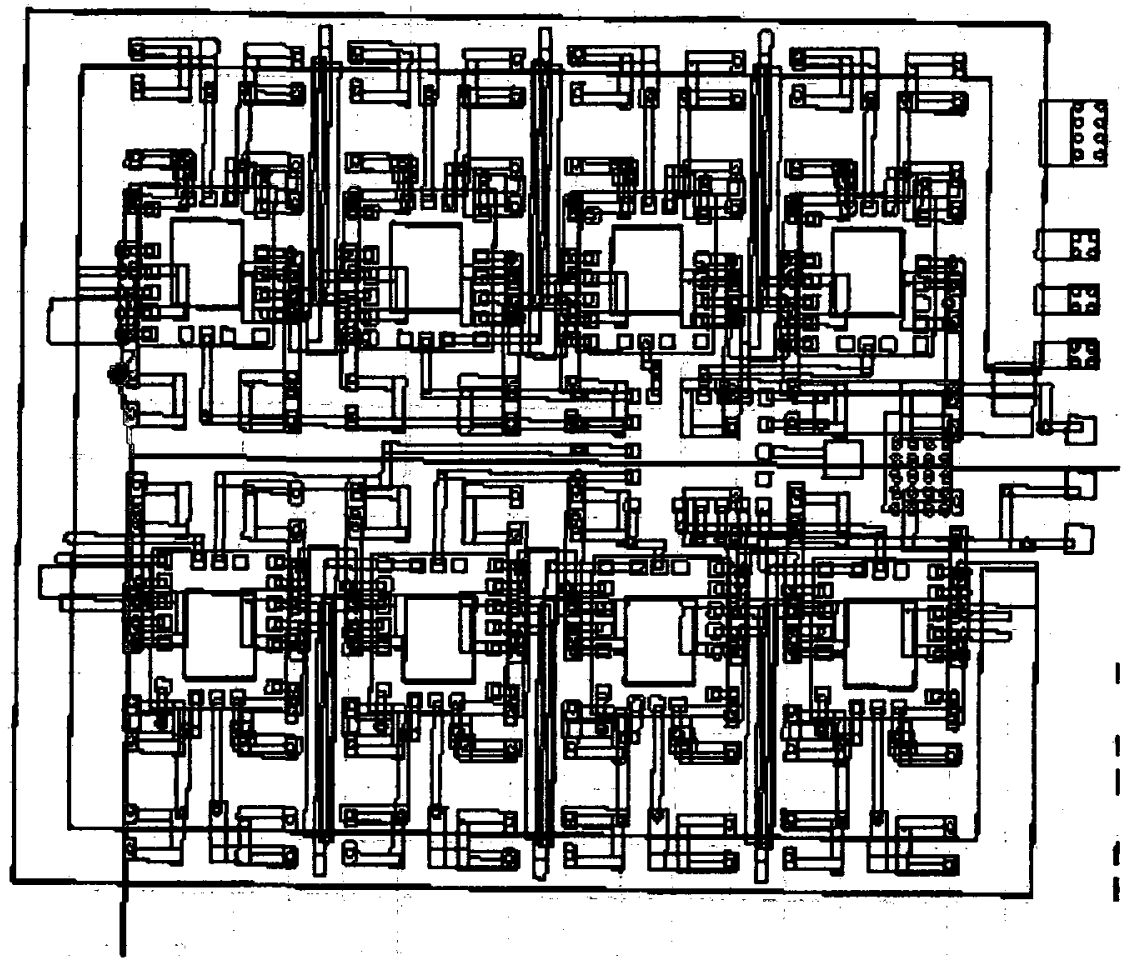

Figure 4-13: Cube probes placed in the LTCC layout.

The connections between the cube probes and the multiplexers, placed in the cavities, take place between layers 11 and 17. On the layers 18 and 19, the multiplexers in the cavities are connected to the ninth multiplexer which then passes the required RF signal to the output. The pads for connecting to the control line inputs are present on the surface, or above layer 19, as are the pads for the ground and the supply voltage Vdd. Figure 4-14 shows a complete diagram of the LTCC module idnetifying all the control pads, the RF output and ground pads, the ground pad, and the supply voltage Vdd pad. 


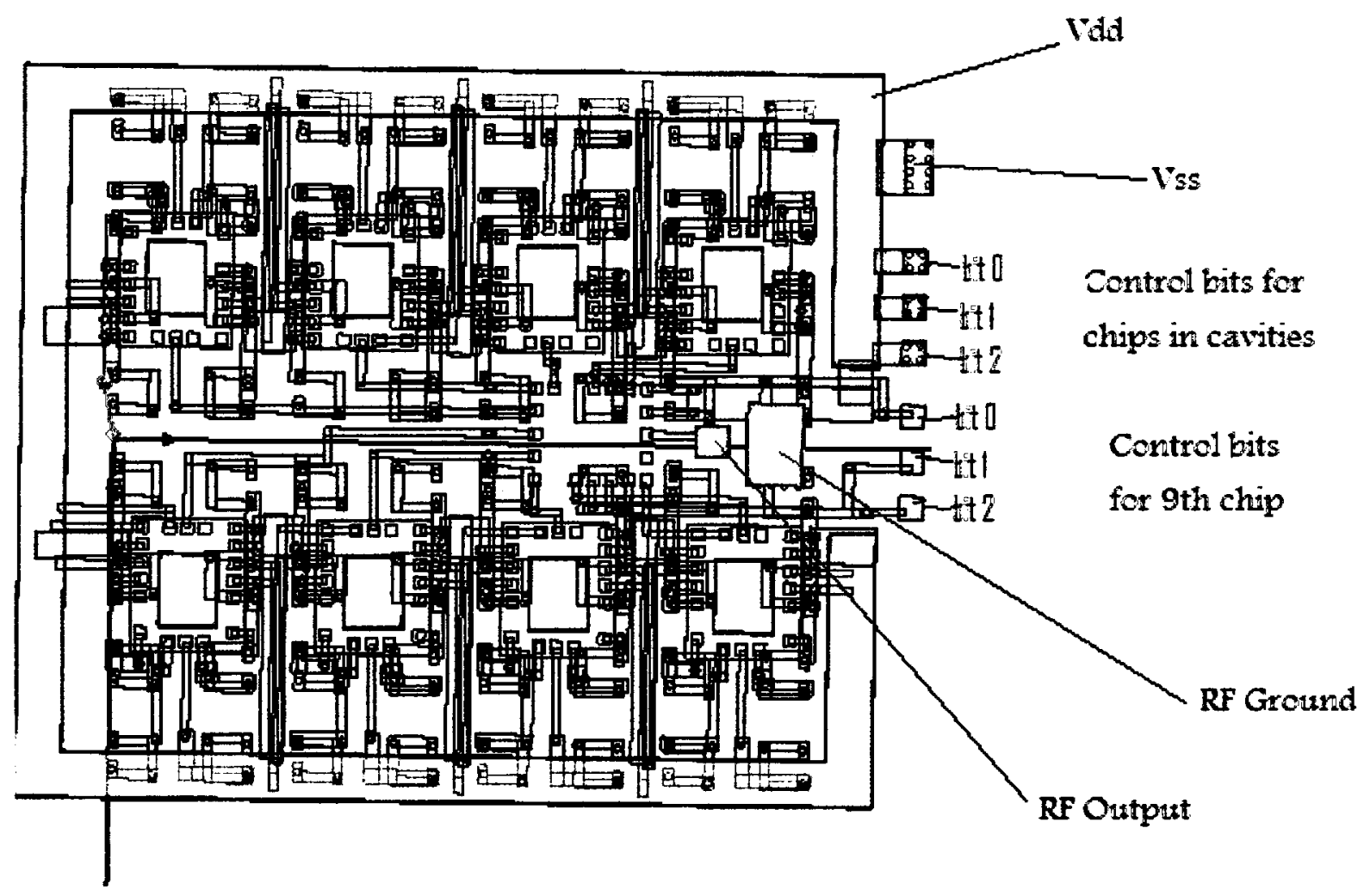

Figure 4-14: Complete SoP layout showing the pads for the various external connections

Eight of the nine multiplexers are placed in cavities while the $9^{\text {th }}$ multiplexer that connects to the output is placed as a surface mounted chip. The reason for this is the lack of space to create a cavity in the middle of the SoP. Available space is an issue given that the bottom layers are defined by the $1 \mathrm{~mm}$ spacing between the cube probes and their sizes. Therefore, the array of cube probes determines the size of the module. Figure 4-15 shows the fabricated LTCC module with the populated multiplexer chips. 
Figure 4-15: Fabricated LTCC module populated with multiplexer chips 


\section{Chapter 5}

\section{Assessment/Experimental Validation of LTCC SoP}

To assess the EMI/EMC characterization tool SoP, complete experimental validation of the module is necessary. This chapter begins with the evaluation of the multiplexer chip, followed by the validation of the performance of cube probes in an array.

The testing of the multiplexer chips and the total system will be presented. To this end, the SoP will be placed above a microstrip transmission line and the parasitic EMI energy radiated by this source will be measured.

\subsection{Testing of the Multiplexer Chip}

The testing of the multiplexer chip requires the ability to perform $S_{21}$ measurements between an input port and the output port of the chip to determine the insertion losses that are incurred as a function of frequency. With the space allocated from CMC for the chip realization, placing appropriate test pads inside the chip for "on-wafer" measurements was impossible. The characterization of the multiplexer chip must also include the quantifying of isolation between the neighbouring input ports to the multiplexer. Therefore, the only way to test the multiplexer chip is through the use of an RF test fixture. Conveniently, CMC microelectronics offers an SMA connectorized test PCB jig that is adapted to a CQFP80 pin package. 
Therefore, five sample multiplexer chips were packaged in 80-pin CQFP-80-VA packages. A packaged chip clamped to the CFP80TF test fixture is shown in Figure 5-1.

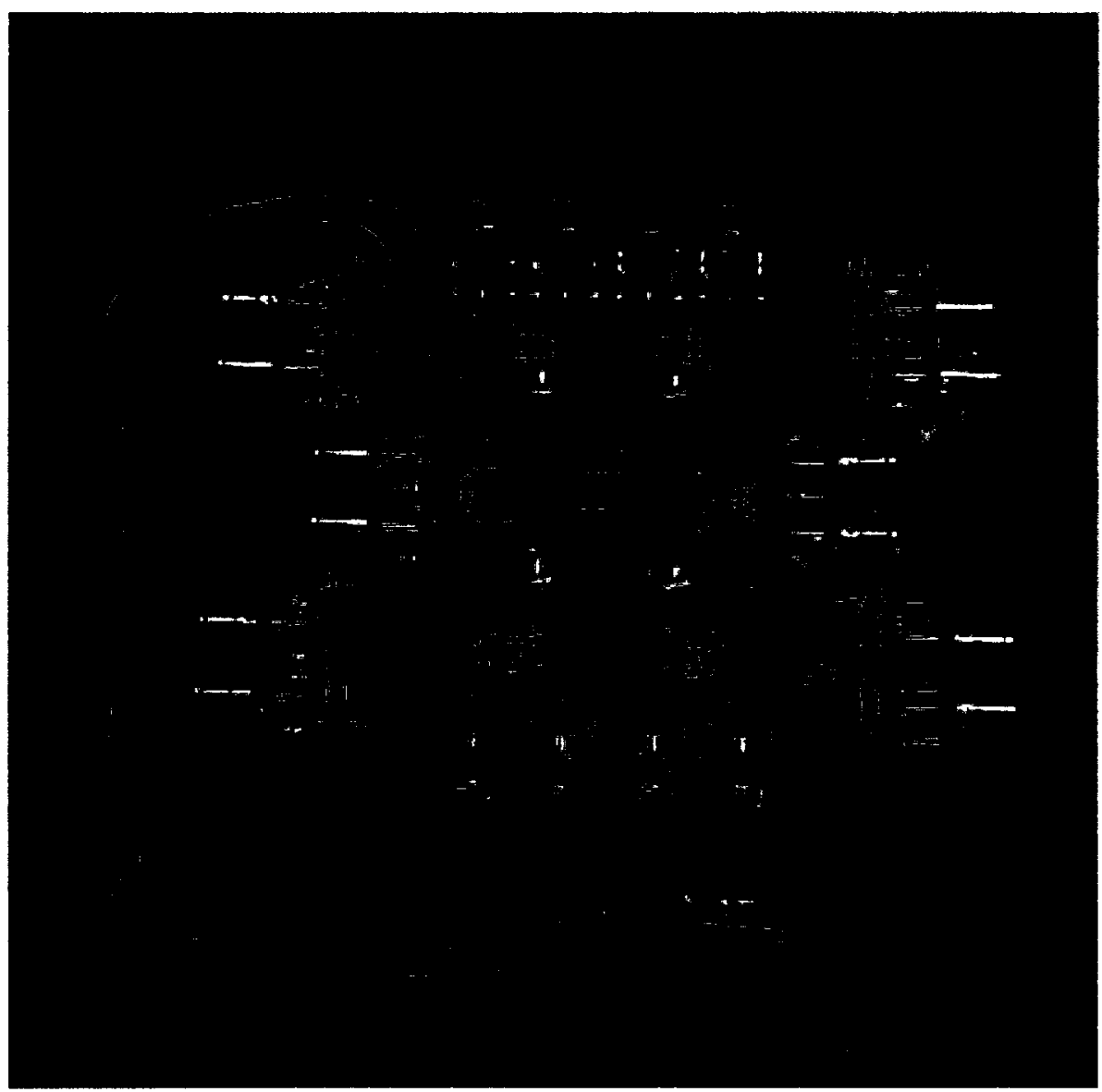

Figure 5-1: Packaged chip clamped to a CFP80TF test fixture

The wire bonding of the chip to the CQFP-80-VA package is done through CMC.

Figure 5-2 shows the wire bonding plan employed. Four RF inputs were wirebonded, in addition to the three control bits (SO, S1, S2), the voltage supplies (Vdd), both ground connections (GND), and the RF output. 


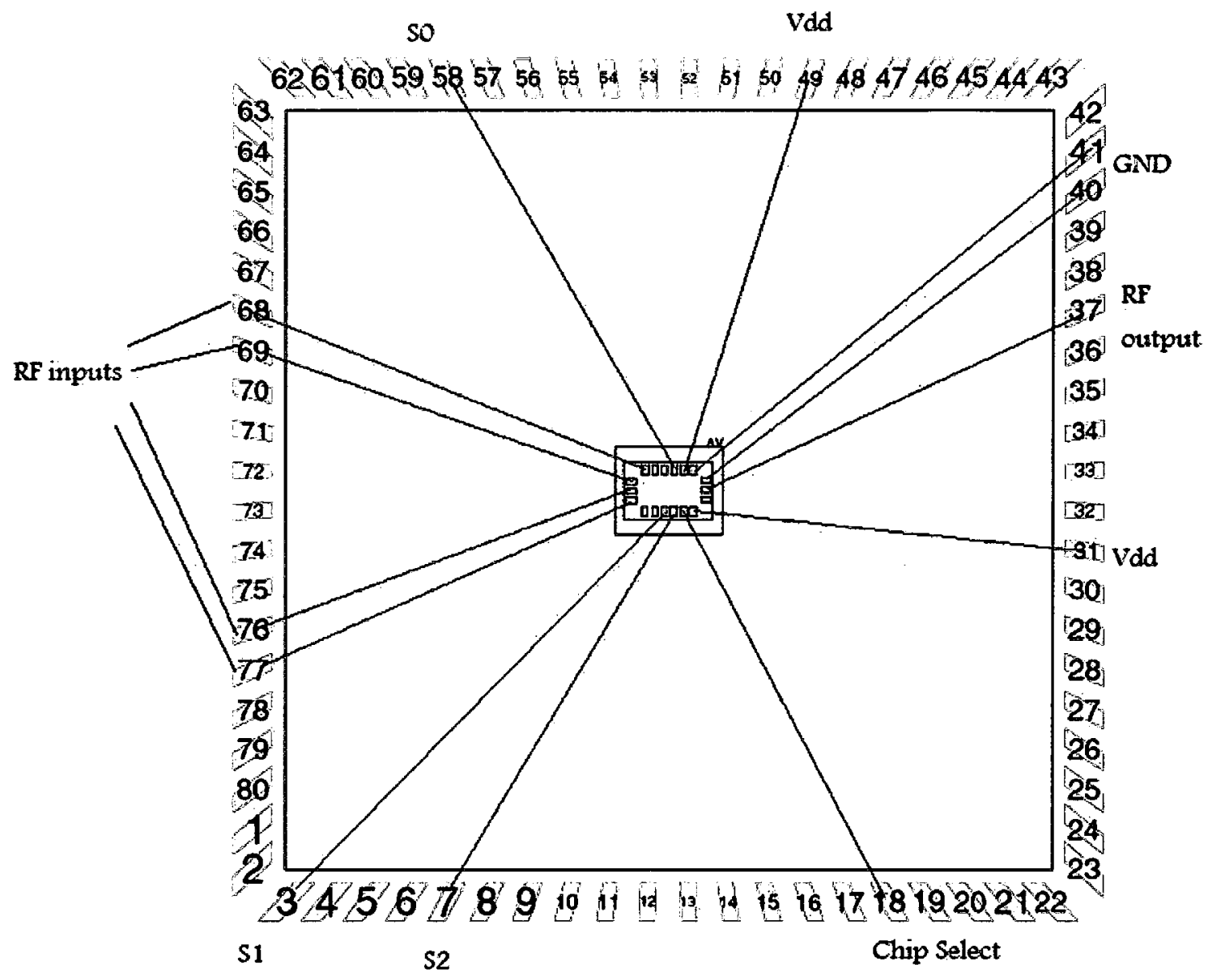

Figure 5-2: Wire bonding plan submitted to CMC identifying the wire-bonded pins.

\section{Multiplexer Chip test results}

A measurement of $S_{11}$ at an input port was performed to determine the return loss in a $50 \mathrm{Ohm}$ system. In addition, multiple measurements of $\mathrm{S}_{21}$ were performed between various inputs to the multiplexer and the output port. Figures 5-3 and 5-4 show the return loss $S_{11}$ and the insertion loss $S_{21}$, respectively, measured using a vector network analyzer. It should be noted that the current drawn by the chip was flickering between the desired $25 \mathrm{~mA}$ and lower values during the measurements (as will be explained later) with a supplied voltage of 
$1.4 \mathrm{~V}$ instead of the $1.2 \mathrm{~V}$ design value. The additional voltage compensated for the losses through the wires and lines connecting to or on the printed circuit board.

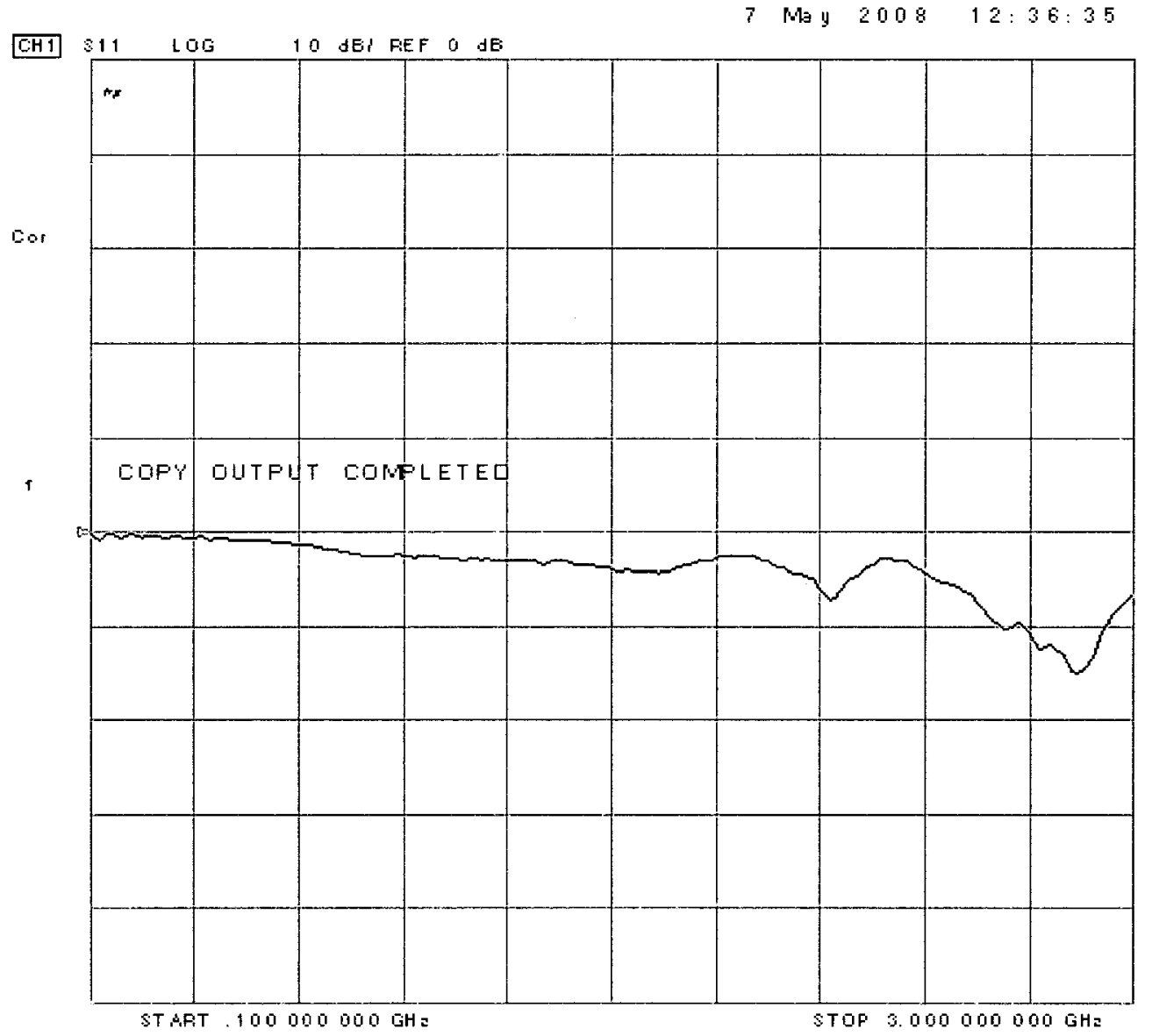

Figure 5-3: Measured $S_{11}$ of the multiplexer input port through the test fixture.

The $S_{11}$ results obtained show a very high return loss at low frequencies, diminishing above approximately $1 \mathrm{GHz}$. This low frequency behaviour is to be expected based on the equivalent transmission gate model shown on Figure 4-3 (b) and for its input impedance. At higher frequencies, the board and package parasitic effects are substantial and the model shown in Figure 4-3(b) is no longer sufficient. 


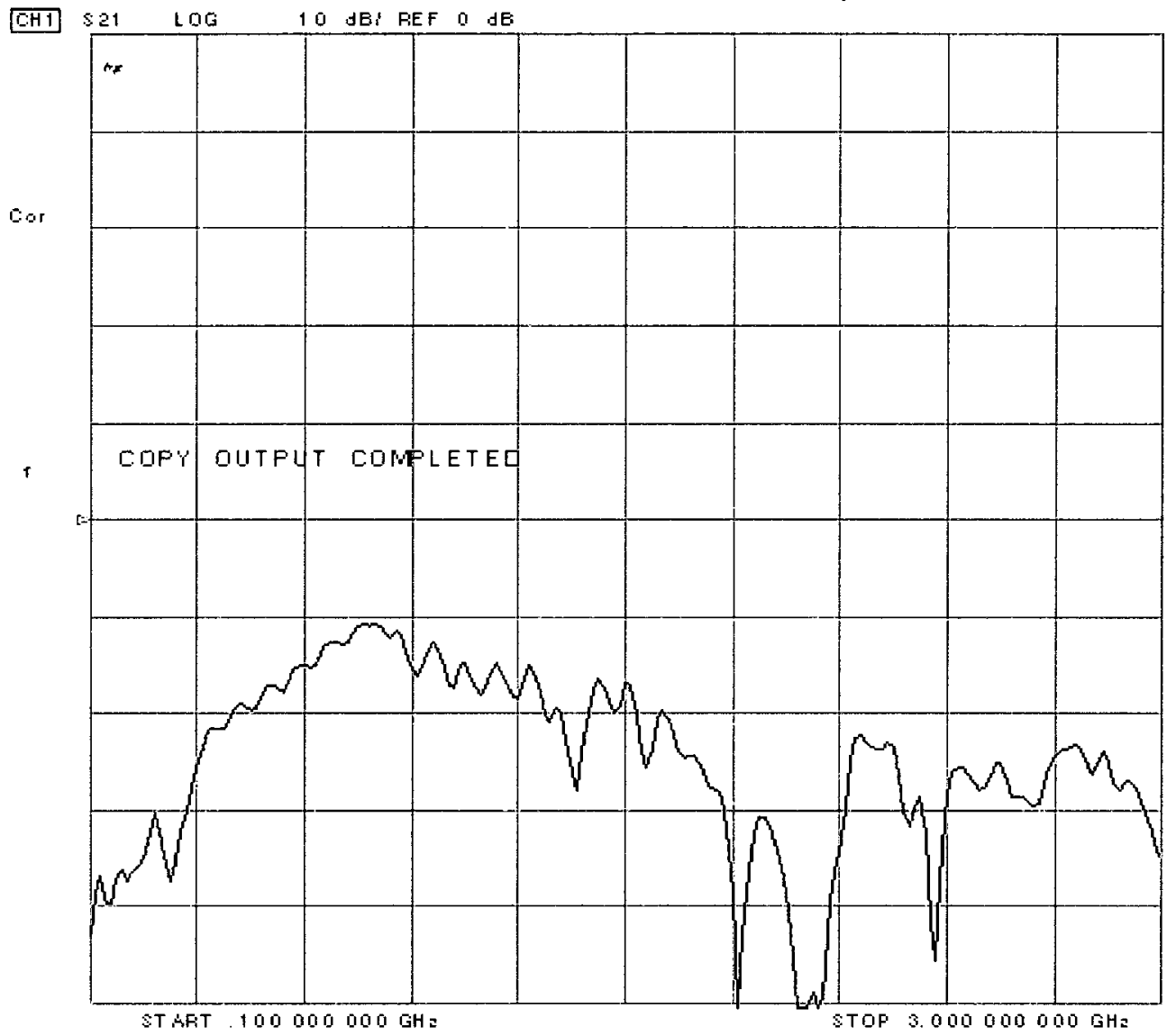

Figure 5-4: Measured $S_{21}$ of the multiplexer chip through the test fixture

In terms of Insertion loss performance obtained in Figure 5-4, a number of comments must be made. First, a relatively close agreement with the simulated results of Figure 4-10 is obtained above $800 \mathrm{MHz}$, i.e. a maximum passband gain of about $-10 \mathrm{~dB}$ and an upper cut-off frequency of $1 \mathrm{GHz}$. Second, as was the case with the $S_{11}$ measurement, significant parasitic effects are present at higher frequencies. Third, there is a considerable offset between the measurements and simulation below $800 \mathrm{MHz}$. The drop-off in the measured response at low frequencies can be attributed to the presence of a small series capacitance due 
Assessment/Experimental Validation of LTCC SoP

to the contact-less nature of the CQFP-80-VA package mounting to the test fixture.

Indeed, any small misalignment can introduce an air gap that would produce a series-capacitor like effect. Given the limited availability of the test fixture and the restrictions on its use (preventing the soldering of the package leads that would remedy the problem), it was decided to not pursue the multiplexer chip testing any further.

It is clear that the observed performance of the chip will vary depending on its environment, and it is the multiplexer's operation within the LTCC SoP that is of greater interest. In addition, a de-embedding process is needed to find the actual return and insertion losses of the chip alone, since the effect of the package and the test fixture is not completely accounted for in the previous measurements. To perform the de-embedding measurements, identical packages with calibration structures are required which are not available.

Finally, the isolation between two input ports of the multiplexer chip is determined. This is done by selecting one transmission path while blocking the other and measuring the difference in transmission losses of the two paths. At 1 $\mathrm{GHz}$, the isolation was found to be neighbouring - $10 \mathrm{~dB}$. Due to time constraints, the full characterization of isolation over frequency was not done.

\section{Analysis of the multiplexer chip performance}

While the S-parameter results of the chip are discouraging, it was found that the cause of the low insertion gain is a design flaw. Originally, the chip was 
Assessment/Experimental Validation of LTCC SoP

supposed to have a large gain to boost the low voltage signals received by the cube probes. Therefore, further losses render the detected EMI very hard to process due to the burial of the signal in the noise.

During the tape out of the multiplexer chip, and on the last day of submission, a design rule check (DRC) error was not allowing the connection of the buffer's input to its DC voltage supply pad. The error is called an antenna rule. The reasoning behind the error is that during the processing of the wafer, a potential large amount of current can run through the pads, causing a transistor breakdown if the current flows through the transistor's gate. The typical design procedure, to avoid this DRC error from occurring, is to connect a small metal line from the voltage pad in question to the ground. This way during the processing, the current will simply flow to the ground. When the circuit is to become operational, the metal line can then be removed using lasers.

However, due to the designer's lack of experience, a different solution was found to overcome the antenna rule DRC problem. The error was removed with a transistor connected as a pass-gate between the DC coupling capacitor and the input of the first stage of the buffer. The gate of the transistor was connected to the voltage pad labeled Vdd_buffer which was causing the DRC error.

The intended operation is that the pass-gate only acts as a resistor, therefore solving the problem and not changing other aspects of the circuit. The chip was therefore submitted without simulating this minor circuit modification. The DRC error was successfully removed, but the end result was a buffer exhibiting loss as opposed to gain. It was later realized that the problem with the NMOS pass-gate 
Assessment/Experimental Validation of LTCC SoP

is when the voltage at the source is greater than the voltage at the drain, it acts

like an open switch. Therefore, when the first stage attempts to amplify the signal, it shuts off the transmission through the pass-gate, rendering the operation lossy. This reasoning explains the flickering of current consumption as observed during the S-parameter measurements taken while using the test fixture. Figure 5-5 shows the difference in the insertion gain of the buffer when it is operating in its desired fashion compared to when the pass-gate is utilized. Note that the overall multiplexer performance as submitted is given in Figure 410.

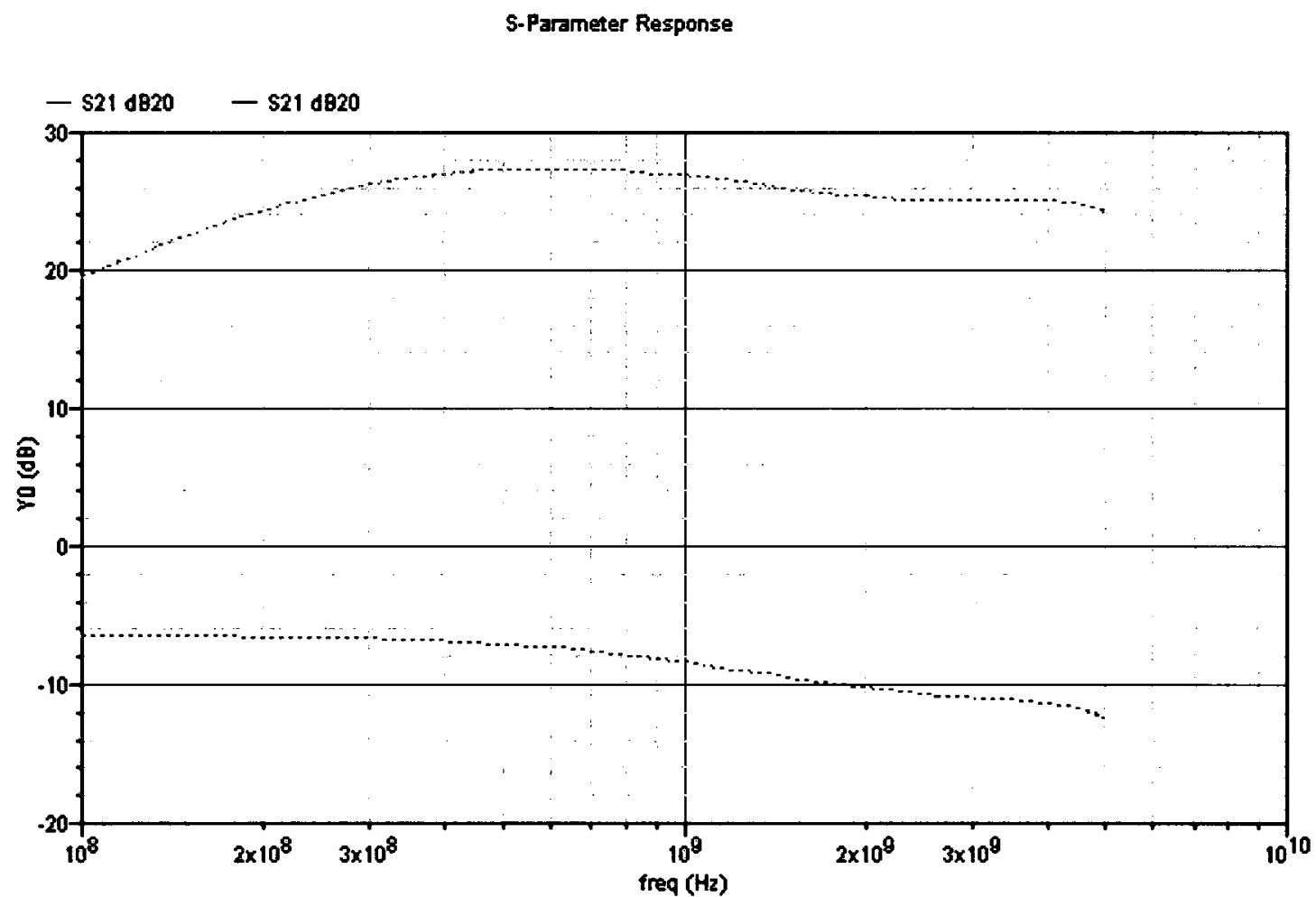

Figure 5-5: Comparison of the insertion gain of the multiplexer chip's buffer with and without DRC required modification 
The results shown in Figure 5-5 are representative of the insertion gain when the buffer is operating normally (higher curve) and when the buffer is operating with the DRC fix (lower curve). The difference is on average around $25 \mathrm{~dB}$, and in some cases greater than $30 \mathrm{~dB}$ depending on the frequency of operation. This result shows that the chip would need very minor tweaking in a re-design to work well for the EMI SoP application.

\subsection{Array Characterization}

The performance of the cube probe has been documented in Chapter 3 of this thesis. While the cube probe alone was fit for EMI measurements, it remains to be seen whether its behaviour varies in an array of similar elements. For the purpose of the development of an EMI sensor, an inter-element spacing of $1 \mathrm{~mm}$ was chosen for the array of cube probes. The ideal behaviour is when each cube probe is not affected by its neighbours. It is important to determine any change in $\mathrm{H}$-field pickup between a stand-alone probe and the same probe in an array. Furthermore, it is important to test the input impedance of a cube probe when surrounded by other probes in the LTCC array, such that future co-design between the multiplexer chip and the probes may take this in consideration. Most of the characterization performed in this section is done using Ansoft HFSS which was validated in Chapter 3 for the cube probe structures. 


\subsubsection{H-field EMI pickup in an array}

The array of cube probes is meant to replace the EMI scanning operation using a scan table. Each element in the array should pickup the same amount of $\mathrm{H}$-field as a stand-alone element over a device under test. Thus the replication of a magnetic field probe displaced over a DUT would be achieved.

Using Ansoft HFSS, a simulation was performed to determine the difference in the pickup of a cube probe when placed alone or in the presence of other elements in close proximity. The simulation was performed with the cube probe situated $1 \mathrm{~mm}$ above $(z=1 \mathrm{~mm})$ and $1 \mathrm{~mm}$ to the side $(x=1 \mathrm{~mm})$ of a reference microstrip line DUT. The same simulation setup as in Figure 3-6 is employed. Figures 5-6 and 5-7 show the simulation results for both cases.

Figure 5-6 shows the result of the first simulation where an isolated probe is suspended $1 \mathrm{~mm}$ above (on the z-axis) a 50 Ohm terminated microstrip line and $1 \mathrm{~mm}$ to the side (x-axis) of it. Similarly to Figures 3-7 and 3-15, the probe's pickup increases with frequency.

The same simulation was then repeated for a cube probe located in the centre of an array of identical probe elements. The lower curve (red) of Figure 5-7 shows this result. It can be seen that the difference in pickup levels between the two cases is less than $0.3 \mathrm{~dB}$, which is negligible for EMI diagnostics. Therefore, it can be concluded that the energy pickup levels of a cube probe does not vary significantly in a simulated array.

The upper curve (purple) of Figure 5-7 shows the pickup of the closest neighbouring probe situated directly above the microstrip line, i.e. at $(z=1 \mathrm{~mm}, x$ 
Assessment/Experimental Validation of LTCC SoP

$=0 \mathrm{~mm}$ ). Here a difference of approximately $3 \mathrm{~dB}$ is observed between two

adjacent elements, confirming that the array is indeed picking up varying $\mathrm{H}$-field

levels as a function of the spatial intensity of the source.

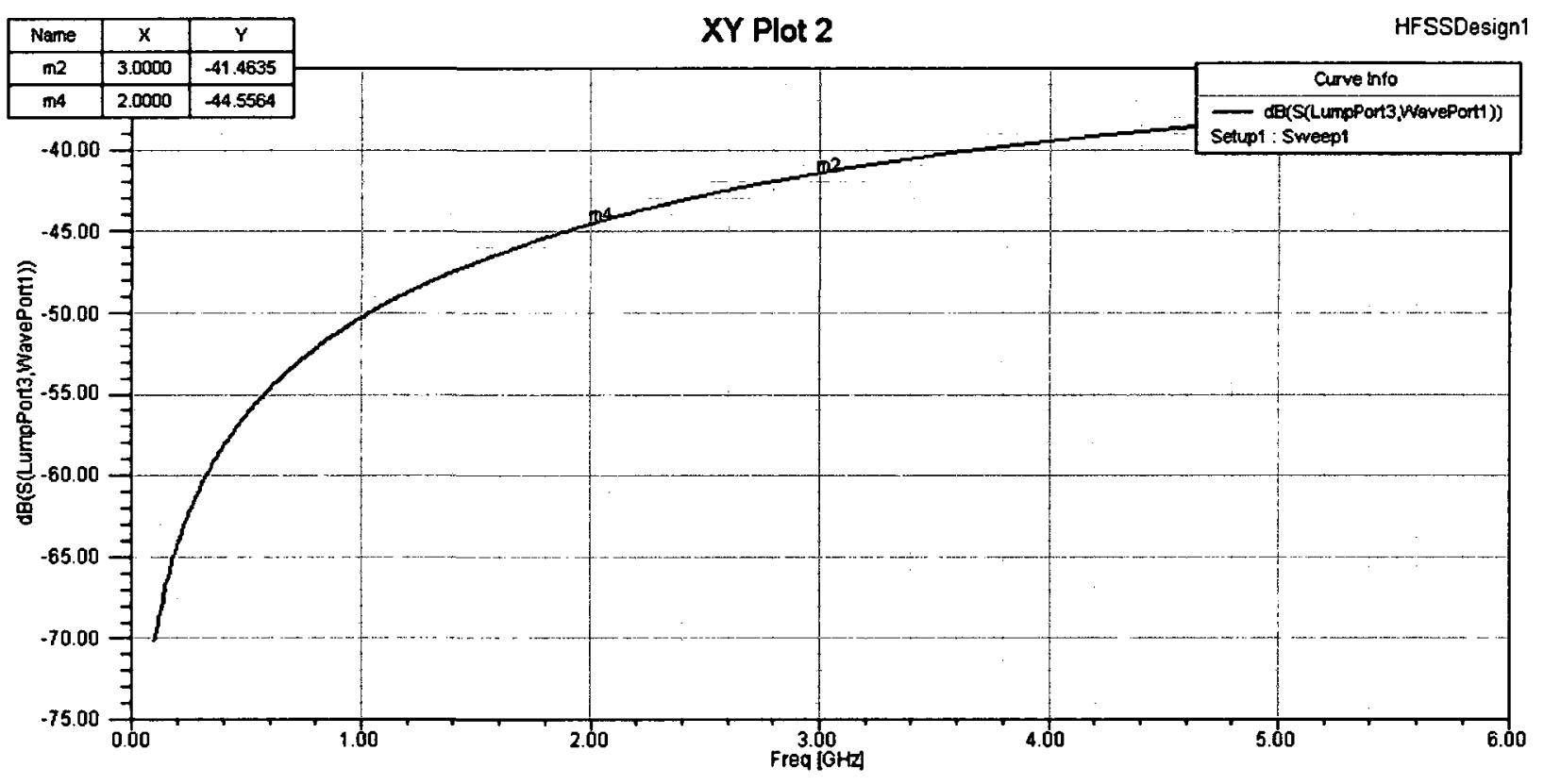

Figure 5-6: Pick up of an isolated cube probe (probe placed at $\mathrm{z}=1 \mathrm{~mm}, \mathrm{x}=1 \mathrm{~mm}$ )

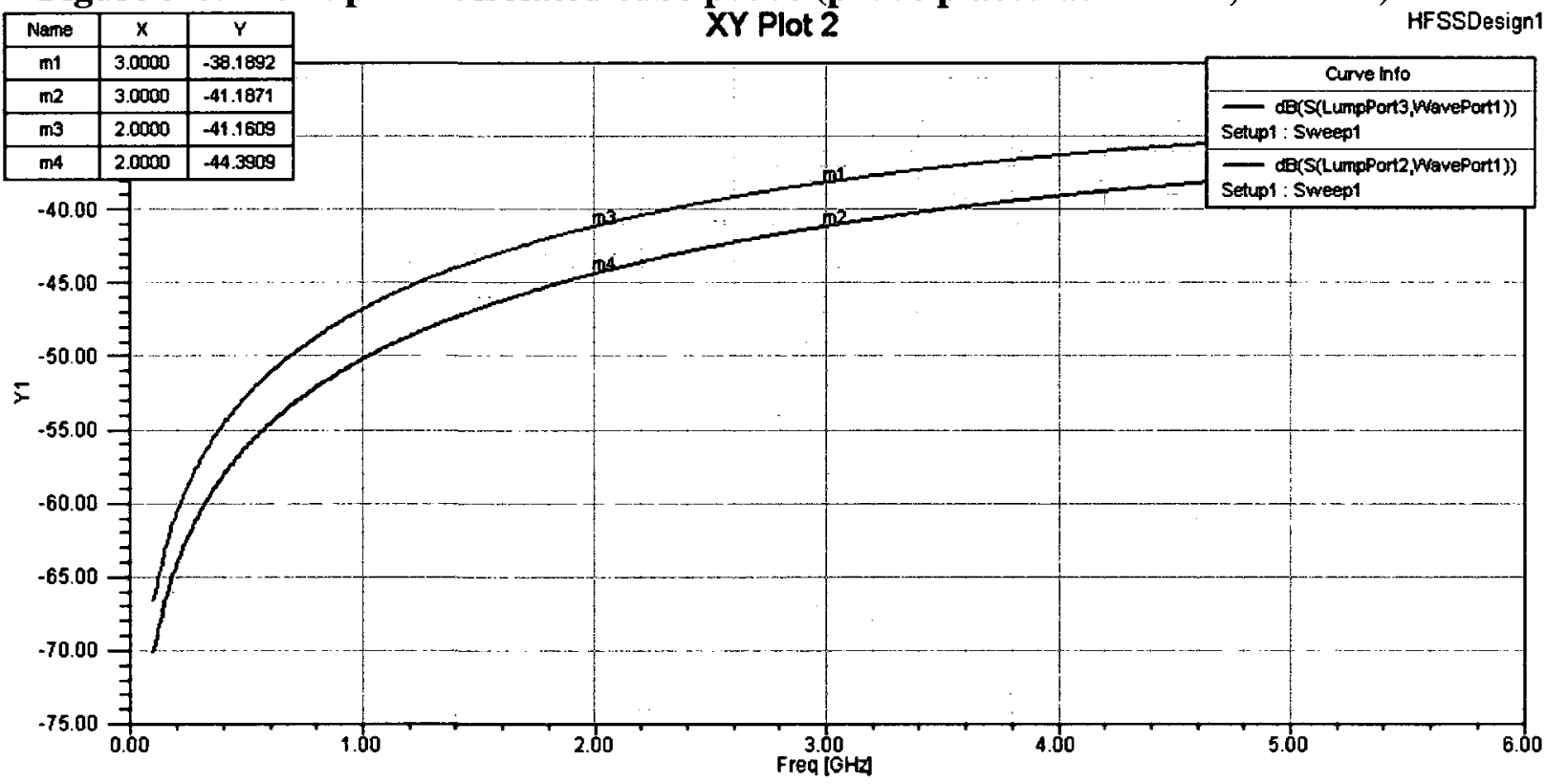

Figure 5-7: Cube probe pickup in a 9 element array for probe placed at $z=1 \mathrm{~mm}$, $x=1 \mathrm{~mm}$ (red) and $\mathrm{z}=1 \mathrm{~mm}, \mathrm{x}=\mathbf{0 m m}$ (purple) 


\subsubsection{Input Impedance in an array}

The cube probe's input impedance in an array of 9 elements was verified for three different conditions of terminating impedances of its neighbours:

- All neighbouring cube probes are shorted to ground

- All neighbouring cube probes are open circuited on one end and connected to a common ground plane on the other.

- All neighbouring Cube Probes are connected to a 50 Ohm load on one end and to a common ground plane on the other.

The purpose of the three configurations is to present three different environments on the Smith Chart. The first condition is to ensure a high current flow in the shorted cube probes. The second variation with the open circuited cube probes is to eliminate current flow from the neighbouring probes. The third configuration, using $50 \mathrm{Ohm}$ loads as terminations, presents an intermediate operation between the first two. This allows for a smaller current flow in the neighbouring probes in comparison with the case when the probes are shorted.

The array characterization was performed by means of both simulations and measurements. To determine whether the simulations provide accurate results, two test structures were built in LTCC for experimental verification. The first test structure consists of a cube probe in the middle of eight other probes that are all shorted together. The second test structure consists of a cube probe in the middle of eight other probes that are open circuited. The third structure involved finding $50 \mathrm{Ohm}$ loads that are suitable for surface mounting and small enough to 
Assessment/Experimental Validation of LTCC SoP

connect to the cube probe. This became complicated to realize and was therefore abandoned.

Figure 5-8 shows the layout of the two test structures as submitted to for fabrication. The upper part of Figure 5-8 shows all the peripheral cube probes shorted together on the top layer. In addition, some space is left between both tips of the Cube Probe that is to be measured. On one end, the cube probe under test is connected to a gold conductor, and the opposite end is connected to another gold conductor that employs a via fence to secure a solid connection to the ground layer. A surface mount RF connector is utilized to measure the input impedance of the cube probe by having the signal pin connected to the conductor metal and the ground pin connected to the grounded conductor.

The lower part of Figure 5-8 shows the test structure employing the open circuited cube probes. The only difference between the second test structure (lower part of Figure 5-8) and the first test structure (upper part of Figure 5-8) is in the absence of a surface metal shorting the peripheral cube probes. Thus, all the peripheral cube probes have one end open circuited, and the other end connected to a ground plane. For the purpose of measuring the input impedance of the middle cube probe, one of its ends was connected to a metal conductor, and the opposite end connected to a grounded conductor. 

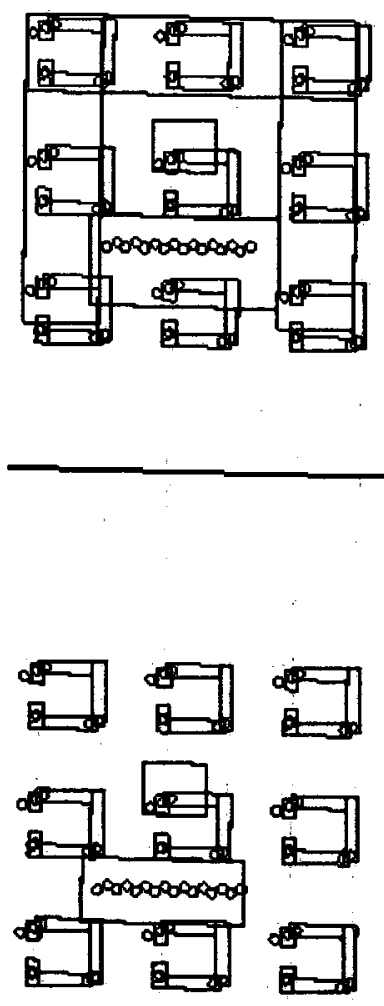

Figure 5-8: Two array test structures built in LTCC with short circuited Cube Probes (upper part) and open circuited Cube Probes (lower part)

\section{Cube Probe in an array of short circuited Cube Probes}

The first simulated array configuration is of the cube probe in the middle of eight peripheral cube probes all shorted together as seen on the upper part of Figure 5-8. Similarly to procedure followed in Chapter 3 , the simulation $S_{11}$ parameter can be used to extract an "equivalent" circuit model. The equivalent model obtained for the probe in the middle of the array is seen in Figure 5-9. The same model used to individually characterize the cube probes is still applicable to the case of a cube probe in an array. 


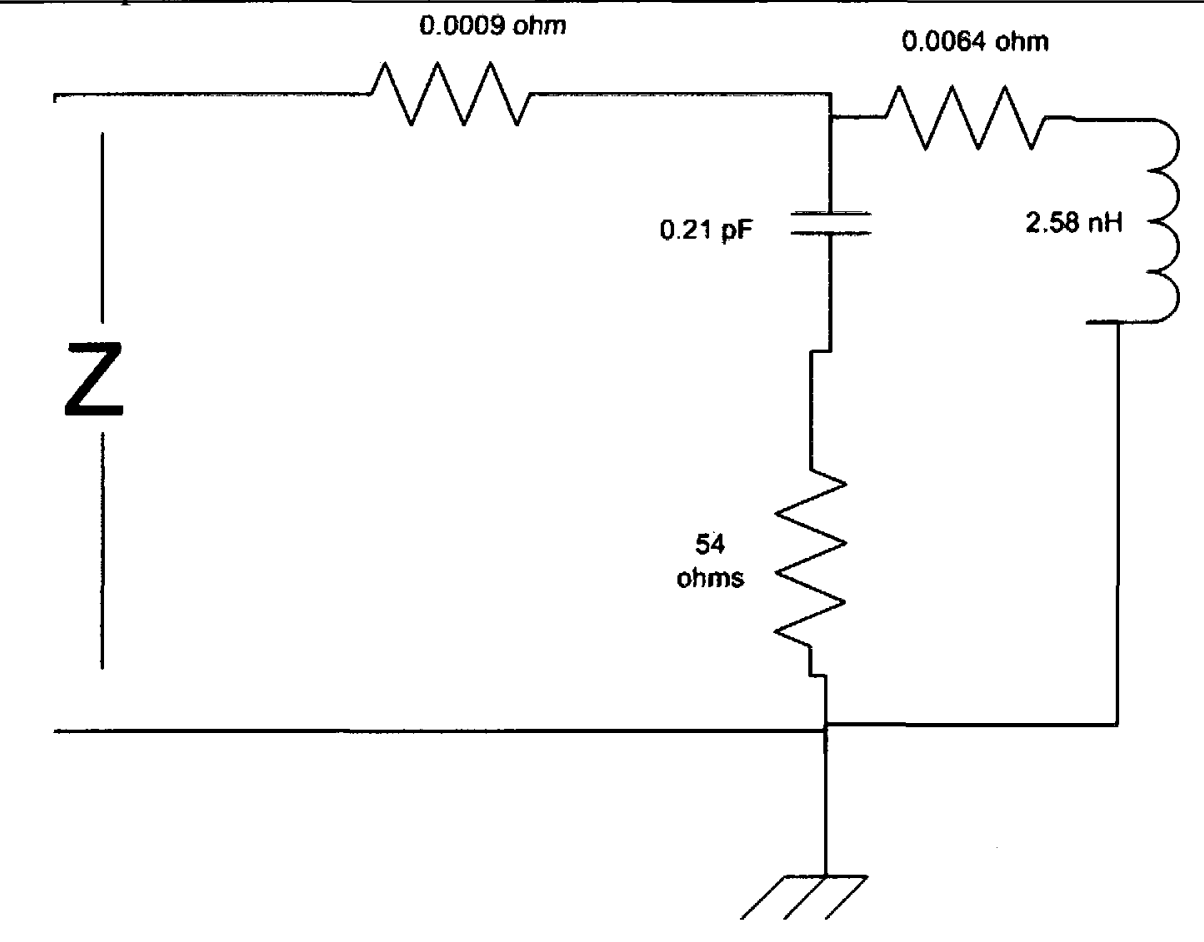

Figure 5-9: Equivalent model of a Cube Probe in the middle of an array of shorted similar probes

When comparing Figures 5-9 and 3-12, the equivalent models match closely. The element of most importance in the equivalent model is the inductance. In both models, the value of inductance is almost identical. Some discrepancy exists with the parasitic capacitances and the input resistances between both models. The discrepancy is due to the change in the structure causing different interaction of EM waves in the cube probe with the neighbouring probes.

To validate the simulation results, the same structure was built and measured in the lab. To access the middle cube probe, a surface mount RF SMB connector was utilized. A de-embedding process similar to the one undertaken in section 3.5.3 was done to remove the effect of the connector. Figure 5-10 shows the 
equivalent model of the cube probe in an array of shorted probes derived from measurements.

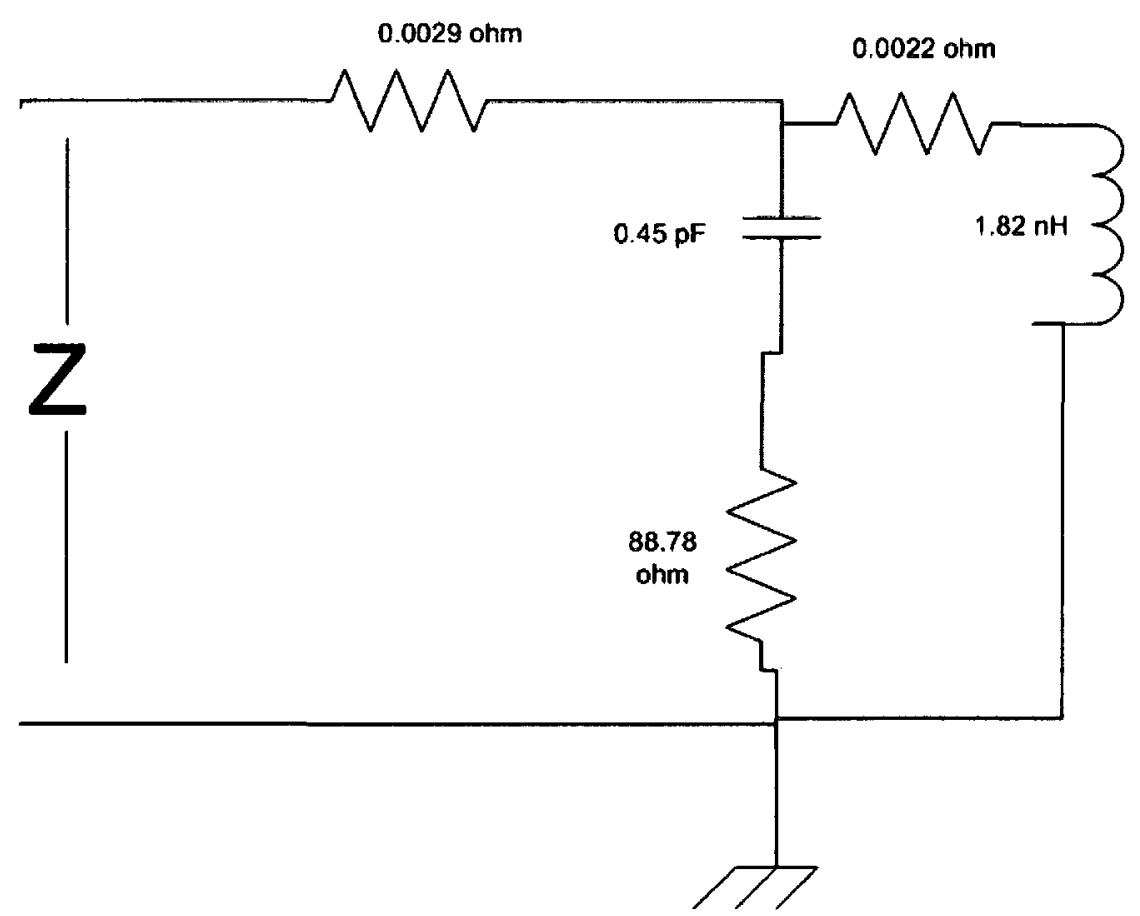

Figure 5-10: Measurement derived equivalent model of the cube probe in the middle of an array of similar shorted probes

The result obtained from the measurement shows some differences with simulations. First the inductance in the model is lower than that of the simulation. The parasitic capacitance is also higher in the measured model. One explanation for the discrepancy is the fact that the connector placed on the structure was tilted allowing for a layer of air to accumulate. Therefore, the de-embedding results are not as accurate as they should be. However, the equivalent model generated by the measurements does not show any major flaws but rather confirms the validity of the utilized model and the simulation results. 


\section{Cube Probe in an array of open circuited Cube Probes}

Similar to the simulation done previously for the array of shorted cube probes, a simulation was performed to determine the influence of neighbouring probes when they are open circuited. The simulated structure was that of the lower part of Figure 5-8. The computed equivalent model is shown in Figure 5-11.

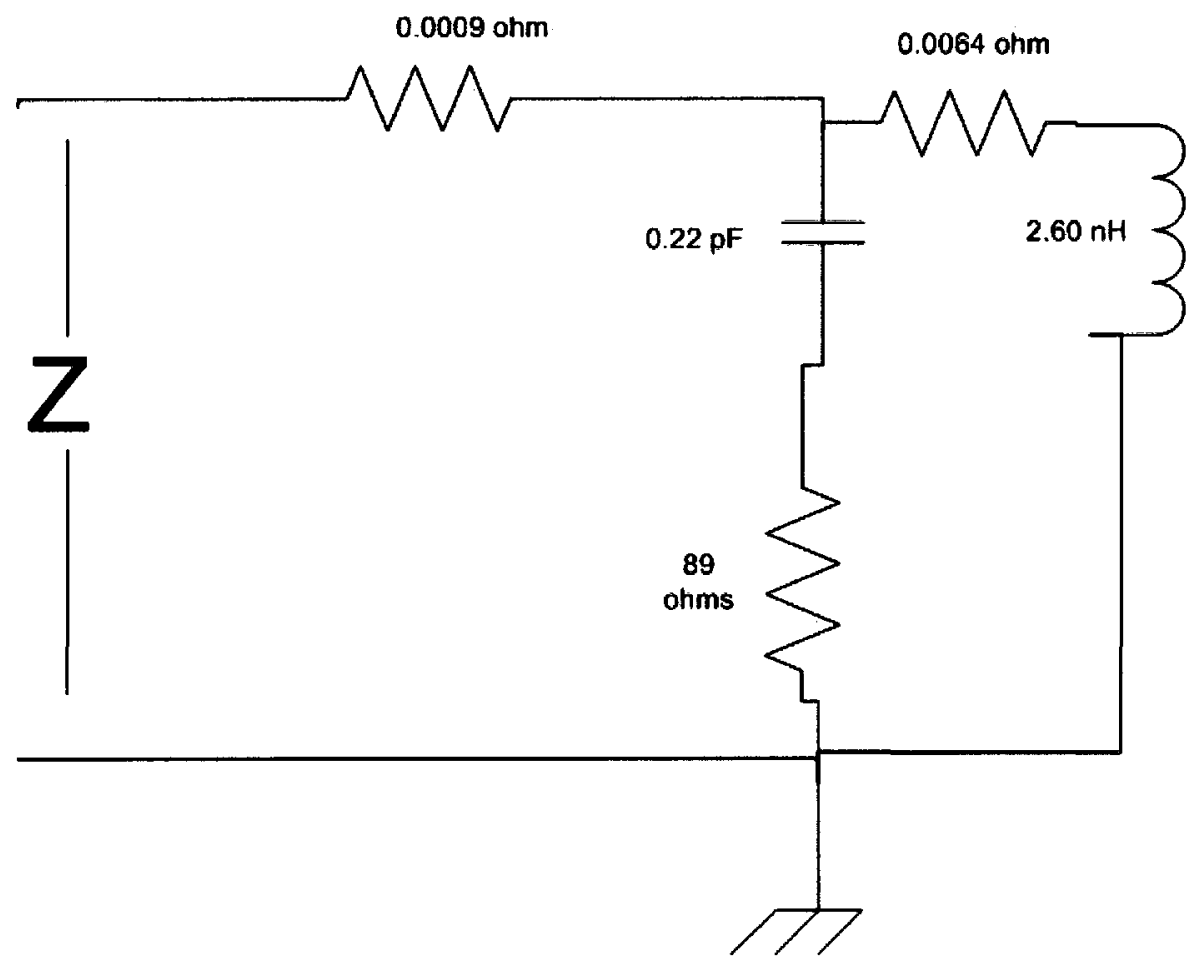

Figure 5-11: Equivalent model of a Cube Probe in the middle of an array of similar open circuited probes

Upon inspection of Figure 5-11 and by comparing it to Figure 5-9, one notices that the differences between both models are very minimal. The only actual difference is in the equivalent circuit's resistance. This result implies that it is slightly easier for the energy in the cube probe in the shorted array of probes to leak out. 
Assessment/Experimental Validation of LTCC SoP

An attempt at measuring the same structure failed due to adhesion issues with placing the surface mount RF connector. The first time the connector was placed incorrectly on the test structure. Therefore, upon its removal, it was difficult to remount it again while keeping the connector flat on the surface. For that reason, the de-embedding process would be very difficult and the results would not potentially show the actual input impedance of the structure. The measurement was abandoned. On the other hand, it was obvious from section 5.2.2 and from section 3.5.6 that there is a close match between the simulation results and the measurements. Therefore, the simulation results are sufficient in determining the influence of neighbouring probes on the cube probe under test.

\section{Cube probe in an array of $50 \mathrm{Ohm}$ terminated cube probes}

This third structure was not built but only simulated. The purpose of it was to create an environment where current flows in the cube probes in the array. However, that current would not be as high as the current in section 5.2.2 where the cube probes are shorted together.

Figure 5-12 shows the resulting equivalent model of the cube probe surrounded by probes that are terminated by 50 Ohms. The model compares very well with those shown in Figures 5-9 and 5-11. The differences in the results are very minimal. The inductance is of approximately $2.6 \mathrm{nH}$ and the parasitic capacitances are all in the order of $0.21 \mathrm{pF}$. 


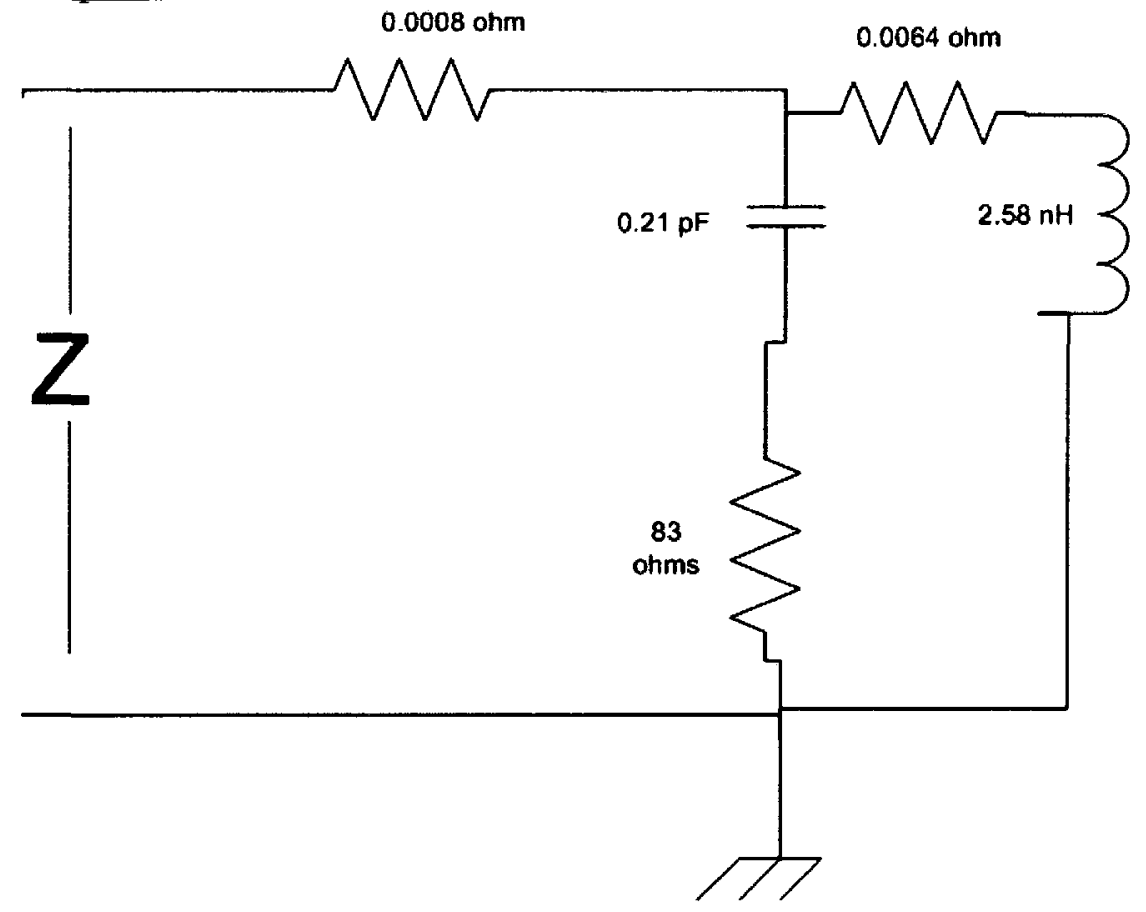

Figure 5-12: Equivalent model of the cube probe placed in the middle of an array of similar probes all terminated by $50 \mathrm{Ohms}$

\subsubsection{Summary of input impedance characterization}

In light of the simulation results and the measurement result, it can be said that the effect on the cube probe is not very significant from neighbouring elements. Although there is a small change in the equivalent model values, meaning that the resonant frequency of the probes might change slightly, the characteristic of the cube probe in its useful operating range does not change. The inductance of the cube probe in the array is around $2.6 \mathrm{nH}$ which is similar to its inductance in isolation. It should be noted that the inductance is the element of most importance as it represents the ability of the cube probe to collect EMI via mutual coupling to the DUT. Some minor variations occur with the other elements forming the equivalent models, but those have secondary effects. 
Assessment/Experimental Validation of LTCC SoP

Based on the above results, it may be said that the separation distance of $1 \mathrm{~mm}$ between the array elements does not significantly affect the behaviour of the cube probe. The cube probe remains inductive in the array and capable of acquiring magnetic field energy without interference from neighbouring probes. It should be noted that the impedance models obtained for each configuration could be exploited in subsequent SoP based modules.

\subsection{Package Characterization}

While the performance of the multiplexer chip and the cube probe in and outside of an array have been fully characterized, the characterization of interconnects in the LTCC package remains. Two main aspects are to be characterized. The first is the mutual coupling that exists between the interconnects given the small layout area in LTCC. This may ultimately affect the accuracy of the measured EMI data. The second aspect is the losses incurred through the interconnects between the cube probes and the multiplexer chips, and between the multiplexer chips themselves. This affects the calibration of the EMI diagnostic tool and also its sensitivity.

To characterize the package, it is sufficient to characterize roughly an eighth of the structure in Ansoft HFSS. Indeed, attempting to simulate the whole package leads to instrumental difficulties in the meshing for EM analysis. The total package was made of eight identical parts, with each part containing eight cube probes and a cavity for the placement of a multiplexer chip. Figure 5-13 displays the portion of the package that was simulated. 


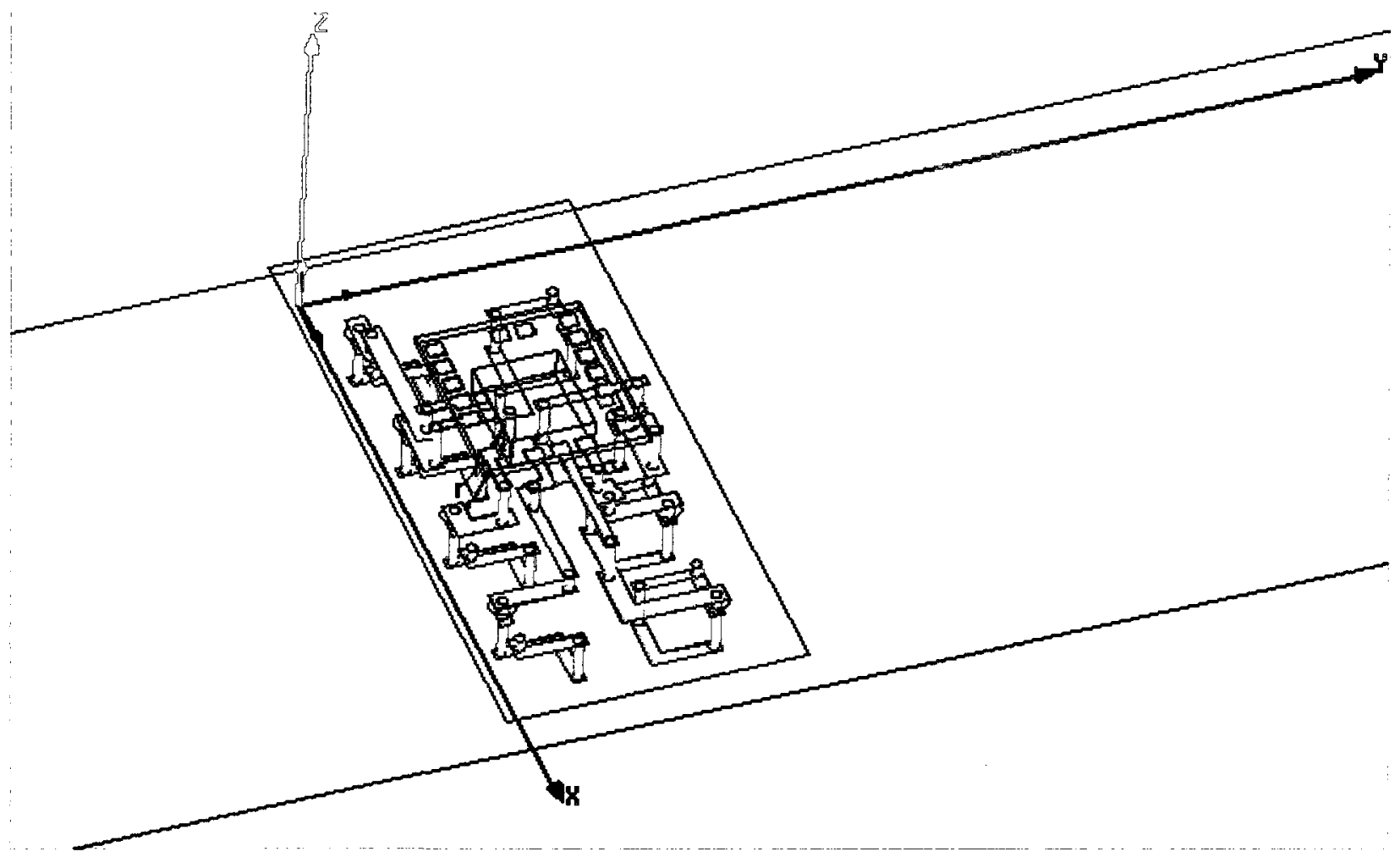

Figure 5-13: Structure Simulated for mutual coupling

\subsubsection{Mutual Coupling Simulations}

Although the characterization of the cube probe in an array was performed, the mutual coupling is still an important aspect to be assessed. Given the fact that the system on package contains 64 cube probes and nine multiplexer chips, the number of interconnects is large. Therefore, some simulations were performed to determine the mutual coupling starting with only two neighbouring cube probes. To do so, a lumped port was connected to the input of each cube probe being tested, and the $S_{21}$ was computed between the two ports, providing the signal transmission from one cube probe to the other. The simulations were then extended to find the effect of the interconnects on the mutual coupling. 
Assessment/Experimental Validation of LTCC SoP

Therefore, two lumped ports were placed at the multiplexer chip level representing two neighbouring $\mathrm{RF}$ input ports to the multiplexer chip. A similar $S_{21}$ simulation was performed to determine the change in mutual coupling, isolating the performance of the interconnects and the wire bonding.

\section{Mutual Coupling at the level of Cube Probes}

Although it was determined that the cube probe's impedance properties do not change significantly in an array, the mutual coupling between two neighbouring probes was simulated. Figure 5-14 shows the mutual coupling when the separation between two probes is $1 \mathrm{~mm}$. By inspecting the figure, it is clear that as the frequency becomes higher, the mutual coupling between neighbouring elements increases, dramatically. However, between $0.1 \mathrm{GHz}$ and $3 \mathrm{GHz}$ the mutual coupling is very low (below $-20 \mathrm{~dB}$ ). Therefore, the array concept, having neighbouring cube probes with a separation of $1 \mathrm{~mm}$, is suitable for the EMI frequencies targeted in this work.

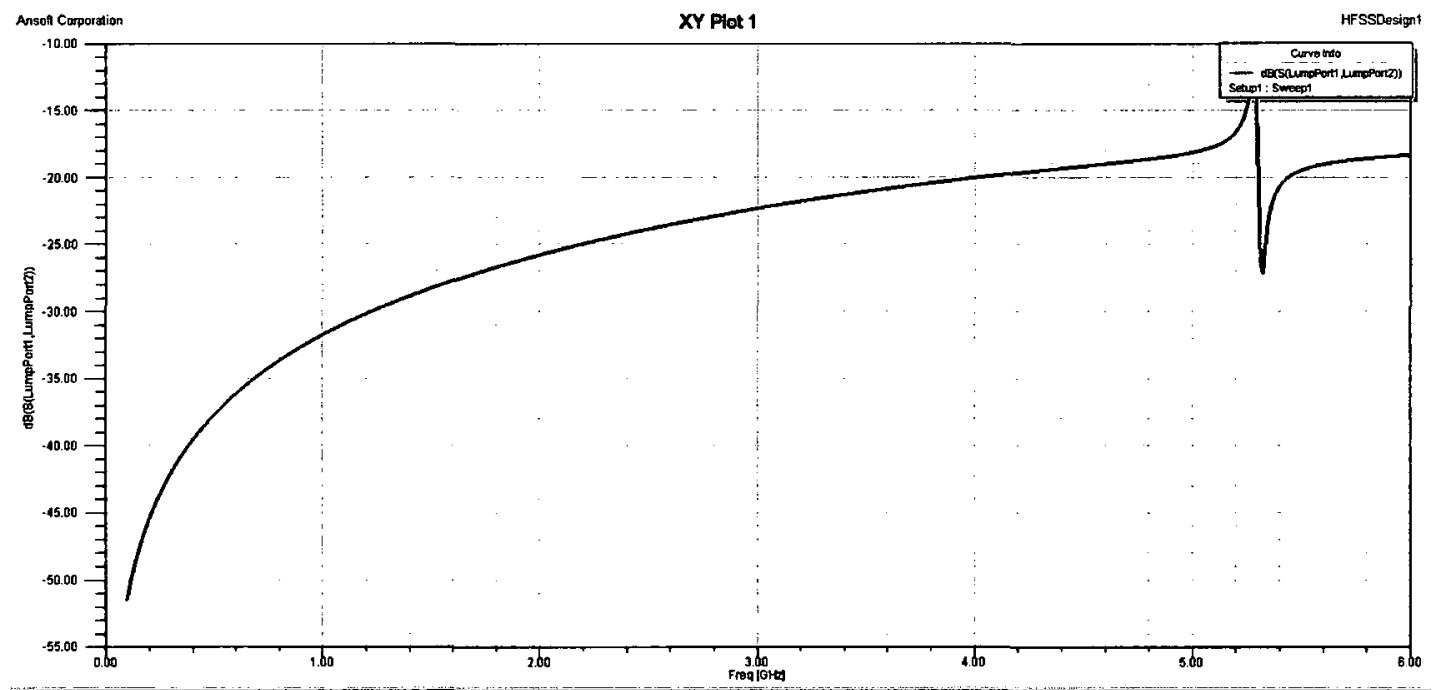

Figure 5-14: Mutual Coupling between two neighbouring Cube Probes separated by $1 \mathrm{~mm}$. 


\section{Mutual Coupling at the level of the multiplexer chip}

After the interconnects were added between the cube probes and the multiplexer chip, a similar simulation was undertaken as the one mentioned just previously. Two lumped ports were placed at the RF inputs of the multiplexer chip and an $S_{21}$ simulation was performed to determine the mutual coupling between the two ports. Figure 5-15 shows the mutual coupling levels obtained at the input to the multiplexer chip.

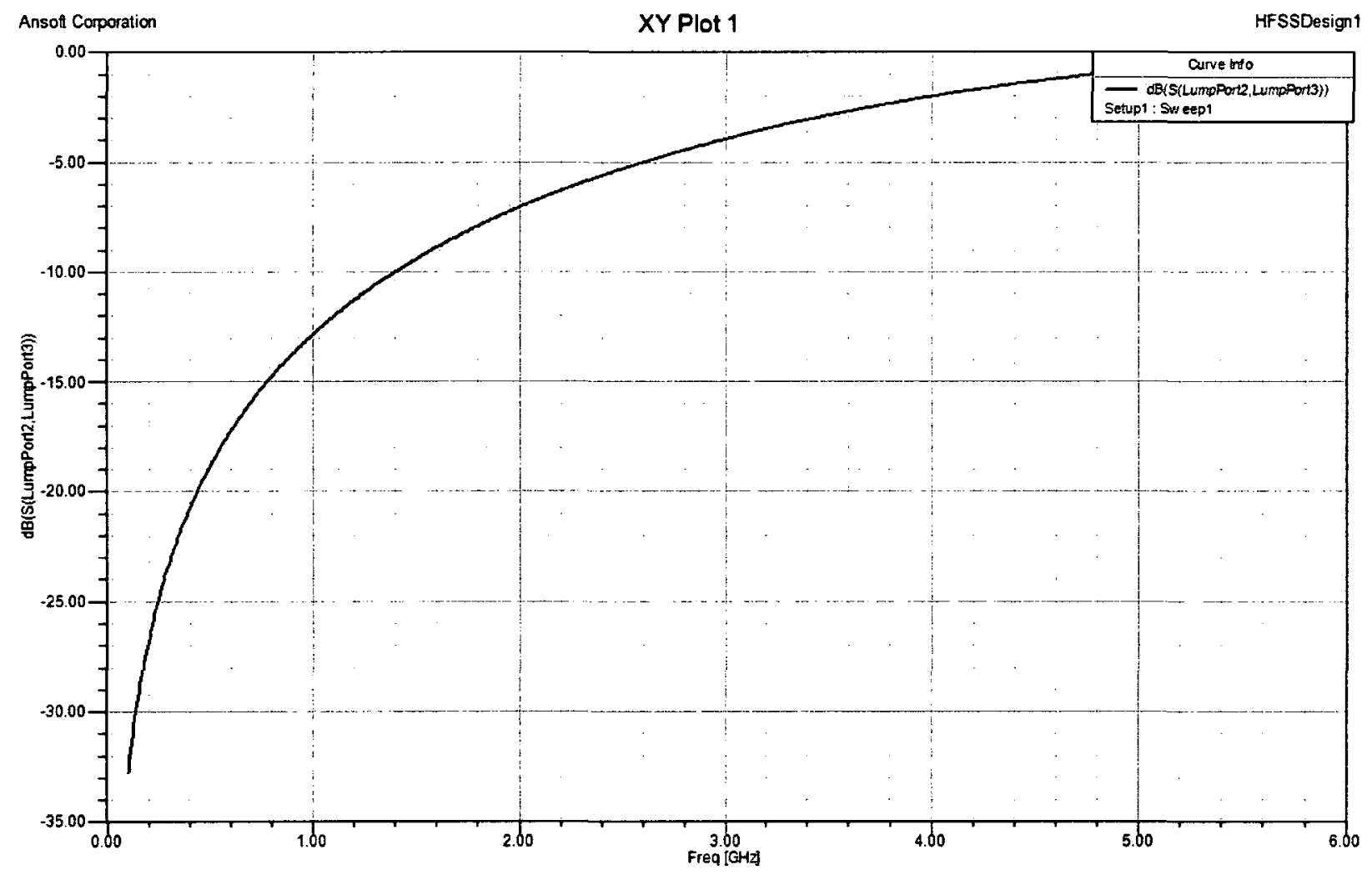

Figure 5-15: mutual coupling at the input ports of a multiplexer chip.

Based on the results in this figure, any operation beyond $500 \mathrm{MHz}$ seems problematic since the mutual coupling is significant beyond that point, reaching $4 \mathrm{~dB}$ at $3 \mathrm{GHz}$. Therefore, in order to reduce the mutual coupling and to make 
efficient use of the interconnects in the package, a ground plane was introduced.

The ground plane was located two layers above the array of cube probes. Figure 5-16 shows the ground plane and the via holes created to pass the signal from the cube probes to the multiplexer chip.

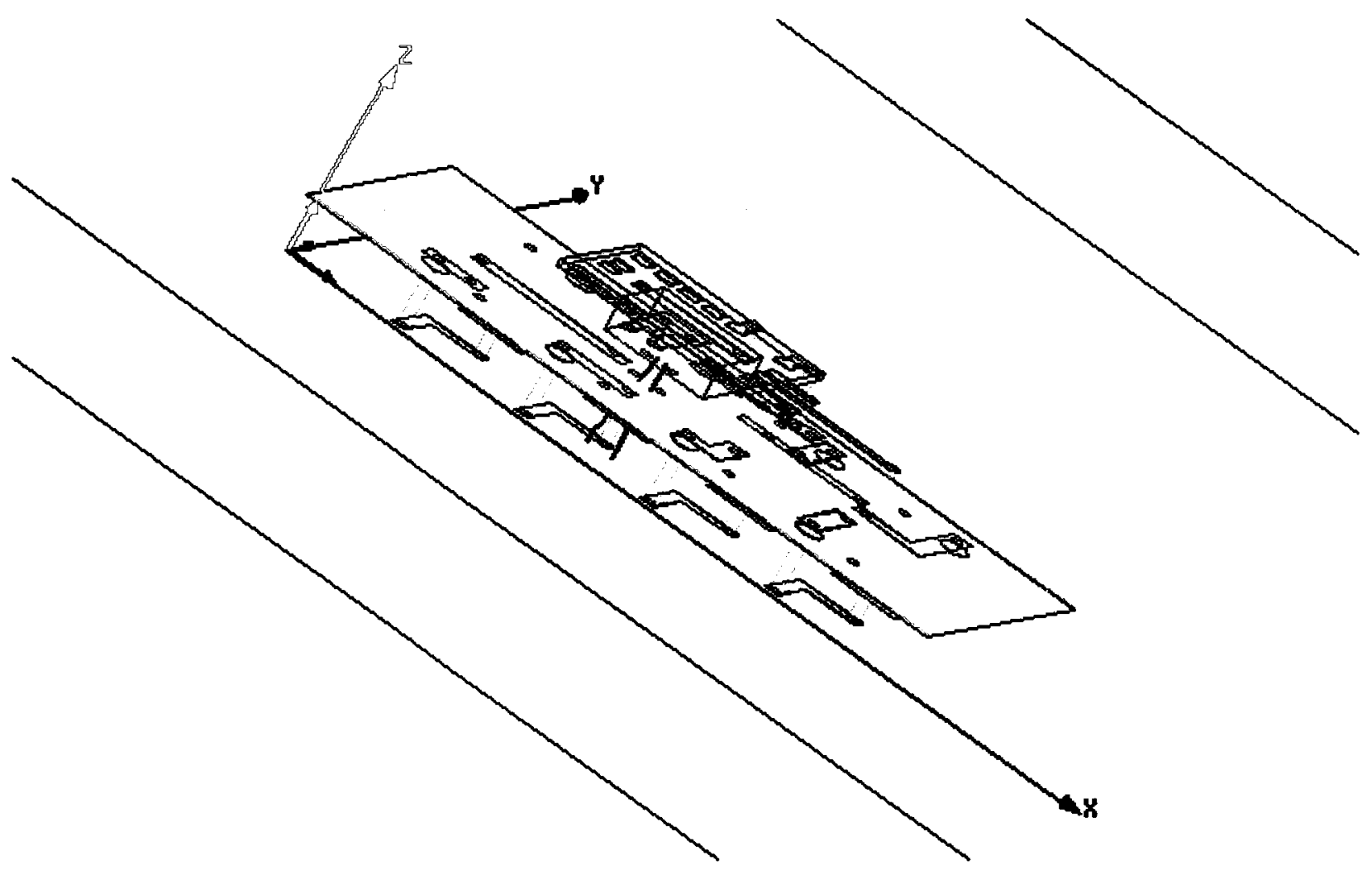

Figure 5-16: The simulated structure for mutual coupling with the inserted ground plane in colour.

The main purpose of the ground plane was to reduce mutual coupling between interconnects. Figure 5-17 shows the mutual coupling after the introduction of the ground plane. It should be noted that the ground plane required through-holes to pass the cube probe signal into the rest of the package and to the multiplexer chips. 


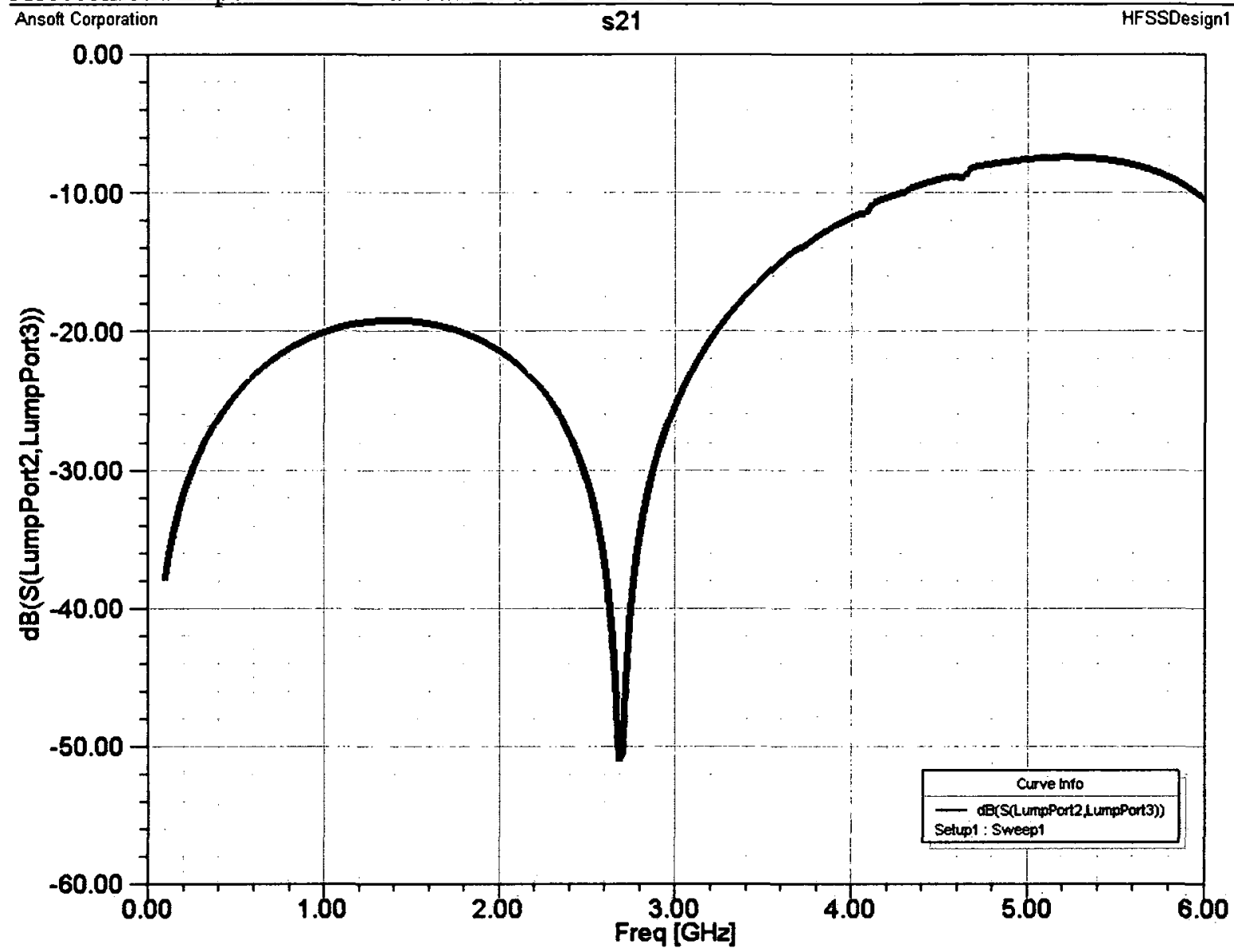

Figure 5-17: Mutual Coupling at the multiplexer chip level after the introduction of the ground plane.

Upon inspection of Figure 5-17, it was clear that the mutual coupling was significantly reduced after the introduction of the ground plane two layers above the Cube Probes in the system on package SoP. For the most part between 0.1 and $3 \mathrm{GHz}$, the mutual coupling remained below $-20 \mathrm{~dB}$. Therefore, the interconnect network was made suitable for the array-based EMI pickup application.

Although the result in Figure 5-17 was considered acceptable, an attempt was made to further reduce the mutual coupling levels at higher frequencies. To 
accomplish this, via fences and ground planes were introduced between the interconnects. The isolation between the interconnects was enhanced. Figure 518 shows the mutual coupling at the input of the multiplexer chip once the via fences were introduced. It can be seen that the maximum coupling of $-7 \mathrm{~dB}$ obtained at $5 \mathrm{GHz}$ is reduced to below $-20 \mathrm{~dB}$.

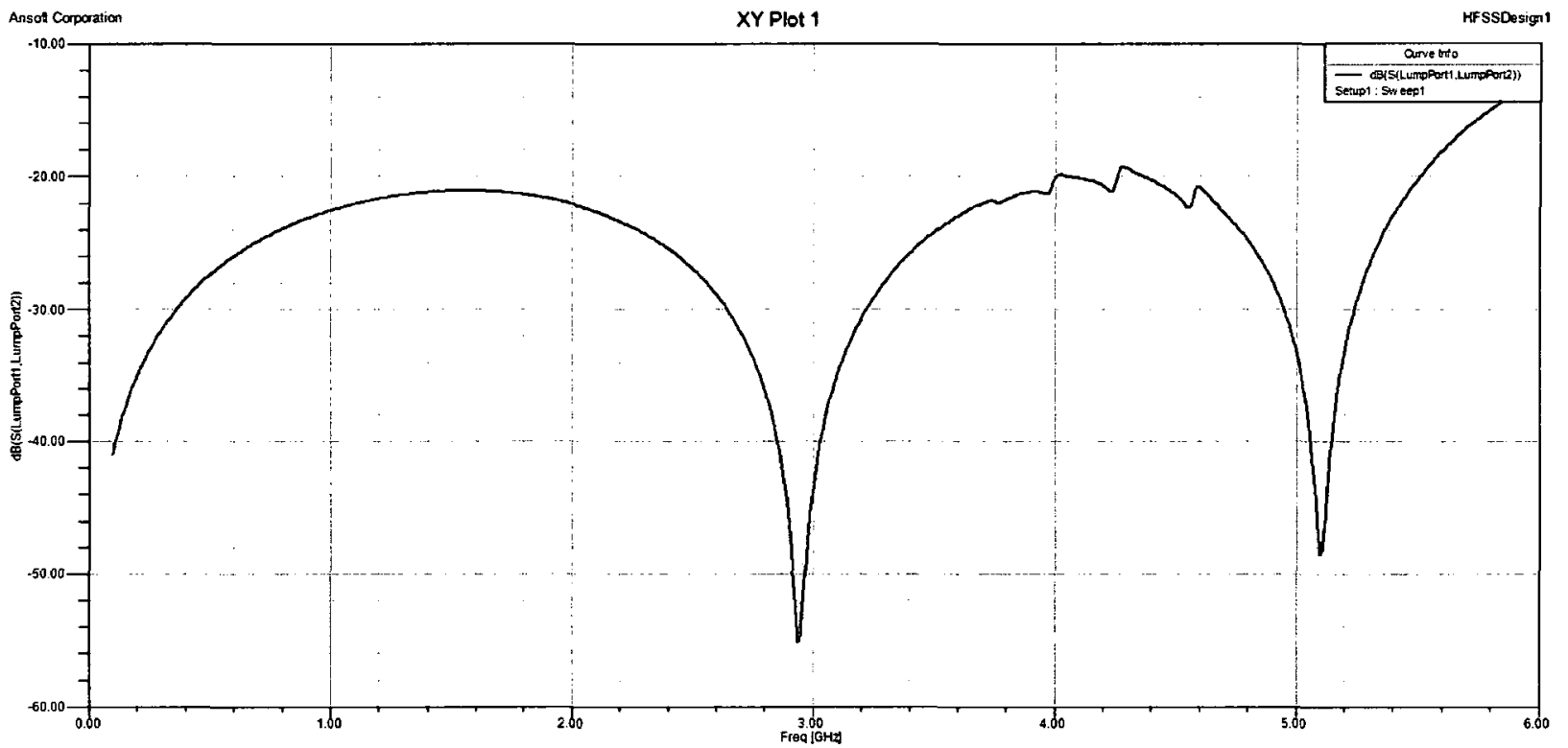

Figure 5-18: Mutual Coupling at the multiplexer chip level after the introduction of ground planes and via fences to isolate interconnects.

Therefore, according to Figure $5-18$, it is possible to create an environment where the mutual coupling is greatly reduced at higher frequencies by introducing via fences and more ground planes to isolate the interconnects. However, since the present application of the EMI sensor is to work below $3 \mathrm{GHz}$, the latter option was abandoned. When the package was fabricated, only one ground plane was introduced two layers above the cube probes to reduce the mutual coupling in the 0.1 to $3 \mathrm{GHz}$ band. 


\subsubsection{Loss Characterization}

The other parameter of interest in the characterization of the LTCC package is the losses incurred in the transmission lines. Therefore, a simulation was performed to find the losses in the transmission lines connecting the cube probes to the multiplexer chip. Furthermore, another simulation was conducted to find the losses in the transmission lines connecting the peripheral multiplexers to the ninth multiplexer. Figures 5-19 and 5-20 show the losses in the transmission lines between the cube probes and the multiplexers on the periphery and between the peripheral multiplexers to the ninth multiplexer respectively.

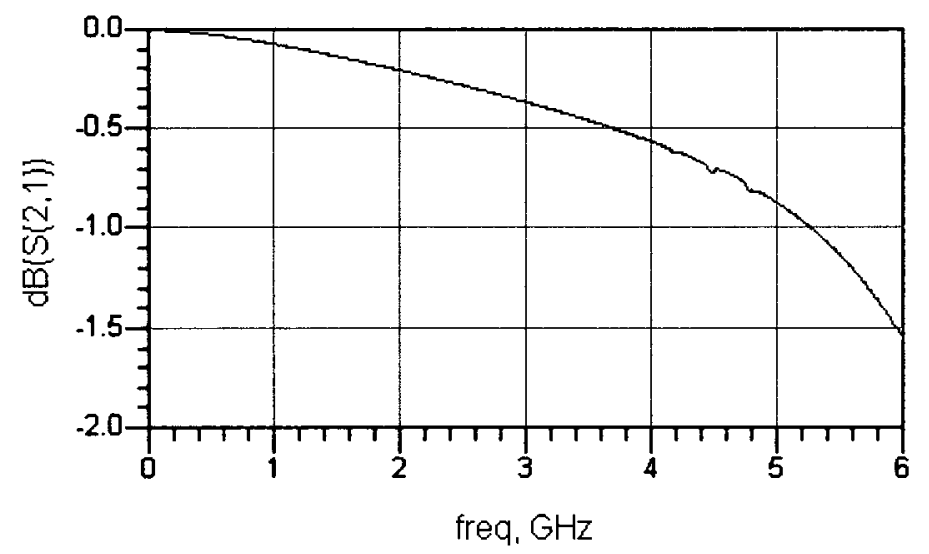

Figure 5-19: Insertion loss of the transmission line from the Cube Probe to the multiplexer chip 


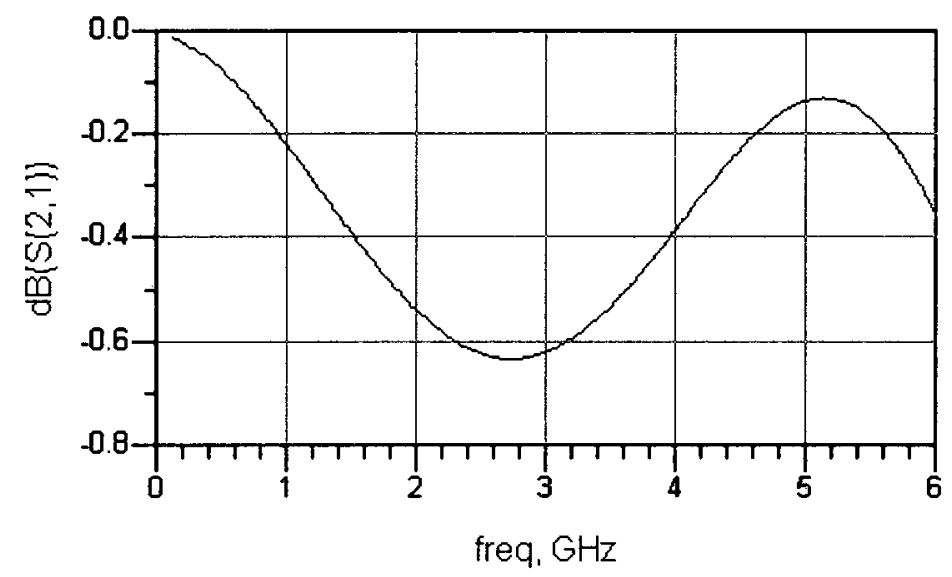

Figure 5-20: Insertion loss of the transmission line from a peripheral chip to the ninth multiplexer chip

As it is expected in an LTCC package, the transmission losses are not important due its favourable microwave properties. The losses in Figure 5-20 show an oscillatory behaviour due to the longer length of line $(2 \mathrm{~cm})$ compared to that in Figure 5-19 $(5 \mathrm{~mm})$. In the band between 0.1 and $3 \mathrm{GHz}$, the maximum amount of loss between a cube probe and the ninth multiplexer chip (which connects to the output of the system) is approximately $1 \mathrm{~dB}$.

\subsection{Assembly of the SoP}

The initial task involved in the assembly of the SoP was the placement of the multiplexer chips and their wire bonding to the LTCC substrate. Following this, the DC connections necessary for biasing the chips and the multiplexer control lines were made. The connection to the control lines was treated like a DC 
Assessment/Experimental Validation of LTCC SoP

connection due to the relatively slow switching speed associated with EMI testing.

The assembly of the SoP was not an easy task given the small size pads under consideration. To be able to interface the SoP to the test equipment, a special PCB with a hole in the middle was developed. The hole was created to glue the SoP from the bottom to the $\mathrm{PCB}$, eliminating interference from any voltage supply lines or RF connections with the cube probes. On the PCB, voltage lines were soldered so as to connect to the voltage sources, and to the various pads on the SoP. Some of the designed pads were as small as $0.5 \mathrm{~mm}$ by $0.5 \mathrm{~mm}$, which are very small for hand-made connections. This problem was not foreseen when the SoP was being designed and for future prototypes, DC connection pads should be made larger.

\subsection{SoP Testing}

Although the elements forming the SoP were tested individually, it was important to test the total system (as shown in Figure 4-15) in the active mode. To fully validate the system, the EMI emitted from a microstrip line was to be tested. The EMI was to be measured by each cube probe and recorded to form a mapping of EMI emissions emanating from the microstrip line. Table 5-1 displays the codes responsible for the detection of the signal picked up by the cube probe in the specific location on the array. For example, when the input to the SoP is the code 000000 , the $4^{\text {th }}$ cube probe from the left on the top line is selected, and its 
Assessment/Experimental Validation of LTCC SoP

captured magnetic field intensity is transferred to the input of the first multiplexer

chip and then to the ninth multiplexer chip to finally reach the output of the SoP.

\begin{tabular}{|l|l|l|l|l|l|l|l|}
\hline 000101 & 000001 & 000100 & 000000 & 111111 & 111011 & 111110 & 111010 \\
\hline 000010 & 000110 & 000011 & 000111 & 111000 & 111100 & 111001 & 111101 \\
\hline 100101 & 100001 & 100100 & 100000 & 011111 & 011011 & 011110 & 011010 \\
\hline 100010 & 100110 & 100011 & 100111 & 011000 & 011100 & 011001 & 011101 \\
\hline 001101 & 001001 & 001100 & 001000 & 110111 & 110011 & 110110 & 110010 \\
\hline 001010 & 001110 & 001011 & 001111 & 110000 & 110100 & 110001 & 110101 \\
\hline 101101 & 101001 & 101100 & 101000 & 010111 & 010011 & 010110 & 010010 \\
\hline 101010 & 101110 & 101011 & 10111 & 010000 & 010100 & 010001 & 010101 \\
\hline
\end{tabular}

Table 5-1: voltage control codes representing activated the Cube Probe and its position in the array

An attempt was made to use a microstrip line as the device under test in a similar configuration to the one shown in Figure 3-6. For that purpose, the microstrip line was fed at one end by a signal generator having a frequency of $1 \mathrm{GHz}$. The second end of the microstrip line was kept open to maximize reflections on the line and increase the emitted magnetic field at the locations of the current maxima. The SoP was placed above the microstrip line, and the control voltages were varied to obtain the magnetic field measured by the various cube probes. The output of the SoP was connected via a worm cable to a spectrum analyzer to observe the level of signal measured. While switching the control voltages, it was noticed that the change appearing on the spectrum analyzer was very minimal, and almost negligible.

Another test was then undertaken by applying the signal straight to the queried cube probe by means of the tip of an SMA cable acting as a localized antenna. While keeping the control bits the same, it was found that by changing the position of the antenna below the cube probes over the complete surface of the 
Assessment/Experimental Validation of LTCC SoP

SoP, a maximum difference of $5 \mathrm{~dB}$ in the signal level was observed on the spectrum analyzer. This suggests a limited ability of the SoP to detect EMI signals over a large range. However, the signal levels employed were very close to the noise floor of the spectrum analyzer and this may explain the poor dynamic range. Also the EMI source may not have been adequate.

To determine whether the switching was occurring on the multiplexer level, two tones were then utilized to inject two signals at $850 \mathrm{MHz}$ and $880 \mathrm{MHz}$. The tones were applied using the tips of SMA cables beneath two distinct cube probes. The chosen cube probes were selected by the control bits of 100101 and 011101. The frequencies of both tones had to be very close so that the effect of propagation loss would be the same for both signals, since loss varies with frequency. While switching the control bits corresponding to the selected cube probes, the spectrum output was observed. It was found that when the desired cube probe is selected via the control bits, its output was superior to that of the signal fed to the unselected cube probe. Figures $5-21$ and $5-22$ show the output spectrum with markers displaying the power levels of the measured signals for the first test when a signal of $850 \mathrm{MHz}$ was applied below the selected cube probe. In Figure 5-21, the output from the selected cube probe measured -63 $\mathrm{dBm}$, while the output from the non-selected cube probe measured $-68.33 \mathrm{dBm}$ in Figure 5-22. In Figures 5-23 and 5-24, the control bits were switched to reverse the selection such that the desired cube probe had an applied signal at $880 \mathrm{MHz}$. Thus the selected cube probe picked up the signal at $880 \mathrm{MHz}$ and the 
Assessment/Experimental Validation of LTCC SoP

recorded level was of $-62.83 \mathrm{dBm}$. The non-selected cube probe picked up a signal at $850 \mathrm{MHz}$ and recorded a level of $-66.53 \mathrm{dBm}$.

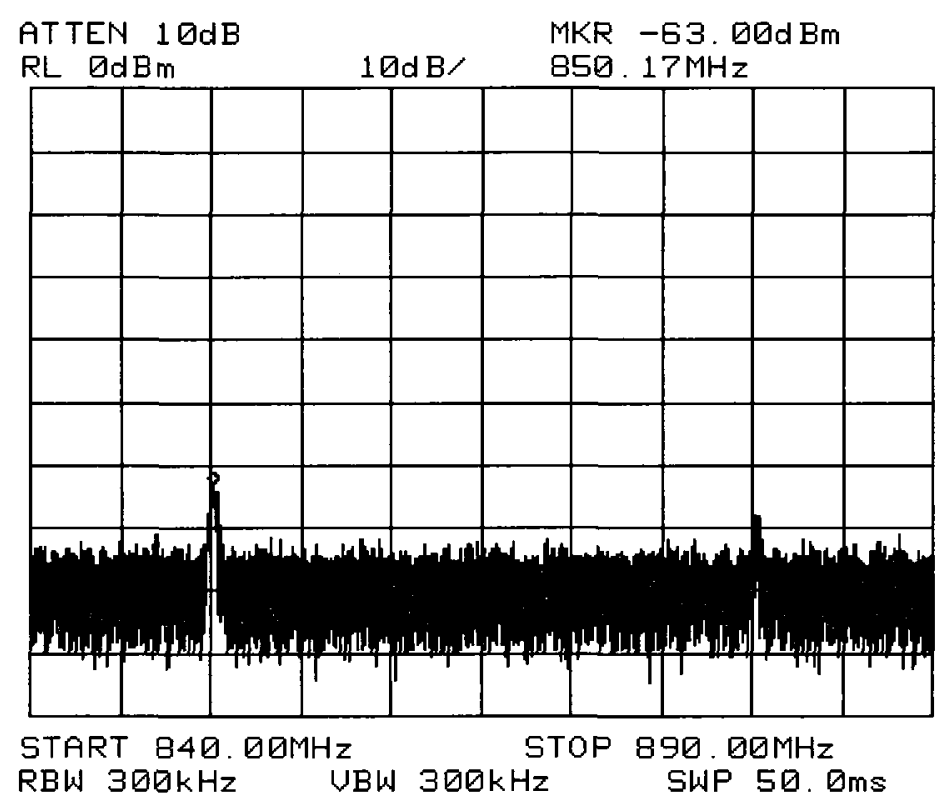

Figure 5-21: Spectrum analyzer output marking the signal level at $850 \mathrm{MHz}$ for the first test

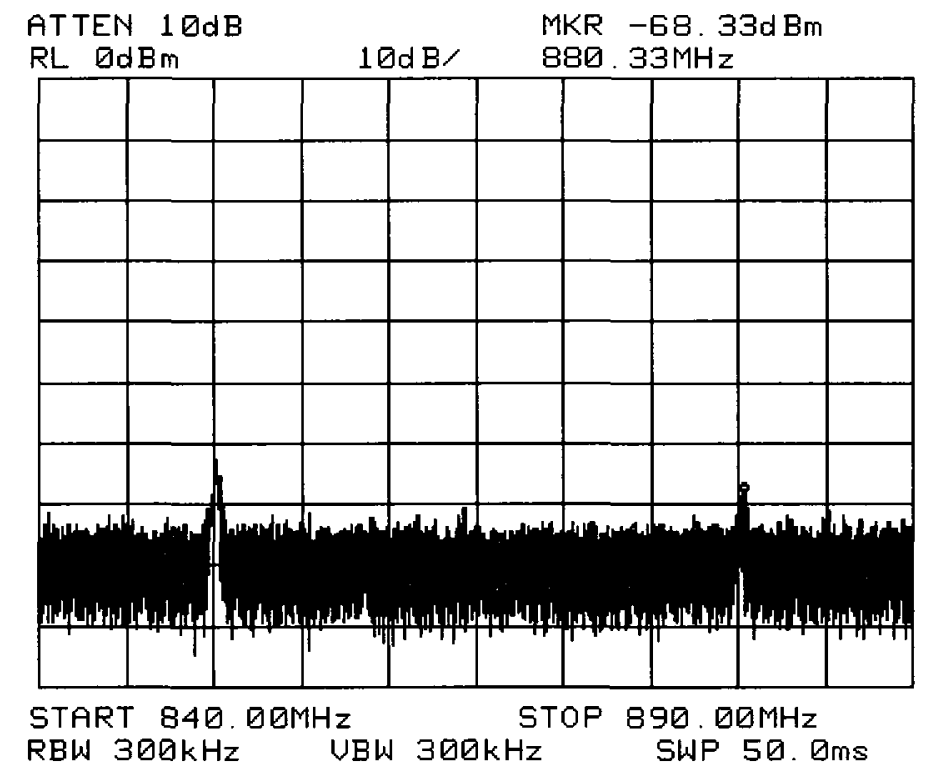

Figure 5-22: Spectrum analyzer output marking the signal level at $880 \mathrm{MHz}$ for the first test 


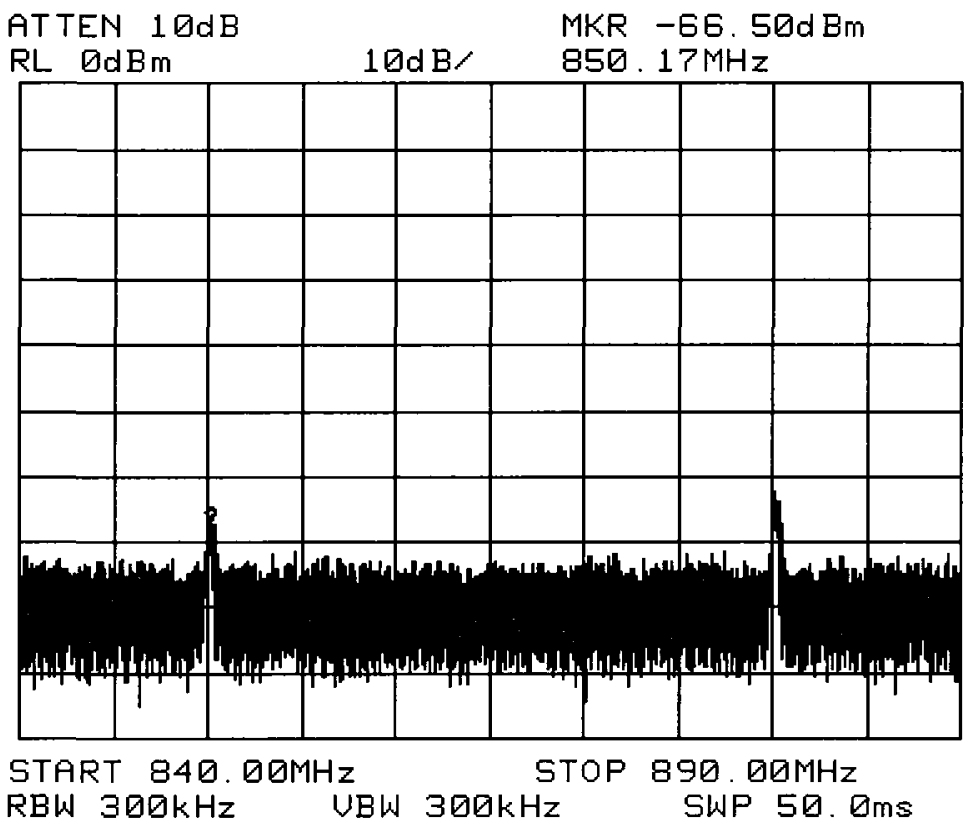

Figure 5-23: Spectrum analyzer output marking the signal level at $850 \mathrm{MHz}$ for the second test

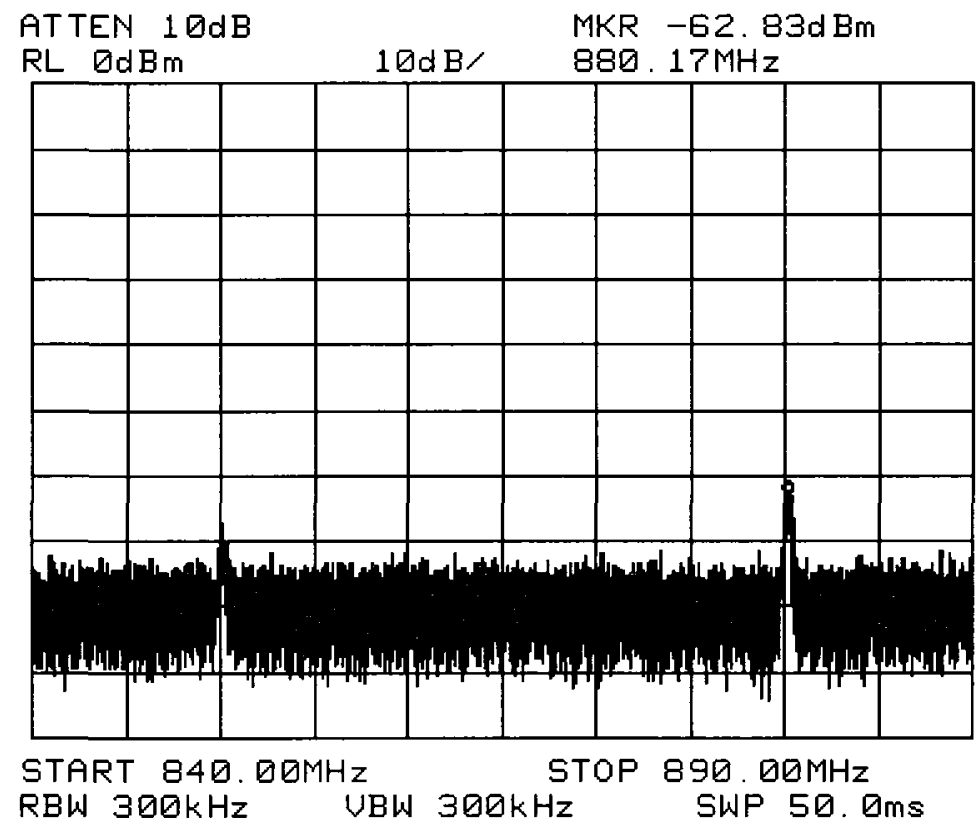

Figure 5-24: Spectrum analyzer output marking the signal level at $880 \mathrm{MHz}$ for the second test 
Assessment/Experimental Validation of LTCC SoP

Based on the test results, it was clear that the switching was working in the system. The difference in the levels on the other hand was very low, varying between 3.5 to $5 \mathrm{~dB}$. The limited difference would not be suitable for the system to be able to characterize the EMI emissions of a test structure.

\subsection{Summary of Assessment and Discussion}

In this chapter, all the different blocks that form the system on package SoP were tested individually and collectively. The multiplexer chip was characterized, and it was found that its gain had dropped sharply due to a design flaw. When the design flaw was corrected, it was found that the gain of the chip improved by 25 to $30 \mathrm{~dB}$.

Furthermore, the cube probe was characterized in three different array configurations. All three arrays contained nine probe elements, and the middle probe of the array was the probe under test. Its input impedance was examined when all the peripheral cube probes were shorted to ground, open-circuited, or connected to 50 Ohms. The input impedance stayed relatively unchanged for all three conditions. Thus, the effect of the neighbouring probes on a cube probe in an array remains negligible for a spacing of $1 \mathrm{~mm}$ between probes.

In addition, mutual coupling was simulated. The transmission lines or the interconnects connecting the cube probe to the multiplexer chips were found suitable at the frequencies of operation because of their relatively low mutual coupling. The losses in the transmission lines were also simulated and found to be low. 
Assessment/Experimental Validation of LTCC SoP

Finally, the active testing of the full system was performed. Although the results were not as favourable as when the system was tested in parts, the overall concept of the multiplexer switched LTCC probe array was shown to be operational. Due to the losses in the multiplexer chips, it was found that the output signals were weak and close to the noise levels, making it difficult to detect EMI.

Based on the above, simulations were performed in HP-ADS at a system level to determine the overall response when the multiplexer chips were working well and when they were not. For that purpose, S-parameters files of the various system blocks were utilized from prior simulations in Ansoft HFSS and Cadence Design Tools. The simulation was to determine the level of the output signal when a cube probe from the SoP is placed at $1 \mathrm{~mm}$ above a $50 \mathrm{ohm}$ microstrip line and measuring its EMI.

Figures 5-25 and 5-26 display the difference in the output signal levels when the multiplexer chips are corrected (as described in section 5.1.2) and are working properly. The difference in the output levels observed in Figures 5-25 and 5-26 exceed $70 \mathrm{dBs}$ for some frequencies. Although these results are found by simulation of the cube probe pickup and the propagation of the signal within the SoP, the difference between the output signal levels is independent of any differences that might occur with the measurements. Therefore, based on these results, it can be concluded that a minor change to the output buffer of the multiplexer chip can render the system operational in the active mode. It then 

to the precise characterization of circuit-level EMI.

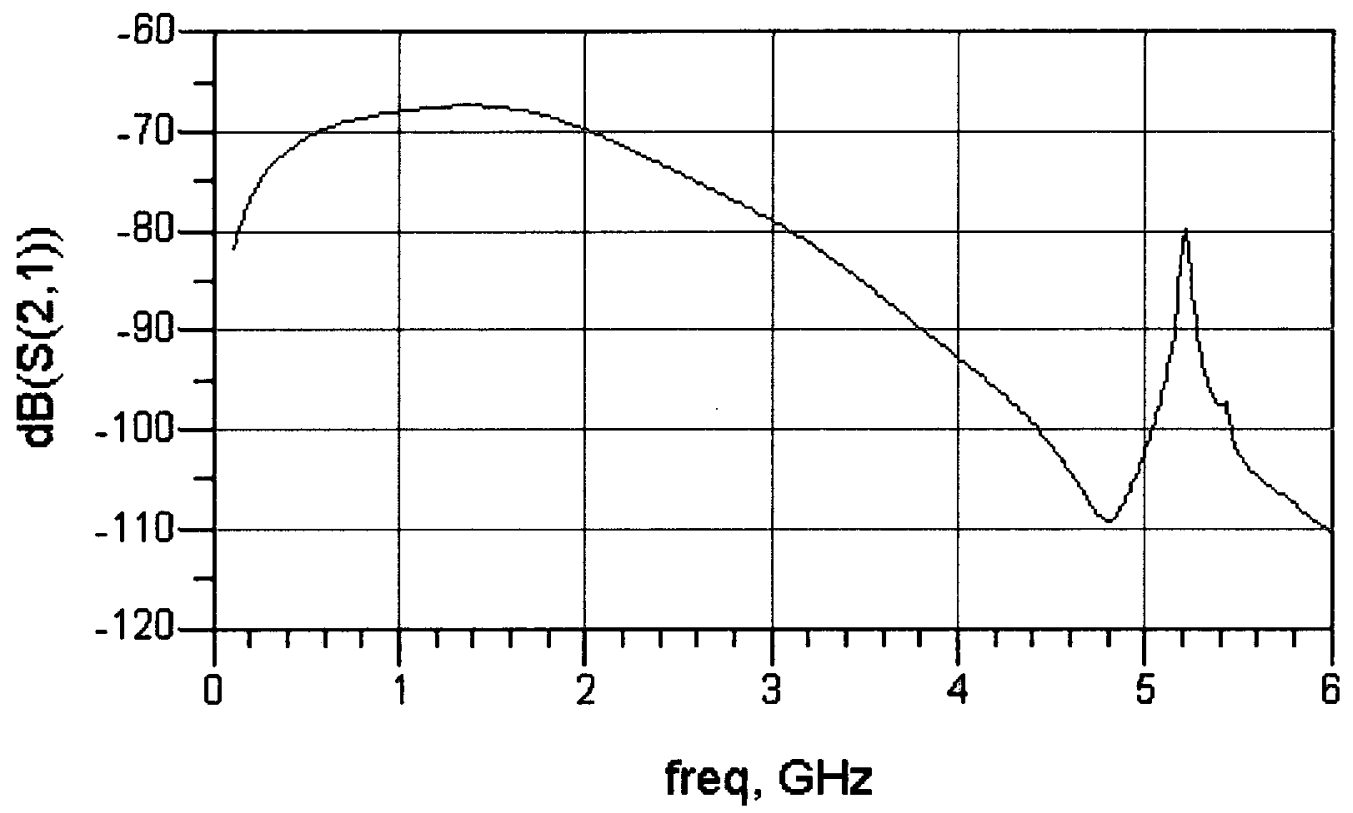

Figure 5-25: Insertion loss of the system measuring EMI at $1 \mathrm{~mm}$ over a microstrip line when the output buffer of the chip is not working 


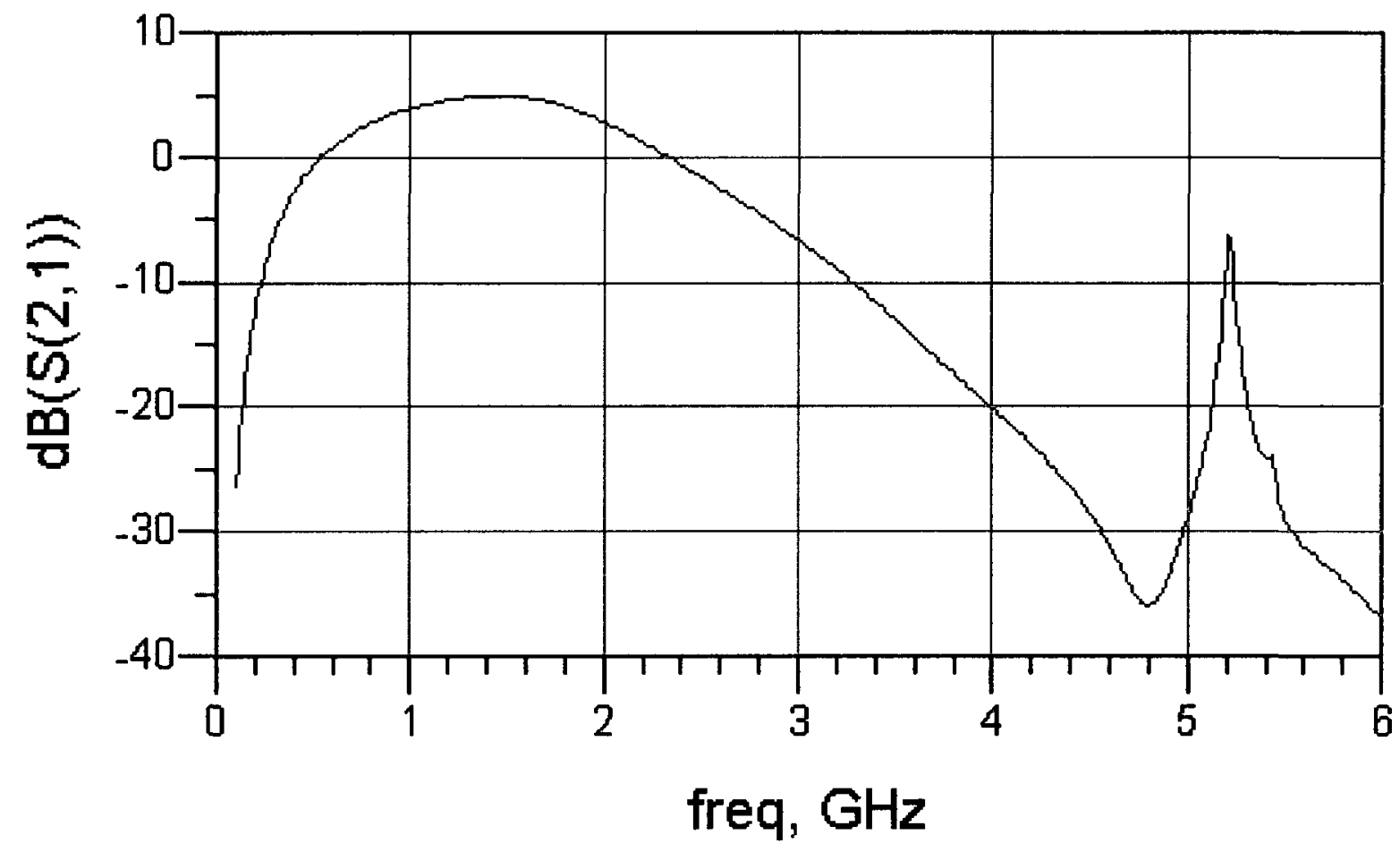

Figure 5-26: Insertion loss of the system EMI at $1 \mathrm{~mm}$ over a microstrip line when the output buffer is working 


\section{Chapter 6}

\section{Conclusions and Future Work}

\subsection{Conclusions}

Novel EMI measuring probes have been investigated in this thesis. With these probes, it is possible to have them integrated in printed circuit boards. Therefore, active elements can be grouped with these probes to process the data gathered by the probes. Four different probe variations were explored: the cube probe, the tangential probe, the normal probe, and the double cube probe.

The input impedances of all the four elements were found and their ability to pick up EMI from a matched 50 Ohm microstrip line was measured. The cube probe and the double cube probe were found fit to sense magnetic near fields in all the three spatial dimensions. Given this result, the cube probe and the double cube probe are considered to be ideal for sensing magnetic fields emanating from circuits without the need for a change in the orientation of the probes.

Traditional EMI characterization involves the use of scan tables to displace a magnetic field probe over a circuit under test. With the new integrated probes, the idea of having an array of EMI sensing probes in a system on package SoP was explored to eliminate the need for scan tables. Since LTCC provides an excellent packaging medium for 3D packaging and for systems on package, the cube probes and the double cube probes were characterized in LTCC, and in Ferrite LTCC for enhanced sensitivity. The cube probe was characterized 
Conclusion

110

through simulation and measurements as a stand-alone element in LTCC. Then

the cube probe was characterized in an array of similar elements to determine the effects the neighbouring probes have on the probe under test. The distance between the cube probes in the array was $1 \mathrm{~mm}$. The input impedance of the cube probe in the array was simulated for the conditions when all the other cube probes were shorted to ground, when all were open circuited, and when all were terminated with 50 Ohms. The input impedance was measured as well for the case when all the neighbouring cube probes were shorted to ground. It was found that the surrounding array elements had minimal effect on the performance of the cube probe.

A multiplexer was designed and fabricated in $0.13 \mu \mathrm{m}$ CMOS technology from IBM. The purpose of the multiplexer was to serve as a means to select a particular input from a cube probe in the array. A system of 64 cube probes in an array (with $1 \mathrm{~mm}$ spacing between elements) and nine multiplexers was then fabricated in LTCC. With a 6 digit binary code, each array element could be accessed and its sensed level stored to form a mapping of EMI emissions from a circuit under test.

Due to a malfunction in the output buffer of the multiplexer chip, the EMI diagnostic system was not fully characterized. However, it was shown that the concept of switching worked well in the multiplexers, allowing for a future implementation to operate with minor changes to the multiplexer chip. When the output buffers on the multiplexer chip are assumed to be functional, a gain of 
Conclusion

111

more than $60 \mathrm{dBs}$ is observed, rendering the dynamic range of the system very high.

Therefore, it can be concluded that a system on package implementation carrying an array of cube probes and active circuits can fully replace the work done by the scan table. This new alternative for EMI testing is cost effective, since scan tables are expensive to own and maintain. Furthermore, this new alternative could allow for much quicker characterization of EMI.

\subsection{Future Work}

In light of the results obtained during the assessment of the designs, it is clear that another attempt at ensuring a total functionality of the SoP is needed. For that reason, a correction of the multiplexer chip should be pursued to ensure that the output buffer is not contributing loss but rather gain.

Furthermore, a calibration method should be implemented to calibrate the difference in the paths loss for each cube probe. One way to implement such a calibration is to line up rows of cube probes in the array over a matched microstrip line. Knowing that the measured EMI by all the cube probes should be identical, the system can be calibrated to measure other circuitry by accounting for the difference in the sensitivity measured by each cube probe. Also, the individual cube probes could be calibrated with reference to a known $\mathrm{H}$-field source so that absolute EMI measurements can be made.

A new implementation is also needed for the SoP. An extra digital bit or more should be included in the selection of the cube probes to disable the multiplexer 
Conclusion

chips that are not needed. On the fabricated SoP, the 6 bits utilized were enabling the peripheral multiplexers while one only should have been enabled. Controlling the disabling and enabling of the peripheral multiplexers will lead to less interference from the non-selected cube probes.

An algorithm was developed at INSA de Toulouse in France, to allow for interfacing between the SoP, with its required digital inputs, a spectrum analyzer, and a computer for the processing of data. Although the algorithm was developed, it was never tested. Testing of the algorithm would be necessary to develop maps of EMI and to compare the findings with the results from the scan tables under conventional scanning.

Finally, the cube probe SoP concept can be used potentially as a means to inject noise into a circuit for aggression purposes and further EMC testing. 


\section{References}

[1] R. R. Tummala, "Moore's Law meets its Match", IEEE Spectrum, June 2006

[2] S. Ben Dhia, M. Ramdani, E. Sicard, "Electromagnetic Compatibility of Integrated Circuits - Techniques fow low emission and susceptibility", Springer, ISBN 0-387-26600-3, 2006

[3] A. Boyer, 2007,"Méthode de Prédiction de la Compatibilité Electromagnétique des Systèmes en Bôitier", PhD Thesis, Institut des Sciences Appliquées de Toulouse, France.

[4] J.J. Laurin, S.G. Zaky, K.G. Balmain, "EMI-Induced Failures in Crystal Oscillators", IEEE Transactions on Electromagnetic compatibility, Volume 33, Issue 4, Nov. 1991 Page(s):334 - 342

[5] M.P. Robinson, K. Fischer, I.D. Flintoft, A. C. Marvin, "A Simple Model of EMIInduced Timing Jitter in Digital Circuits, its Stastical Distribution and its Effect on Circuit Performance", IEEE Transactions on Electromagnetic Compatibility Volume 45, Issue 3, Aug. 2003, Page(s): $513-519$

[6] E. Sicard, A. Boyer, A. Tankielun, "On the Prediction of Near-field Microcontroller Emission", 2005 International Symposium on EMC, 3, 8-12 August 2005, Page(s): $695-699$

[7] A. Boyer, L. Roy, E. Sicard, B. Tamer," Cube Probe Structures for an Integrated Near Field Scanner Module", Electronics Letters, Volume 44, Issue 11, May 22, 2008, Page(s): 667 - 669

[8] A. Boyer, S. Bendhia, E. Sicard," Characterisation of Electromagnetic Susceptibility of Integrated Circuits using Near-field Scan", Electronics Letters, Volume 43, Issue 1, Jan. 4 2007, Page(s): $15-16$

[9] K.P. Slattery, J. Neal, W. Cui, "Near-field Measurements of VLSI devices", IEEE Transactions on EMC, Volume 41, Issue 4, 1999, Page(s): 374 - 384 [10] U. Inan, A. Inan, " Engineering Electromagnetics", Menlo Park, California, Addison Wesley Longman, Inc., 1999

[11] J. S. Dahele, A. L. Cullen, "Electric Probe Measurements on Microstrip", IEEE Transactions on Microwave Theory and Techniques, Volume 28, Issue 7, July 1980, Page(s): $752-755$ 
References

[12] S. Burkhart, "Coaxial E-Field Probe for Microwave Measurement (Short Papers)", IEEE Transactions on Microwave Theory and Techniques, Volume 33, Issue 3, March 1985, Page(s): $262-265$

[13] J. Krupka, C. Weil, "Recent Advances in Metrology for the Electromagnetic Characterization of Materials at Microwave Frequencies", $12^{\text {th }}$ International Conference on Microwaves and Radar, Volume 4, May 1998, Page(s): 243 253

[14] D. Popovic, M. Okoniewski, "Precision open-ended coaxial probe for dielectric spectroscopy of breast tissue", IEEE Antennas and Propagation Society International Symposium, Volume 1, June 2002, Page(s): $815-818$

[15] T. Michiyama, E. Tanabe, Y. Nikawa, "Obliquely Cut Open-Ended Coaxial Probe for Obtaining Complex Permittivity of Lossy Materials", Asia-Pacific Microwave Conference APMC 2006, December 2006, Page(s): 587 - 590

[16] M. Kanda, "Standard Probes for Electromagnetic Field Measurements", IEEE Transactions on Antennas and Propagation, Volume 41, Issue 10, October 1993

[17] M. Kanda, "An Electromagnetic Near-Field Sensor for simultaneous Electric and Magnetic Field Measurements", IEEE Transactions on Electromagnetic Compatibility, August 1984, Page(s): $102-110$

[18] M.E.G. Upton, A.C. Marvin," Improvements to an electromagnetic near-field sensor for simultaneous electric and magnetic field measurement", IEEE Transactions on Electromagnetic compatibility, Volume 35, Issue 1, February 1993, Page(s): 96-98

[19] A. Tran, S. McGarry, S. Nicol, J. Wojcik, M.C.E. Yagoub, "Design of Highly Sensitive H-Field Probes for Near-Field EMC \& HAC Assessment", IEEE ANTEM 2006 - 12th International Symposium on Antenna Technology and Applied Electromagnetics, July 2006.

[20] Y. Gao, I. Wolff, " A new miniature magnetic field probe for measuring threedimensional fields in planar high-frequency circuits" IEEE Transactions on Microwave Theory and Techniques, Volume 44, Issue 6, June 1996

[21] N. Ando, N. Masuda, N. Tarnaki, "Miniaturized Thin-film Magnetic Field Probe with High Spatial Resolution for LSI Chip Measurement", IEEE International Symposium on Electromagnetic Compatibility, August 2004, Page(s): $357-362$ 
References

[22] F. Cecelja, W. Balachandran," Optimized CdTe Sensors for Measurement of Electric and Magnetic Fields in the Near-Field Region", IEEE Transactions on Instrumentation and Measurement, Volume 49, Issue 3, June 2000

[23] M. Iwanami, E. Yamazaki, K. Nakano," Magnetic Near-Field Measurements over LSI Package Pins by Fiber-Edge Magnetooptic Probe", Journal of LightWave Technology, Volume 21, Issue 12, December 2003

[24] E. Suzuki, S. Arakawa, H. Ota, K. I. Arai, R. Sato," Optical Magnetic NearField Probe Working up to $15 \mathrm{GHz}$ Using CdTe Electrooptic Crystals", IEEE Transactions on Electromagnetic Compatibility, Volume 47, Issue 2, May 2005

[25] E. Suzuki, S. Arakawa, H. Ota, K. I. Arai, R. Sato," EO Probe for Simultaneous Electric and Magnetic Near-Field Measurements Using LiNbO3 with Inverted Domain", IEEE Transactions on Microwave Theory and Techniques, Volume 53, Issue 2, February 2005

[26] ANSI/IPC-6016: "Qualification and Performance Specification for High Density Interconnect (HDI) Layers or Boards", Association Connecting Electronics Industries, May 1999

[27] L. Lecheminoux, N. Gosselin," Advanced Design, Technology and Manufacturing for High Volume and Low Cost Production", International Electronics Manufacturing Technology Symposium IEMT 2003, July 2003, Page(s): $255-260$

[28] S. Vasudevan, A. Shaikh, "Microwave characterization of low temperature cofired ceramic system", $3^{\text {rd }}$ International Symposium on Advanced Packaging Materials, March 1997, Page(s): $152-157$

[29] Wu Ke-Li, Huang Yong, "LTCC technology and its application in high frequency front end modules", $6^{\text {th }}$ International Symposium on Antennas, Propagation and EM Theory, November 2003, Page(s): 730 - 734

[30] L. Roy, N. Berthereau, N. Hojjat, K. Kautio, H. Panesar, "A new dielectric resonator antenna with microstrip-waveguide transition in LTCC", IEE Electronics Letters, Vol. 42, No. 19, Sept. 2006.

[31] G. Brzezina, L. Roy, L. MacEachern, "Planar Antennas in LTCC Technology with Transceiver Integration Capability for Ultra-Wideband Applications", IEEE Transactions on Microwave Theory and Techniques, Volume 54, Issue 6, June 2006, Page(s): 2830 - 2839 
References

116

[32] G. Brzezina, L. Roy, L. MacEachern, "A miniature LTCC bandpass filter using novel resonators for GPS applications", European Microwave Comference, October 2007, Page(s): $536-539$

[33] G. Brzezina, L. Roy, "A miniature lumped element LTCC bandpass filter with high stopband attenuation for GPS applications", IEEE MTT-S International Microwave Digest Symposium, June 2008, Page(s): 1218-1215

[34] A. Shamim, M. Arsalan, L. Roy, M. Shams, G. Tarr, "Wireless Dosimeter: System-on-Chip Versus System-in-Package for Biomedical and Space Applications," IEEE Transactions on Circuits and Systems II: Express Briefs, Volume 55, Issue 7, July 2008, Page(s): $643-647$

[35] VTT Electronics, more information available at www.vtt.fi

[36] A. Shamim, L. Roy, N. Fong, N.G. Tarr, V. Levenets," On-Chip Antenna Measurements: Calibration and De-embedding Considerations", Proceedings of the IEEE Instrumentation and Measurement Technology Conference IMTC, 2005, May $16-19$, Page(s): $463-466$

[37] A. Shamim, J. Bray, L. Roy, N. Hojjat, R. Abou Elasoued, and D. Baillargeat, "Microwave and Magnetostatic Characterization of Ferrite LTCC for Tunable and Reconfigurable SiP Applications", IEEE MTT-S, Hawaii USA, June 2007, Page(s):. 691 - 694

[38] R. R. Tummala, M. Swaminathan, M.M. Tentzeris, J. Laskar, Chang GeeKung, S. Sitaraman, D. Keezer, D. Guidotti, Huang Zhaoran, Lim Kyutae, Wan Lixi, S.K. Bhattacharya, V. Sundaram, Liu Fuhan, P.M. Raj, "The SOP for miniaturized, mixed-signal computing, communication, and consumer systems of the next decade", IEEE Transactions on Advanced Packaging, Volume 27, Issue 2, May 2004, Page(s) $250-267$

[39] V. Sundaram, R.R. Tummala, G. White, K. Lim, Wan Lixi, D. Guidotti, Liu Fahan, S. Bhattacharya, R. M. Pulugurtha, I.R. Abothu, R. Doraiswami, R.V. Pucha, J. Laskar, M. Tentzeris, G.K. Chang, M. Swaminathan, "System-on-apackage (SOP) substrate and module with digital, RF and optical integration", Proceedings of the $54^{\text {th }}$ Electronic Components and Technology Conference, Volume 1, June 2004, Page(s): $17-23$

[40] S. K. Lim, "Physical design for 3D system on package", IEEE Design and Test of Computers, Volume 22, Issue 5, November 2005, Page(s): 532 - 539 
References

[41] W. Yun, V. Sundaram, M. Swaminathan," High-Q Embedded Passives on Large Panel Multilayer Liquid Crystalline Polymer-Based Substrate", IEEE

Transactions on Advanced Packaging, Volume 30, Issue 3, August 2007,

Page(s): $580-591$

[42] F. Yuan, "Fully differential 8-to-1 current-mode multiplexer for $10 \mathrm{Gbit} / \mathrm{s}$ serial links in $0.18 \mu \mathrm{m}$ CMOS", Electronics Letters, Volume 40, Issue 13, June 2004, Page(s): $789-790$

[43] D. Kehrer, H.-D. Wohlmuth, C. Kienmayer, A.L. Scholtz, "A 1 V monolithic transformer-coupled 30-Gb/s 2:1 multiplexer in $120 \mathrm{~nm}$ CMOS", IEEE MTT-S International Microwave Symposium Digest, Volume 3, June 2003, Page(s): $2261-2264$

[44] R. Jacob Baker, CMOS: Circuit Design, Layout, and Simulation, Second Edition. Hoboken, NJ: John Wiley \& Sons Inc., 2005, Page(s): $377-380$ 